\title{
ANNUAL REPORT AND SUMMARIES OF FY 1991 ACTIVITIES
}

\section{DIVISION OF ENERGY BIOSCIENCES}

SEPTEMBER 1991

U.S. DEPARTMENT OF ENERGY OFFICE OF ENERGY RESEARCH OFFICE OF BASIC ENERGY SCIENCES DIVISION OF ENERGY BIOSCIENCES WASHINGTON, D.C. 20585 


\section{Program Overview of the Division of Energy Biosciences}

At a time when the issues of energy resources for the future, environmental quality, and U.S. competitiveness in high technology markets are all high on the list of national priorities, research that is oriented towards contributing to the long-term solutions of these concerns is generally recognized to occupy a significant role. This is one of the conclusions of the National Energy Strategy document released in 1990. As a component of the Department of Energy, the Energy Biosciences (EB) program of the Office of Basic Energy Sciences suppots long-term research aimed at addressing energy-related problems utilizing biological systems.

The EB program generates critical fundamental biological data, principally about plants and microorganisms, that contribute toward establishing the bases for future biotechnologies related to energy matters. The studies supported by EB are broadranging, yet focus on understanding basic principles and mechanisms rather than on the optimization of processes, which is in the realm of other Federal programs and industry. However, one important facet of the program is to encourage interactions with, and transfer of information to, commercial interests and more applied research programs.

The ability of biological systems to perform myriads of chemical transformations resulting in constituents with unique properties has become progressively more evident in recent years. Over history, humankind has continued to exploit and modify biological systems for advantage. In recent years, enormous frontiers have been opened for utilization of biological processes as "biotechnologies" through the acquisition of crucial fundamental knowledge about biological systems. This has been facilitated particularly by discoveries on manipulating biosystems at the genetic level in ways previously unimagined. The primary challenge is to use the current and forthcoming information in a creative and prudent fashion in devising new technologies that will serve the needs of society without imposing undue risks to the health of its people and the natural environment which has suffered so seriously from some of man's other endeavors.

The EB program is oriented towards important research areas that are, in some cases, underpopulated and not well defined in either an intellectual framework or in potential approaches to the problem area. The program seeks projects that attempt to break new ground in providing new insights into the basic mechanisms of how plants and nonmedical microorganisms grow, survive, metabolize, differentiate, inherit traits and reproduce. This type of knowledge is critically important to fully integrate biology into the building of future technologies (biotechnology) aimed at serving society in an energy context. 
The EB program content is broad in scope and may be broken down into the three categories cited below. Despite the divisions noted below, it is clear that the topics must and do integrate from the standpoint of natural processes, as well as in the formulation of approaches and insights in the research supported. The components of the EB program consist of:

I. Primary Biological Energy Conversion. This category comprises research on plant and microbial photosynthesis, the solar energy driven process central to the support of life on earth. This category includes studies on the initial carbon dioxide fixing mechanisms as well as the associated water splitting and other component reactions. The program also supports research on the fundamental processes, such as the control of growth and development, that ultimately govern the form and amount of biomass a plant produces. In addition, investigations on plant interactions with the natural environment (including stress reactions, as well as the interactions with biological agents such as pathogens) are included in this category. Constituents of this broad research category include biophysical, biochemical and physiological, and genetic investigations.

II. Bioconversion of Products. The utilization of the products of the primany energy conversion process is the nature of this second category. A prime component is studies on the diverse metabolic capabilities of plarts and microorganisms in synthesizing materials that may ultimately be utilized for fuels and chemicals. These fundamental investigations focus on understanding pathways of metabolism and the genetic and biochemical regulatory mechanisms that determine the nature and amount of metabolic compounds converted. Of special interest are the synthesis, structure and function of plant cell walls, the predominant renewable biomass resource. Also of interest are the mechanisms by which lignocellulose of plant cell walls is degraded biologically into compounds of potential utility. Associated with this category is how plant-microbial interactions occur in symbiotic energy exchanges. A substantial amount of this category covers fundamental microbial conversions, such as methanogenesis and other diverse fermentative pathways.

III. Infrastructure. Underpinning of the developmert of future biotechnologies is a key objective of the EB program. In this category are efforts to better understand genetic mechanisms involving the transfer of genetic information and its expression in plant and microbial species that oftentimes have not been studied very extensively. Also under study are genetic mechanisms in better known species such as maize where certain phenomena were first discovered. Further, in this portion of the program are such activities as the development of critical data bases, specific techniques and instrumentation. A training component for nurturing areas that are important but underpopulated is also encompassed. 
Representative of some of the activities sponsored by EB are the following:

\section{Mutant Analysis of Photosystem I of Photosynthesis}

If a process is to be analyzed genetically, by use of mutants in which that process is altered or defective, it often helps to find conditions in which the process is not needed. The photosynthetic activity of plants and cyanobacteria (once called blue-green algae) is dependent upon the activity of two light-utilizing systems, known as photosystem I and photosystem II. Using recombinant DNA techniques and the genetically manipulable unicellular cyanobacterium, Synechocystis, which can be grown without photosystem II, investigators succeeded several years ago in clarifying the nature of the electron donor to photosystem II. However, because the activity of photosystem I is essential for the growth of every photosynthetic organism that could be easily manipulated genetically, genetic analysis of photosystem I had progressed very little. That situation has now changed with the progress made by two groups at the Michigan State University Plant Research Laboratory (PRL). It has been shown that Synechocystis may be grown without photosynthesis if it be given only five minutes of photosynthetically inactive blue light per day; the group was thereupon able to mutagenize a polypeptide at the very core of photosystem I. Another group, in collaboration with researchers from Germany and Spain, showed that a strain of the filamentous cyanobacterium, Anabaena, that can grow in complete darkness, can also be mutated in chosen genes by the use of recombinant DNA techniques and transfer of altered DNA from Escherichia coli to Anabaena. These findings are enabling researchers in the PRL and elsewhere to define photosystem I with a more detailed analysis and thus enhance the overall understanding of the structure and function of the photosynthetic apparatus.

\section{Mechanisms of Plant/Fungal Pathogen Interactions}

Fungal toxins that are highly specific to a particular host plant have proven agriculturally devastating, for example, in the Southern Corn Leaf Blight epidemic of 1970. Although several such toxins were known, the biosynthetic apparatus responsible for their synthesis has been elusive. Moreover, in no instance was it precisely known why closely related strains of the same host plant were resistant to the fungal toxin. Both of these questions have recently been broached mainly through the efforts of researchers at the PRL. First, by combining molecular biology with biochemistry, it has been possible to isolate the genes responsible for the biosynthesis of the host-specific toxin produced by a strain of Cochilobolus (earlier called Helminthosporium). Second, experiments have demonstrated that only strains of corn that are resistant to the toxin express an enzymatic activity, "HCtoxin reductase", that can metabolize the toxin. In collaboration with an investigator at Pioneer Hi-Bred International Inc., it was shown that the plant gene that controls resistance or sensitivity to the fungus also controls the activity of this enzyme. These studies are leading to the first complete, genetic, molecular, and biochemical understanding of a plant disease interaction, where toxins are involved. 
Another event related to EB supported research occurred when the U.S. Patenit Office celebrated the awarding of its five millionth patent on March 19, 1991. The patent for work on the transference of genes for combining the metabolism of five carbon sugars with alcohol production in bacteria was awarded to the University of Florida and Dr. Lonnie Ingram, the principal investigator, and colleagues. The work was based on fundamental research supported by the EB program on the control of metabolic fluxes in bacteria, with subsequent USDA Competitive Research Grant support of the more applied aspects. The microorganisms are now in the hands of a biotechnology firm for the development of a commercial process, reflecting the transfer of basic information to potential applications and, finally, commercial products.

\section{Other Items Relating to the EB Program}

The institutions included in the joint USDA/DOE/NSF Plant Science Center program, begun in 1988, are being reviewed as originally planned by the involved agencies. The intent is to ascertain after roughly three years of operation how well the various centers have progressed in meeting their original goals. Each of the reviews involves use of scientific peers as well as representatives of the participating agencies. No decision has yet been made about the continuance of the plant science centers program beyond the originally scheduled five years.

The fiscal year (FY) 1991 activities of the EB program were many and varied. Following receipt of a record number of research applications in FY 1990, a new application process was implemented to try to diminish the mainr investment of time and energy by research applicants and the scientific reviewers of the applications. In FY 1991, potential applicants were requested to submit brief preapplications prior to any formal proposal submission. The pre applications were screened for topic areas that were outside the scope of the program and those applicants were discouraged from submitting proposals. Because of the extreme tightness of the funds initially in FY 1991, ideas which were at the time underrepresented in the EB program were encouraged for submission; others were not. The EB program also implemented, on an experimental basis, a reduction of proposal length from a 15 page to a 10 pag's limit, again to try to diminish workloads of both applicants and reviewers.

In FY 1991 the EB program began sponsorship of thiree more Life Sciences Research Foundation (LSRF) postdoctoral fellowships. These competitive fellowships represent on the part of EB a coverage of plant and microbial sciences that other LSRF sponsors do not support. This year's fellows, all working in the plant sciences (Douglas Cook at the Carnegie Institution of Washington; Zhongchi Liu at the California Institute of Technology; Jason Reed at The Salk Institute), join the two previous fellows who are investigating micrnbial systems. 
The breakdown of how the resources available to EB were distributed in FY 1991 is indicated in the following table.

\begin{tabular}{|c|c|}
\hline $\begin{array}{l}\text { Number } \\
\text { of } \\
\text { Projects }\end{array}$ & $\begin{array}{c}\text { FY } 91 \\
\text { funding } \\
\text { (in thousands) }\end{array}$ \\
\hline
\end{tabular}

\begin{tabular}{|c|c|c|c|}
\hline $\begin{array}{l}\text { University and Non-Profit } \\
\text { Institutions }\end{array}$ & 157 & $\$ 14,662$ & $66 \%$ \\
\hline Michigan State University & & & \\
\hline $\begin{array}{l}\text { Plant Science Centers } \\
\text { Universities }\end{array}$ & 2 & 1,296 & $6 \%$ \\
\hline $\begin{array}{l}\text { National Laboratories } \\
\text { Brookhaven National Lab } \\
\text { Lawrence Berkeley Lab } \\
\text { Los Alamos National Lab }\end{array}$ & 11 & 2,179 & $10 \%$ \\
\hline National Renewable Energy Lab." & 1 & 125 & $1 \%$ \\
\hline $\begin{array}{l}\text { Small Business Innovation } \\
\text { Research (SBIR) contribution, } \\
\text { and Miscellaneous }\end{array}$ & & 680 & $3 \%$ \\
\hline $\begin{array}{l}\text { Conferences, Educational } \\
\text { Activities }\end{array}$ & 9 & 349 & $1.5 \%$ \\
\hline Databases & 1 & 75 & $0.5 \%$ \\
\hline & 195 & 2,075 & \\
\hline
\end{tabular}

The total funding figure is a net figure after a Congressionally mandated general reduction.

*formerly known as the Solar Energy Research Institute 
The EB program also provided partial funding for the following scientific meetings and other activities for the transfer of information:

1. Summer Investigations into the Metabolic Diversity of the Microbial World, Marine Biological Laboratory, Woods Hole, MA, Summer 1991.

2. Symposium on Ubiquitin and Intracellular Proteolytic Degradation, Federation of American Societies for Experimental Biology, Saxtons River, VT, June 9-14, 1991.

3. VII International Symposium on Photosynthetic Prokaryote?s, University of Massachusetts, Amherst, MA, July 23-27, 1991.

4. Research Conference on The Pyrroles of Photosynthetic Organisms, University of California, Davis, August 4-9, 1991.

5. Fifth International Fungal Spore Conference, Unicoi State Park, GA, August 17-21, 1991.

6. Cold Spring Harbor Conference on the Molecular Biology of Signal Transduction in Plants, Cold Spring Harbor Laboratory, Cold Spring Harbor, NY, October 2-6, 1991.

7. Photosynthetic Responses to the Environment, University of Hawaii, Kona, HI, August 25-27, 1992.

As stated in previous annual reports the EB staff wishes to express its sincere appreciation to those members of the scientific community who have donated their time and efforts in performing evaluations for the program. Once again it needs to be said that quality assurance and maintenance of any program can only be achieved by rigorous and critical evaluation activities offered as a donation by the research community. This cooperation and generosity by scientists in this country and abroad is gratefully acknowledged.

Lastly the staff of EB wishes again to recognize the contributions of Dr. George E. Stapleton to the early planning and initiation of the EB program (originally called the Biological Energy Research program). Recently, Dr. Stapleton died suddenly after years of service as a microbiologist and radiation biologist at the Oak Ridge National Laboratory, the Atomic Energy Commission, the Energy Research and Development Administration and the Department of Energy. 
Anyone wishing more information about the Energy Biosciences program is invited to write or call:

Dr. Robert Rabson or

Dr. Gregory L. Dilworth

Division of Energy Biosciences

ER-17, GTN

U.S. Department of Energy

Washington, D.C. 20585
Voice (301) $903-2873$

Fax (301)903-6594

PLEASE NOTE: BEFORE NOVEMBER 9, 1991, THE

NUMBERS ARE

Voice (301) 353-2873

Fax (301) $353-6594$

Office Staff: Patricia Snyder and Mary Jo Martin 


\section{U.S. Department of Agriculture Madison, Wisconsin 53705-2398}

\section{Molecular Organization in the Native State of Woody Tissue: Studies of Tertiary Structure Using the Raman Microprobe, Solid State ${ }^{13} \mathrm{C}$ NMR and Biomimetic Tertiary Aggregates \\ R.H. Atalla, Forest Products Laboratory \\ $\$ 165,000$ (FY 90 Funds/2 years)}

Studies of wood cell wall structure using the Raman Microprobe have revealed significant variations in the molecular architecture of cell walls. These observations have raised questions about the relationship between molecular and morphological levels of organization and about the intermolecular interactions which underlie such organization. Our objectives in this program are: (1) to achieve further definition cf the variation of molecular organization; (2) to explore the intermolecular interactions which determine the patterns of aggregation of the constituents of the cell walls. We have confirmed and extended the results of our earlier studies based on Raman Microprobe spectroscopy. The Raman based investigations have been complemented by more limited studies utilizing Solid State ${ }^{13} \mathrm{C}$ NMR. The exploration of intermolecular interactions which dominate the organization of the cell walls is currently centered on the development of biomimetic tertiary aggregates which simulate the states of aggregation which prevail in the cell walls. These are prepared by culturing cellulose producing bacteria in media which contain analogs of the other cell wall constituents. We have confirmed that analogs of hemicelluloses can alter the aggregation of the cellulose to make it more similar to higher plant celluloses. These results suggest that one of the functions of the hemicelluloses is the regulation of the aggregation of cellulose. Lignin precursors also have an effect on the aggregation of cellulose but it is more subtle. Our spectroscopic studies have revealed evidence of charge transfer complexes between lignin precursors and oligosaccharides. These observations require reassessment of the current models of lignin biogenesis and function. We also continue to explore the pathways available for charge transport within the structures we are investigating, both native and biomimetic.

\section{U.S. Department of Agriculture Raleigh, North Carolina 27695-7631}

2. Control of Sucrose Biosynthesis in Plants by Protein Phosphorylation S.C. Huber, USDA/ARS and Departments of Crop Science and Botany, NCSU

$$
\$ 120,000 \text { (2 years) }
$$

Sucrose-phosphate synthase (SPS) is a widely acknowledged key to the regulation of sucrose allocation iri plants, yet much remains to be learned about the biochemical mechanisms which regulate its activity. In leaves, SPS activity is known to be controlled by covalent modification and there is increasing evidence that protein phosphorylation is the mechanism. When spinach leaves are illuminated, SPS is activated/dephosphorylated (by an okadaic acid-sensitive SPS protein phosphatase), and upon transfer to darkness, SPS is inactivated/phosphorylated (SPS kinase). These reactions can be demonstrated both in vivo and in vitro. Our recent findings suggest that SPS is phosphorylated at multiple sites, only some of which are of direct regulatory significance. The phosphorylation of at least two sites is involved in the inactivation that occurs upon darkening of leaves. Other sites appear to be "constitutively" phosphorylated, but when dephosphorylated, result in inactivation of SPS. Thus, phosphorylation of this enzyme is complex. We now propose to: 1) sequence the phosphorylation sites, and determine whether the sites are "clustered"; 2) purify and 
characterize the protein kinase and protein phosphatase; and 3) investigate the mechanisms and "signals" which control the phosphorylation status of SPS in vivo iwith focus on metabolites and possible "coarse control" of the protein kinase and protein phosphatase). Overall, the projected studies will help establish the new and important concept that carbohydrate metabolism (specifically sucrose biosynthesis) in plants is controlled by protein phosphorylation.

\section{Arizona State University Tempe, AZ 85287-1604}

\section{Antenna Organization in Green Photosynthetic Bacteria R.E. Blankenship, Department of Chemistry}

$\$ 219,054$ (2 years)

All photosynthetic organisms contain chlorophyll pigments that function as an antenna, absorbing light and transferring excitations to a photochemical reaction center where energy storage takes place by a series of chemical reactions. The green photosynthetic bacteria are characterized by large antenna complexes known as chlorosomes. The overall objective of this project is to determine the molecular organization and mechanism of excitation transfer in chlorosome antennas. The chlorosome pigments are organized in vivo into pigment oligomers in which direct pigment-pigment interactions are of dominant importance. Time-resolved fluorescence spectroscopy has been used to identify the pathway and kinetics of excitation flow from the peripheral region of the chlorosome, to the site where the chlorosome attaches to the membrane, into the membrane-found antenna complexes and finally into the reaction center. Recent work has identified a redox-activated control of energy transfer efficiency in green sulfur bacteria. At high redox potential, most excitations are quenched within the chlorosome, while at low redox potentials almost all are transferred to the reaction center. The quenching effect may be a control mechanism that protects the cell during conditions where light and oxygen are present simultaneously. Some current work is directed toward identification of the mechanism of this redox-activated control effect. In addition, model compounds are being used to gain more information about the structure of the pigment oligomers found in chlorosomes.

\section{Arizona State University} Tempe, AZ 85287-1604

\section{Center for the Study of Early Events in Photosynthesis}

R.E. Blankenship, J.P. Allen, W.D. Frasch, J.D. Gust, S.H. Lin, A.L. Moore, T.A. Moore, G.R. Seely, W.F.J. Vermaas, A.N. Webber and N.W. Woodbury $\$ 1,295,882$ (30 months)

A USDA/DOE/NSF Plant Science Center for the Study of Early Events in Photosynthesis was established at Arizona State University in 1988. This Center serves as an infrastructure supporting individual ASU scientists who study photosynthesis using a wide range of different methods and approaches, ranging from molecular biology and biochemistry to organic chemistry, ultrafast laser spectroscopy, X-ray crystallography and theoretical chemistry. The Center is structured to foster multidisciplinary cooperative research projects. In addition, the Center brings visiting scientists to ASU. Graduate and postdoctoral training programs are central components of the activities of the Center.

The ultimate objective of the research that is carried out at the Center is to elucidate the basic principles that govern the biochemical and biophysical processes of photosynthetic energy storage. This goal is being sought via investigation of the early events of photosynthesis, including: light absorption and excitation transfer in photosynthetic antennas; the mechanism of primary 
photochemistry in plant and bacterial systems; secondary electron transfer processes; structure and assembly of photosynthetic antennas reaction centers and electron transfer proteins; pigment-protein interactions; artificial and biomimetic photosynthetic systems; and mechanisms of biological electron transfer reactions.

*(A unit of the USDA-DOE-NSF Plant Science Centers program.)

\section{Arizona State University Tempe, AZ 85287-1601}

\section{Specific Mutagenesis of a Chlorophyll-Binding Protein W.F.J. Vermaas, Department of Botany}

Chlorophyll, absorbing light energy for use in photosynthesis, is bound by specific proteins in thylakoid membranes. The exact nature of chlorophyll-protein interactions and the identity of protein residues involved in chlorophyll binding are still largely unknown. In a number of chlorophyll-binding proteins, including the photosystem II protein CP47, several pairs of His residues spaced by 12-15 other amino acid residues are found in hydrophobic regions. These His residues have been suggested to serve as chlorophyll ligands. To test the necessity of these His residues in CP47, specific His residues have been altered to Tyr or Asn, and the effects of these mutations are now studied in vivo in the cyanobacterium Synechocystis sp. PCC 6803. Most mutations lead to a decreased photoautotrophic growth rate of the cyanobacterium, due in part to a decreased stability of the photosystem II complex. The effect of multiple His mutations introduced simultaneously appears to approximate the sum of the effects of the individual mutations. The effective relative antenna size of photos'ysiem II in the various CP 47 mutants is now being measured.

The CP47 protein may also be involved in events at the donor side of photosystem II. In particular, a large hydrophilic domain in the protein has been suggested to play a role in constituting the watersplitting complex. We have now made deletions of 9-15 residues in the hydrophilic regions of CP47, and we have identified a region which can be deleted without obvious ill effects, and a domain which appears crucial for photosystem II structure and function. Residues of particular importance will be defined by further mutagenesis experiments.

\section{University of Arizona Tucson, AZ 85721}

\section{Role of Pectolytic Enzymes in the Programmed Release of Cells from the Root Cap of Higher Plants \\ M.C. Hawes, Departments of Plant Pathology and Molecular \& Cellular Biology}

$\$ 170,500$ (2 years)

Many plant species releace thousands of healthy single root "border" cells from their root tips daily. This controlled release of living somatic cells into the environment is unique among higher organisms, and its function is unknown. The purpose of this project is to elucidate the biochemical and molecular mechanism(s) of border cell separation. Pisum sativum was used as a model to demonstrate that border cell separation is developmentally regulated, and can by synchronized experimentally. Synchronized root tips of pea have been used to test the hypothesis that border cell separation is associated with the activity of pectolytic enzymes capable of degrading pectin-rich substances of the middle lamella. Activity of two different types of pectolytic enzymes - polygalacturonase and pectinmethylesterase - has been detected and shown to be correlated with the programmed separation of border cells. The goals of the project are to determine the kinds and number of pectolytic enzymes 
whose activity is induced in concert with border cell separation; to clone the genes encoding the enzymes; and to test the role of the genes in cell separation. Hairy roots induced by inoculation of pea seedlings with Agrobacterium rhizogenes will be used to study expression of genes encoding pectolytic enzymes, and to analyze their role in border cell separation.

\section{University of Arizona \\ Tucson, AZ 85721}

\section{Phytoalexin Detoxifying Enzymes in the Plant Pathogenic Fungus Nectria Haematococca} H.D. VanEtten, Department cf Plant Pathology

$\$ 154,501$ (2 years)

The production of antimicrobial compounds (phytoalexins) by plants is believed to function as an active mechanism for disease resistance. Previous work in our laboratory has indicated that plant pathogenic fungi can circumvent a phytoalexin-based plant resistance mechanism by detoxifying their host's phytoalexins. Our primary model has been the diseases of pea and chickpea caused by the fungus Nectria haematococca. Conventional genetic, molecular genetic, and biochemical approaches have demonstrated that detoxification of the pea phytoalexin pisatin via a substrate inducible cytochrome P-450 monooxygenase is required for pathogenicity on pea. Detoxification of the phytoalexins from chickpea, medicarpin and maackiain, is also catalyzed by monooxygenases. While the monooxygenases in Nectria are encoded by a gene family, only certain members of the family are associated with pathogenicity on pea or chickpea. We propose to use sequence analysis, chimeric gene construction, and in vitro mutagenesis to identify the primary structural features in these cytochrome P-450 proteins that are responsible for their specific biochemical and biological properties. A comparison of these genes with other cytochrome P-450 genes should reveal whether common features of the genes are associated with pathogenicity and might reveal how these genes evolved.

\section{Boston College Chestnut Hill, MA 02167}

\section{Osmoregulation in Methanogenic Archaebacteria M.F. Roberts, Department of Chemistry}

$\$ 166,000$ (2 years)

Nuclear Magnetic Resonance techniques are used to investigate osmoregulation in methanogenic archaebacteria. Experiments fall into 3 basic groups: (1) in vivo NMR studies of Methanococcus thermolithotrophicus, Methanogenium cariaci, and Methanosarcina barkeri using soluble substrates $\left(\mathrm{H}^{13} \mathrm{COONa} \mathrm{Na}^{+},{ }^{13} \mathrm{CH}_{3} \mathrm{OH},{ }^{13} \mathrm{CH}_{3}{ }^{13} \mathrm{COO}^{-} \mathrm{Na}^{+}\right.$, or their nonenriched counterparts) for methanogenesis to monitor osmolyte production, uptake, or loss; (2) use of ${ }^{13} \mathrm{C}$ - and ${ }^{15} \mathrm{~N}$-labeled potential precursors and in vitro analyses of specific label uptake for elucidation of biosynthetic pathways of the B-amino analogues which serve as osmolytes in these organisms (including B-glutamate and $\mathrm{N}_{\Sigma}$-acetyl-Blysine), and isolation of key biosynthetic enzymes (e.g., lysine 2,3-aminomutase); and (3) identification (relying on 2D NMR techniques) of organic solutes in other methanogers which appear in response to non-permeant electrolytes and nonelectrolytes. This latter will be done in collaboration with several other groups working on methanogens. The ultimate goal in understanding how these anaerobic organisms deal with osmotic stress is to provide insights into increasing the salt tolerance of other cells. 


\title{
Boyce Thompson Institute for Plant Research, Inc. Ithaca, NY 14853
}

\author{
9. Differential Regulation of Plastid mRNA Stability \\ D.B. Stern
}

$\$ 147,990$ (FY 90 Funds/2 years)

The expression of photosynthetic proteins requires a cooperative interaction between the nuclear and plastid genomes, and the development of molecular tools to manipulate plants depends in part on understanding how these genes are regulated. Our research focuses on mRNA synthesis and the control of mRNA stability in higher plant chloroplasts, since mRNA accumulation is an important regulatory mechanism during plastid differentiation. Plastid mRNA accumulation is influenced by promoter activity, RNA secondary structure, RNA-binding proteins, and the activities of ribonucleases. An in vitro system has been developed from spinach chloroplasts that allows study of each of these factors. Thus far, we have found that a stem/loop structure located at the mRNA 3' end is required for RNA stability in vitro, and that the sequence of the loop as well as the stem can significantly affect RNA half-life. We have characterized and partially purified several RNA-binding proteins from spinach ihloroplasts. At least one of these proteins binds at the stem/loop in a base-specific manner, and appears to play roles in transcription termination and RNA processing. At least three ribonuclease activities have been characterized: one is a non-specific exoribonuclease that may be a scavenger enzyme required for nucleotide turnover, and two are endonucleases that cleave within or near the stem/loop and are potentially rate-limiting enzymes for mRNA decay in vivo. We are continuing to purify these nucleases and RNA-binding proteins, and have initiated work at the organellar level to characterize the associations of mRNA and RNA-binding proteins ir; vivo during plastid differentiation. One long-term goal is to test the results obtained in vitro using recently developed chloroplast transformation systems.

\section{Brandeis University Waltham, MA 02254}

\section{Carbon and Hydrogen Metabolism of Green Algae in Light and Dark} M. Gibbs, Department of Biology

$\$ 153,814$ (2 years)

The key to an elucidation of the oxyhydrogen reaction coupled to carbon dioxide reduction in the dark in the hydrogen-adapted green alga, Chlamydomonas reinhardtii is the source of ATP required to drive the reductive pentose phosphate cycle. The isolated intact darkened chloroplast evolves $\mathrm{CO}_{2}$ aerobically from specifically labeled glucose indicating the glycolytic and the oxidative pentose phosphate pathways with the latter dominating. Evolution of $\mathrm{CO}_{2}$ is blocked by mitochondrial inhibitors (amytal, rotenone, antimycin A, salicylhydroxamic acid, azide, propyl gallate) and is dependent upon oxygen indicating a chloroplastic respiratory pathway. Thylakoids in the dark and in the presence at DCMU oxidize reduced pyridine nucleotide (NADH and NADPH) coupled to the formation of adenosine triphosphate. The ratio of pyridine nucleotide oxidized to phosphate esterified can be regulated by the concentration of the pyridine nucleotide. The rate of adenosine triphosphate formed is sufficient to account for the observed rate of $\mathrm{CO}_{2}$ assimilated (3-4 $\mu$ mole per milligram chlorophyll per hour) by the intact algal cell. The oxyhydrogen reaction like $\mathrm{O}_{2}$-evolving photosynthesis and photoreduction $\left(\mathrm{H}_{2}, \mathrm{CO}_{2}\right.$, light) is a chloroplastic system. 


\title{
Brookhaven National Laboratory Upton, NY 11973
}

\author{
11. Plant Molecular Genetics \\ B. Burr and F.A. Burr, Biology Department
}

$\$ 315,000$

We have developed a means of rapidly mapping genes in maize using families of recombinant inbreds. These permanent populations permit many geneticists to contribute to the mapping effort and profit from each other's work at the same time. They have been used to compile a detailed molecular genetic map. These populations are ideal for mapping quantitative trait loci - those genes having metric rather than qualitative effects upon the traits iney control. Precise mapping of these genetic factors benefits from a densely populated map, and the use of replicated progeny improves the estimation of the genetic, compared with the environmental, component of variation. Two model traits are under study: anthocyanin pigmentation and telomere length both vary quantitatively ir, recombinant inbred populations.

Many of the structural and regulatory genes involved in pigment biosynthesis have been identified and cloned from maize. Preliminary analysis indicates that alleles of two regulatory genes are segregating in one population to account for $40 \%$ of the observed variation. We are attempting to see if there is a niolecular correlation at some level in the expression of these genes with the observed phenotype.

Telomeres, the chromosomal termini, are required for faithful chromosome replication and are themselves synthesized by a special set of enzymes. Telomere lengths vary more than 20 fold among maize inbreds surveyed. These lengths segregate reproducibly in a recombinant inbred family where $90 \%$ of the variation can be accounted for by eight loci. We are attempting to learn whether any of the segregating loci correspond to homologous genes identified in yeast that affect telomere replication.

\section{Brookhaven National Laboratory Upton, NY 11973}

12. Molecular Bases and Photobiological Consequences of Light Intensity Adaptation in
Dunaliella tertiolecta
P.G. Falkowski and J. LaRoche, Dept. of Applied Science

The long-term goal of this research effort is directed towards elucidating the molecular bases for changes in abundance and composition of pigment proteins in response to variations in irradiance. The primary organism under investigation is a unicellular marine chlorophyte, Dunaliella tertiolecta, which has a photosynthetic appara!us similar to that found in higher plants. The project aims at understanding how pigment synthesis and protein synthesis are coordinated and regulated so that functional pigment protein complexes are formed or degraded in response to light intensity. Attention is focused on level of control of light harvesting complex. Four genes which encode for light harvesting chlorophyll a/b proteins of photosystem II have been isolated and one cDNA clone has been sequenced. Northern blots of total RNA probed with the cab gene revealed a large increase in cab mRNA levels following a shift from high to low irradiance levels. Hybridization with other photosynthetic gene probes demonstrated that the response is specific to cab genes. The possibility that a shift from high to low light intensity stimulates the transcription of the cab genes is being studied with isolated nuclei using in vitro run off transcription assays. Several genomir clones, corresponding to the cDNA clones, are being characterized to identify upstream control regions. 


\title{
Brookhaven National Laboratory Upton, NY 11973
}

\author{
13. Regulation of Energy Conversion in Photosynthesis \\ G. Hind, Biology Department
}

$\$ 357,000$

Photosynthetic electron transport, energy transformation, and regulatory processes are studied by nondestructive biophysical techniques coupled with the isolation and characterization of membrane-associated enzymes and complexes. The pigment-protein complexes of thylakoids are organized into chiral macrodomains that presumably influence long range energy migration. Circular dichroism spectroscopy of mutant and wild-type thylakoids is used to monitor the reversible effects on macrodomain size of electrostatic screening, osmotic pressure of the medium, and energization of the membrane. Much, but not all of the organizational force resides in the light-harvesting chlorophyll $a / b$ complexes (LHC-II).

Slow structural adaptations known as state transitions also originate in LHC-11. They fine-tune the apportioning of excitation energy between the photosystems and are effected through activity of a membrane-bound kinase and a phosphatase. We have cloned and sequenced the gene for the protein kinase and have purified the phosphatase. The mechanism through which kinase activity is control'ed by ambient redox poise is unknown and is studied using biuchemical and genetic approaches. Redox poise also sets the relative activity of cyclic to non-cyclic electron flow, with cyclic becoming maximally active at low potentials. We are specially interested in low potential carriers, loosely associated with the stromal aspect of the thylakoid, that return electrons from photosystem 1 to plastoquinone. These include ferredoxin, NADPH:ferredoxin oxidoreductase and presumably a plastoquinol:ferredoxin oxidoreductase whose identity we seek.

This work will provide knowledge of mechanisms that optimize photosynthetic efficiency while possibly also protecting the thylakoid against photoinhibition and photodestruction under stress.

\section{Brookhaven National Laboratory Upton, NY 11973}

\section{The Physiology and Biochemistry of Cyanobacteria H.W. Siegelman, Biology Department}

$\$ 250,000$

The phages of cyanobacteria are being cloned, purified and characterized. Ten phage LPP-1 and LPP-2 strains were purified by $\mathrm{CsCl}$ gradient centrifugation and their morphology and mass are being determined by STEM. These phages are all short-tailed iscosohedral in morphology with a mass of about $50 \times 10^{6} \mathrm{Da}$. The phage DNA consists of about 40,000 base pairs. The LPP-1 and LPP-2 phages were originally classified by serological reaction. The two classes of phages can be categorized by their restriction fragment patterns, and each strain has a unique restriction fragment map which permits strain identification. PCR amplification of the full-length genomic phage DNA with a degenerate primer for the RNA polymerase gene yielded an oligonucleotide fragment of about 250 $\mathrm{bp}$. A primary objective is to determine the entire genomic DNA sequence of a cyanobacterial phage for comparison with the sequence of the T7 phage.

The energy collected by the phycobiliprotein antenna system is transferred to chlorophyll by allophycocyanin. The structural properties of allophycocyanin and its two subunits is being examined by $x$-ray scattering and fluorescence lifetime measurements. 


\section{Brown University \\ Providence, RI 02912}

\section{5. $\delta$-Aminolevulinate Biosynthesis in Oxygenic Prokaryotes \\ S. Beale, Division of Biology and Medicine $\quad \$ 191,000$ (2 years)}

Tetrapyrrole pigments function as essential components in the processes of respiration and photosynthesis, and also exist as cofactors in other important enzyme reactions. The tetrapyrroles arise from a branched biosynthetic pathway having $\delta$-aminolevulinic acid (ALA) as its first committed member. ALA is known to be formed by two distinct routes: by condensaticin ui yiycine and succinylCoA in animal, fungal, and some bacterial cells, and by transformation of the intact carbon skeleton of glutamate in plants, algae, and other bacterial ceiis. We are characterizing the reaction components for ALA biosynthesis derived from oxygenic prokaryotes, comparing them to their counterparts in plants, and studying the regulation of their activity in response to light and nutritional status. The potential of the prokaryotes for molecular genetic studies is being exploited by generating ALA auxotrophs, and identifying the enzymatic lesions by in vitro reaction complementation with purified, identified reaction components obtained from wild-type cells. Genetic complementation of the auxotrophic cells will be carried out by plasmid transformation with genomic libraries obtained from wild-type cells and carried in E. coli. The genes coding for the macromolecular reaction components will be isolated, identified, and made available for use as probes for studying the regulation of their expression during adaptation of the cells to light and nutritional status. The probes will also be evaluated for use in measuring expression of analogous genes in eukaryotic algae and higher plants.

\section{California Institute of Technology Pasadena, CA 91125}

\section{Genetics in Methylotrophic Bacteria M.E. Lidstrom, Environmental Engineering Science}

$\$ 179,999$ (FY 90 Funds/2 years)

The purpose of this project is to use genetic techniques to study the transcriptional regulation of C-1 specific functions in methylotrophic bacteria. The approach involves analyzing $\mathrm{C}-1$ specific genes in a facultative methanol utilizer, Methylobacterium extorquens AM1 and using this organism as a host to study genes encoding similar functions in methane-utilizers. We have focused on methanol oxidation genes, and have cloned several of these from both methanol and methane utilizing bacteria. We are now analyzing the promoter regions from some of these genes. We have cloned a fragment upstream of moxF from $M$. extorquens AM1 and from a Type I methanotroph, Methylomonas albus BG8 into a broad host range promoter cloning vehicle that uses lacZ as the reporter gene, and fragments show orientation-specific B-galactosidase activity, suggesting they contain promoter sequences. However, the promoter-containing fragment from $M$. extorquens $A M 1$ is constitutively expressed. We have shown that this improper regulation is an artifact of the plasmid construction, and chromosomal constructions are regulated properly. We have used this fusion in the chromosome to assay transcription of moxF in a series of Mox mutants that exhibit pleiotropic phenotypes. One of these, MoxB, exhibits only background transcription from this promoter, suggesting that moxB encodes a transcriptional regulator. Current work involves testing other putative regulatory mutants for defects in transcription, as well as defining the functions deficient in each. 


\title{
California Institute of Technology Pasadena, CA 91125
}

\author{
17. Molecular and Genetic Analysis of LEAFY, a Gene Controlling Floral Induction and \\ Flower Development in Arabidopsis thaliana \\ E. Meyerowitz, Division of Biology \\ $\$ 232,940$ (2 years)
}

Little is known of the biochemical and molecular mechanisms of plant growth and development. In particular, we are ignorant of the means by which cells in growing plants learn their position, and, as a consequence, differentiate to the cell type appropriate for that p.rsition in the plant. This is perhaps the central question in plant developmental biology, and a key question that must be answered before we can find new methods of controlling plant cellular differentiation in ways thict are useful. One proven approach to understanding the spatial control of differentiation in animals is the genetic approach: obtain mutations in which the process of interest is specifically disrupted, then study at the genetic and molecular levels the products and functions of the wild type copies of the genes that have been identified by these mutations. The goal of the project is to apply the genetic approach to an understanding of pattern formation in plants. Specifically, we will st' dy the Arabidopsis gene LEAFY, whose product is necessary for the vegetative to floral transition, and for the appropriate patterning of organs in flowers. Plants homozygous for mutant copies of this gene make vegetative rather than floral organs. We will begin with a series of genetic experirnents, to learn the exact role of the LEAFY product in meristem development. Simultaneously, the LEAFY gene will be cloned, and the sequence of its protein product determined. The cloning is to be done by chromosome walking from a nearby cloned RFLP marker. The chromosome walk is already almost complete. The sequence information obtained from the cloned gene will be used in further experiments, both biochemical and genetic, to understand the central role played by the LEAFY gene product in plant growth and pattern formation.

\section{University of California Berkeley, CA 94720}

\section{Genetic Analysis of Adh1 Regulation}

M. Freeling, Department of Plant Biology

$\$ 182,000$ (2 years)

Little is known about organ- and developmental stage-specific expression of maize Adh1. We are investigating how such expression patterns are established by identifying cis-acting sequences and their interaction with potential regulatory proteins.

Transposon-induced mutant analysis revealed the importance of the TATA region in organ-specific expression. A TATA-duplication mutant, (Adh1-3F1124), expresses ADH1 in roots and scutellum at $6 \%$ of wild type levels but at normal levels in pollen, while a TATA-deletion (18bp) mutant, (Adh1$3 F 1124 r 17)$, expresses at $60 \%$ of wild type in roots and scutellum but at greatly reduced $(30 \%)$ activity levels in pollen. This organ-specificity is reflected at different levels of gene expression (transcription vs. post-transcription) and by differential selection of aberrant transcription initiation sites. The untranslated leader region of Adh1 also appears to contain organ-specific information (at $\sim 47$ ). Mutants that carry small nucleotide changes in this region express normal ADH1 activity in roots and scutellum but pollen expression is either reduced (35\% of wild type) or increased ( $150 \%$ of wild type). The Adh1 TATA box region and the untranslated leader region (+ 47) are currently manipulated by site-specific mutagenesis to identify nucleotide requirement(s) for organ-specific expression. Expression from these mutant constructs will be tested transiently after particle bombardment into intact maize organs as well as after electroporation of tissue culture cell protoplasts. The interaction between these cis-sequences and transcription factor(s) will be initially approached by analysis of the binding properties of a maize TFIID gene recently isolated in our laboratory. 


\section{University of California Berkeley, CA 94720}

\section{Transcription Control Elements and Manipulation of Chloroplast Genes W. Gruissem, Department of Plant Biology $\quad \$ 74,250$ (9 months)}

Chloroplast gene expression in higher plants is controlled at the transcriptional and post-transcriptional level. Our work has focussed on the control elements that regulate the differential transcription activity of several chloroplast genes. We have shown that most mono- and polysistronic chloroplast transcription units contain proximal to their transcription start promoter elements that dictate the relative transcriptional activity of these genes by their respective promoter strength. In addition, the transcription of certain other genes is also regulated in response to the plant developmental program or light quality changes, but the molecular mechanisms that regulate promoter activity are not understood. To address this problem we continue our work to isolate and characterize transcription initiation complexes using affinity chromatography. The dissection of highly purified and active complexes will allow us to determine if and what factors besides the core RNA polymerase participate in the regulation of promoter activity. At the post-transcriptional level our work has concentrated on the structural and functional analysis of RNA-binding proteins that regulate the processing and/or stability of chloroplast mRNAs. We have recently isolated a $28 \mathrm{KDa}$ RNA-binding protein (28RNP) which has two conserved RNA-binding domains and an acidic $\mathrm{N}$-terminus, and which is required for 3'-terminal processing of at least four plastid mRNAs. The isolation and purification of other RNAbinding proteins is in progress. As part of this project we will analyze the structural determinants of the 28RNP that are required for RNA-binding and protein-protein interactions.

\section{University of California Berkeley, CA 94720}

\section{Determination of Genes and Phenotypes of Bacteria Necessary for Epiphytic Colonization and Survival on Plants \\ S.E. Lindow, Department of Plant Pathology

Bacteria that live as epiphytes on the surface of healthy plants are important as plant pathogens, in causing plant frost injury by catalyzing ice formation, and in other processes. The objectives of this study are to determine the traits of these epiphytic bacteria which allow them to grow and/or survive in the hostile leaf surface environment. The genes and phenotypes of a strain of Pseudomonas syringae that are necessary for epiphytic fitness on bean leaves are being determined by an evaluation of the fitness and phenotypes of 5,300 individual Tn $\underline{5}$-induced insertion mutants. Attention is being placed on several mutants which exhibit no known phenotypic alteration, but which while growing normally on moist leaf surfaces succumb to the stresses associated with dry leaf surfaces, unlike the parental strain. Two genes from stress-sensitive epiphytic fitness mutants that have individually large effects on fitness have been identified in a cosmid library of the parental $P$. syringae strain by sequence homology to Tn $\underline{5}$-containing fragments cloned from mutant strains. A $4.0 \mathrm{~kb}$ region subcloned from one cosmid restores the ability of mutant strains to survive drying stress on leaves. Fusions of these regions with a promoterless InaZ gene have been made to determine the regulation of transcription of these regions in situ by measuring the ice nuclei produced in merodiploid strains containing these fusion genes. An Ice derivative of the parental $P$. syringae strain has been made by marker-exchange mutagenesis to allow measurements of ice nuclei in situ in these merodiploids exposed to different conditions on leaves. 


\section{University of California Berkeley, CA 94720}

\section{Phytochrome from Green Plants: Assay, Purification and Characterization P.H. Quail, Department of Plant Biology}

The goal of this research program is to define the molecular properties, biogenesis, and mechanism of action of greeri-tissue phytochrome. Previous work has shown the presence of a multigene family of phytochrome (phy) genes in Arabidopsis. We have now shown by Southern blot analysis that rice contains single copies of genes encoding type $A(p h y A)$ and type $B$ (phyB) phytochromes and have isolated clones encoding the entire phyB polypeptide. This monocot sequence is more closely related to phy $B$ from the dicot, Arabidopsis (73\% amino acid sequence identity), than it is to the phyA gene in the rice genome ( $50 \%$ identity). These data support the proposal that phyA and phyB subfamilies diverged early in plant evolution and that subsequent divergence accompanied the evolution of monocots and dicots. Moreover, since rice and Arabidopsis phyB polypeptides are more closely related to one another ( $73 \%$ identity) than are monocot and dicot phyA sequences $(63-65 \%$ identity), it appears that phyB has evolved more slowly than phyA. Gene-specific Northern blot analysis indicates that whereas phyA is negatively regulated by phytochrome in rice-seedling shoots in the manner typical of monocots, phyB is constitutively expressed irrespective of light treatment, a characteristic of green-tissue or Type 2 phytochrome. In consequence, phyA and phy $B$ transcripts are equally abundant in fully green tissue. Since Arabidopsis phyB mRNA levels are also unaffected by light, our results suggest that this mode of regulation is evolutionarily conserved among phy $B$ genes, perhaps reflecting differences in the functional roles of the different phytochrome subfamilies.

\section{University of California Berkeley, CA 94720}

\section{Cloning and Characterization of Genes Determining Disease Resistance in Arabidopsis- Pseudomonas Interactions \\ B.J. Staskawicz, Department of Plant Fathology \\ $\$ 193,000$ (2 years)}

We have recently established that Arabidopsis thaliana is an excellent model plant host to study the genetic and molecular basis of plant-bacterial specificity and the expression of disease resistance (The Plant Cell 1991, 3:49-59). Our results have demonstrated that the Arabidopsis ecotype Col-0 is susceptible to the Pseudomonas syringae pv. tomato strain DC3000 and is resistant to strain JL1065. The avirulence gene avrRpt2 has been identified from a genomic library from strain JL1065 by i entifying a cosmid clone that converted the normally virulent DC3000 strain to avirulence when inoculated into Col-0. The avirulence gene is currently being characterized by subcloning and nucleotide sequencing. We are also investigating the regulation of expression by northern blot analysis.

The ecotype Po-1 has been identified that does not recognize the avirulence gene avrRpt2 and a susceptible phenotype is observed when inoculated with DC3000(avrRpt2). Genetic crosses have been constructed between $\mathrm{Col}-0$ and PO- 1 and progeny will be scored for inheritance of resistance to DC3000 carrying the avrRpt2 avirulence gene. Finally, we are screening M2 Col-0 mutagenized seed for the presence of susceptible plants that no longer recognize avrRpt2. Mutants isolated in this manner will be genetically characterized and their chromosome position identified. 


\section{University of California Berkeley, CA 94720}

\section{Analysis of Proteins Essential for Agrobacterium Mediated DNA Transfer io Plant Cells P. Zambryski, Department of Plant Biology $\$ 50,000$}

The initial goal of the proposed research was to understand how Agrobacterium transfers DNA to plant cells. The project specifically aimed to characterize the ( $v i n$ protein products that mediate this transfer event. In the last year we compleied the nucleotide sequence analysis of the virB region [Kuldau, G.A., DeVos, G., Owen, J., McCaffrey, G., and Zambryski, P. 1990. The virB operon of Agrobacterium tumefaciens pTiC58 encodes eleven open reading frames. Molec. Gen. Genet. 221, 256-266]. We are currently overproducing each of the individual virB proteins for antibody production to use in detailed biochemical and cell biological analyses to determine the location and function of the 11 virB ORFs. Since our lab has grown in size and diversity, we have initiated two new projects (acknowledging DOE support). One project aims to study how plant viral nucleic acid moves between adjacent plant cells during infection [Citovsky, V., Knorr, D., Schuster, G., and Zambryski, P. 1990. The P30 movement protein of tobacco mosaic virus is a single strand nucleic acid binding protein. Cell 60 , 637-747]. These studies demonstrated that the movement protein of tobacco mosaic virus, $\mathrm{P} 30$, is a nonsequence specific, cooperative, single strand nucleic acid binding protein. This result allowed us to formulate a model for P30 mediated viral movement wherein P30 acts as an unfoldase to create a viral nucleic acid protein complex that is extended and thin. The shape and dimensions of the complex would allow transit through narrow plasmadesmatal channels, the intercellular connections through which viral nucleic acid must pass. A second new project aims to isolate and characterize genes important during flower development in Arabidopsis.

\section{University of California Davis, CA 95616}

\section{Restriction of Virus Infections by Plants}

Plant pathogens adversely affect plants by reducing their productivity, for example of biomass. Resistance genes provide a widely used, ecologically sound, and usually economical approach to controlling pathogens. Understanding natural resistance against pathogens is expected to direct future experiments in engineering resistance against pathogens for which genetically compatible sources of resistance are not available. The Arlington line of cowpea (Vigna unguiculata) resists infection by cowpea mosaic comovirus (CPMV), a member of the comovirus group of plant viruses. In crosses with the CPMV-susceptible cowpea line Blackeye 5, resistance against CPMV was inherited as a dominant character. A seven step backcross series of Arlington to Blackeye 5 generated nearly isogenic pairs of cowpea lines, one resistant and one susceptible to CPMV. CPMV was found to protect Arlington cowpeas against certain viruses, but only if CPMV and the other virus were coinoculated. We designate this phenomenon "concurrent protection." Surprisingly, progeny of the backcross series that are resistant against CPMV also exhibited CPMV-induced concurrent protection when co-inoculated with cowpea severe mosaic comovirus, southel., bean mosaic sobemovirus, or any of three nepoviruses. The results suggest that CPMV triggers a response controlled by Ailington line-derived gene(s), and this response is able to prevent the replication anditor spread of not only CPMV but of any of several other viruses as well.Our proposed experiments are designed to identify the CPMV signals that Arlington cowpeas detect, to characterize the plant response, and to identify the Arlington line-derived genes that mediate that response. 


\section{University of California Davis, CA 95616}

\section{Modifying $\mathrm{K}^{+} / \mathrm{Na}^{+}$Discrimination in Salt-Stressed Wheat Containing Chromosomes of a Salt-Tolerant Lophopyrum \\ E. Epstein and J. Dvorak, Departments of Land, Air and Water Resources, and Agronomy and Range Science \\ $\$ 80,000$}

This project is devoted to research and development of crops more tolerant of saline soils and water than our present cultivars. This fifth year of drought in the West lends added impetus to this research, for the availability of less water amounts to an exacerbation of the salinity problem. It amounts to a diminution of the capture of solar energy by plants. Our approach is to combine genetics, specifically cytogenetics, with plant physiology to elucidate the genetic control of physiological traits associated with salinity tolerance. Potassium being the quantitatively paramount metallic element required by all higher plants, we focus on the relation between it and $\mathrm{Na}^{+}$, for $\mathrm{Na}^{+}$usually predominates in saline substrates and has the potential of swamping the $\mathrm{K}^{+}$transporting and utilizing mechanisms of plants. We experimented with wheat, Triticum aestivum (salt-sensitive), cv 'Chinese Spring', its amphiploid with tall wheatgrass, Lophopyrum elongatum (salt-tolerant), and lines of wheat containing each of the chromosomes of $L$. elongatum as substitutions for their 'Chinese Spring' homoeologous chromosomes. The plants were grown in field trials under control, intermediate, and highly saline regimes. The amphiploid is far superior in salt iolerance to 'Chinese Spring', ar J has higher $\mathrm{K}^{+} / \mathrm{Na}^{+}$ratios in its flag leaf. L. elongatum chromosome $3 E$ bestowed additional salt tolerance on wheat, as did chromosomes $2 \mathrm{E}, 4 \mathrm{E}$ and $7 \mathrm{E}$. Slight effects were elicited by chromosomes $1 \mathrm{E}$ ano $5 \mathrm{E}$. There was a general trend for added salt tolerance in wheat to be correlated with high $\mathrm{K}^{+} / \mathrm{Na}^{+}$ratios in the flag leaf.

\section{University of California Davis, CA 95616}

\section{Transposon Tagging of Disease Resistance Genes} R.W. Michelmore, Department of Vegetable Crops

We are developing a transposon mutagenesis system for lettuce to clone genes for resistance to the fungal pathogen, Bremia lactucae. Our recent efforts have been focused on the heterologous transposon Ac from corn. Transposition of wildtype Ac elements has not been detected in regenerated lettuce plants, despite analysis of numerous individuals by Southern hybridization and PCR. A variety of different constructs were used that have been shown by others to allow transposition in other species. Transposition of $A C$ has now been demonstrated in lettuce callus using an excision assay in which transposition resulted in the generation of a functional gene for resistance to kanamycin and therefore the selection of cells containing transposition events; transpos ition was stimulated by expression of the $A c$ transposase gene from the 2' promoter (Plasmids courtesy of B. Baker \& J. Ellis). Transposition was confirmed by PCR amplification and sequencing of the excision site and by Southern hybridization. Several Acpromoter::GUS fusions were made to investigate possible reasons for the infrequent transposition of $A C$ in lettuce. Comparisons between tobacco and lettuce callus showed that the level of expression was consistently lower in lettuce. The long untranslated leader seemed to reduce expression. We are currently analyzing plants containing Ac elements lacking the long untranslated leader as well as plants containing two element systems with the transposase being expressed from a heterologous promoter and the Ds, the non-autonomous element, including wholeplant selectable marker genes (Constructs courtesy of J. Jones). 


\section{University of California Irvine, CA 92717}

\section{Membrane Bioenergetics of Salt Tolerant Organisms J.K. Lanyi, Department of Physiology and Biophysics}

$\$ 168,000$ (FY 90 Funds/2 years)

Energy-generating and utilizing membrane systems are elements of a unique salt tolerant physiology in extremely halophilic archaebacteria. The proton and chloride transporting bacterial rhodopsins and the proton transporting ATPase are described. Studies of the former two systems, bacteriorhodopsin and halorhodopsin respectively, concentrate on the thermodynamics of the reaction cycles, the configuration of the immediate environment of the retinal Schiff base, and on single residues identified or assumed to play roles in the ion transfer reactions. Site-specific mutagenesis with gene expression in Halobacterium halobium and optical multichannel spectroscopy are special methods developed for this work. Studies of the latter system, the membrane ATPase of Halobacterium saccharovorum, are in the direction of cloning and the sequencing the structural gene, and of describing the catalytic ATP binding sites, the nature and causes of non-linear hydrolytic kinetics, and the shared features with eubacterial and eukaryotic ATPases.

\section{University of California La Jolla, CA 92093-0116}

\section{Structure, Biosynthesis and Role of Complex Protein-Bound Glycans M.J. Chrispeels, Department of Biology}

Plant glycoproteins contain two types of asparagine-linked oligosaccharide sidechains (glycans). Both types originate as high-mannose glycans in the endoplasmic reticulum when the proteins are first synthesized. Then, as the proteins pass through the Golgi complex, some glycans are modified by enzymes in the Golgi. These modifications result in a variety of complex glycans. We are studying the enzymes which are involved in these modifications and the sequence in which these various reactions occur. Our most recent work consists of isolating mutants of Arabidopsis thaliana that do not have complex glycans. We have screened 10,000 seedlings and found 6 whose proteins do not react with an antiserum that is specific for complex glycans. Outcrossing and selfing of these mutant plants shows that there is no observable phenotype associated with the inability of the plant to convert high mannose into complex glycans. The biochemical analysis of the glycans is now in progress. An additional project concerns the glycoprotein $\alpha$-amylase inhibitor, a protein that inhibits the $\alpha$-amylase of coleoptera. We are carrying out genetic engineering experiments to introduce the gene for this protein into potato plants.

\section{University of California Los Angeles, CA 90024-1569}

\section{Energy Capture and Use in Plants and Bacteria}

P.D. Boyer, Molecular Biology Institute

$\$ 68,058$

The principal goal of our research is to gain a better understanding of how ATP is made by the membrane-bound ATP synthase. One approach, based on measurements of the exchange of phosphate oxygens with water oxygens, provides a measure of the extent of reversal of bound ATP cleavage prior to release of $P_{1}$. This is being applied to $F_{1}$ ATPases with one catalytic site blocked with a covalent inhibitor or with one beta subunit combined with aurovertin. Results should show if one or both of the remaining two sites or subunits have activity, whether catalytic properties have been changed, and whether remaining sites show catalytic cooperativity. Other studies will explore whether 
the $\mathrm{Mg}^{2+}$ that can markedly inhibit ATPase activity may have a crucial role in the ATP synthesis reaction, whether the binding site for the $\mathrm{Mg}^{2+}$ depends on the presence of ADP at a catalytic site, and whether the binding site for the potent inhibitor azide depends on prior binding of the $\mathrm{Mg}^{2+}$. Explorations are also underway on the role of nucleotides on the noncatalytic sites, following up our recent discovery that presence of MgATP at one specific noncatalytic site can be essential for CF, ATPase activity. Preliminary experiments indicate that both activating or inhibiting effects may occur depending on whether ATP or ADP is bound and at which sites.

\section{University of California Los Angeles, CA 90024}

\section{Molecular Biology and Genetics of the Acotate-Utilizing Methanogenic Bacteria} R.P. Gunsalus, Department of Microbiology

Methanogenesis from acetate by the Methanosarcina spp. is a rate limiting step in anaerobic biodecomposition. However, the molecular and genetic basis for this process is not well understood. We are using the acetate-utilizing strain, Methanosarcina thermophila TM-1, as a model organism to examine how genes are regulated in this class of organisms in response to changes in nutrient and environmental conditions. Because this species can be cultured in a unicellular form that lacks a heteropolysaccharide layer, cells can be gently disrupted to obtain protoplasts or lysed to yield intact genomic DNA and RNA. Chromosomal DNA libraries have been constructed for $M$. thermophila and genes involved in the acetate utilization pathway including the calh genes for subunits of the carbon monoxide dehydrogenase enzyme have been cloned. They are being characterized with respect to their structural and regulatory properties. We are also examining the molecular basis for another highly regulated process, the ability of these organisms to adapt and grow over a wide range of saline conditions. We recently identified two B-amino acids, $\mathrm{N}_{\mathrm{e}}$-acetyl-B-lysine and B-glutamine, that are synthesized in response to osmotic stress, and we are examining the physiological and molecular basis for their regulated synthesis. These studies should enhance our understanding of the general regulatory mechanisms in the acetotrophic methanogens and help elucidate the adaptive processes for acetate utilization in $M$. thermophila.

\section{University of California Los Angeles, CA 90024}

31. The Gibberellin $A_{20}$ 33-hydroxylase: Isolation of the Enzyme and its Molecular Biology B.O. Phinney and J. MacMillan, Department of Biology $\$ 137,000$ (FY 90 Funds/2 years)

The primary goals of this project are: to locate the regions of highest activity for the enzyme $3 B$ hydroxylase, that catalyzes the gibberellin (GA) biosynthetic step, $\mathrm{GA}_{20}$ to $\mathrm{GA}_{1}$; to purify the enzyme to a level where its biochemical properties can be studied; to compare the properties of the wild type enzyme in maize to those of the $d w a r f-1(d 1)$ mutant of maize. (The $d 1$ mutant controls the step $\mathrm{GA}_{20}$ to $\mathrm{GA}_{1}$ presumably through the production of an altered enzyme); to study gene expression using normal and $d 1$ cloned genes.

We have successfully located a region of the stem (the nodes) that has 50 to $100 \mathrm{X}$ the activity of the stem internode (the region that was used in our original preliminary studies). Our assay involves the release of tritium from $1 B, 2 B, 3 B$-tritium labelled $G_{20}$; in the presence of the enzyme the $3 B$ position of $\mathrm{GA}_{20}$ is hydroxylated with the release of the tritium label which becomes incorporated into the water of the medium. The assay requires $24 \mathrm{~h}$; up to 200 assays can be run at any one time. Typical observed values are: background, $50 \mathrm{cpm}$; internodes $290 \mathrm{cpm} / \mathrm{gfw}$; nodes $13,250 \mathrm{cpm} / \mathrm{gfw}$. Low values are found throughout the internode with a sharp increase in activity at the node; values drop 
abruptly just above and below the nodes. High activities are observed from both old and very young nodes. High activity has not been observed from leaf tissues. We use $0.1 \mathrm{~g}$ to $1.0 \mathrm{~g}$ of tissue slices ( $1 \mathrm{~mm}$ thick) incubated in $2.0 \mathrm{~mL}$ of $0.1 \mathrm{M}$ Tris, $\mathrm{pH} 7.6,10 \mathrm{mM}$ ascorbate, $2.5 \mathrm{mM}$ a-ketoglutarate and $1.0 \mathrm{mM} \mathrm{FeSO}_{4} ; 500,000 \mathrm{dpm} \mathrm{GA}_{20}$ is used in each assay.

\section{University of California \\ Los Angeles CA 90024-1606}

\section{2. $\mathrm{CO}_{2}$ and the Stomatal Control of Water Balance and Photosynthesis in Higher Plants E. Zeiger, Department of Biology $\$ 150,000$ (FY 90 Funds/2 years)}

This research program studies the stomatal responses to $\mathrm{CO}_{2}$ and their interactions with other environmental signals modulating stomatal movements. Characterization of the mechanisms regulating the $\mathrm{CO}_{2}$ responses is important for the understanding of gas exchange in leaves and for evaluations of the impact of global climate changes on the vegetation. Guard cell chloroplasts

operate the phorosynthetic carbon fixation pathway and we are currently investigating whether carbon fixation in guard cells can mediate stomatal responses to $\mathrm{CO}_{2}$. Sugars and organic acids are quantified by HPLC in guard cells from epidermal peels, guard cell protoplasts and isolated guard cell chloroplasts, and light quality is used to stimulate alternative pathways of carbon metabolism. Under low fluence rates of blue light, guard cells accumulate malate, citrate, sucrose and other products of starch breakdown such as maltose. Under red light, guard cells show very little organic acid or maltose accumulation, and accumulate mainly sucrose. Isolated guard cell chloroplasts arcumulate malate when stimulated by blue light. These biochemical probes will be used to characterize guard cell osmoregulation and stomatal opening under solar radiation, and its modulation by $\mathrm{CO}_{2}$.

\section{University of California Santa Cruz, CA 95064}

\section{Tonoplast Transport and Salt Tolerance in Plants L. Taiz, Biology Department}

Tonoplast transport driven by the tonoplast $\mathrm{H}^{+}$-ATPase plays a vital role in salt tolerance in many plant species. Our studies have been aimed at understanding how the tonoplast ATPase is regulated in plant cells. Previously we characterized the promoter region of the gene for the catalytic A subunit of the carrot tonoplast ATPase and demonstrated the presence of three different transcripts produced by the same gene (Struve et al., 1990). Recently we obtained evidence for three different genes representing isoforms of this subunit. The three genes were identified from PCR products of genomic DNA. The isoform genes differ mainly in their introns. Western blots of 2-D gels of carrot microsomal membranes probed with antibody to the A subunit revealed three spots. Moreover, carrot cells transformed with antisense cDNA to the A subunit of the tonoplast ATPase were missing two of the spots. The activity of the tonoplast ATPase was inhibited in the antisense transformants, but the Golgi ATPase was unaffected. We are currently investigating the possibility of tonoplast-specific and Golgispecific isoforms. 


\section{University of Chicago Chicago, IL 60637}

\section{Organization and Regulation of the Genes for Nitrogen Fixation in Rhodobacter capsulatus \\ R. Haselkorn, Department of Molecular Genetics and Cell Biology \\ $\$ 89,000$}

We are continuing to study the control circuits that regulate expression of the genes for nitrogen fixation in the photosynthetic bacterium Rhodobacter capsulatus. The two environmental factors known to control expression of the genes encoding the structural components of nitrogenase are oxygen and a source of combined nitrogen, such as ammonia. Nitrogen source control of nif gene expression is mediated by the products of the nifR1, nifR2, nifR4, nifR5, and nifA genes. Mutations in nifR5, which encodes protein PII, a regulator of adenylyl transferase, are constitutive with respect to ammonia. Mutations in the other four genes create a Nif phenotype. NifR1 and NifR2 are required to activate transcription of nifR4, which encodes a sigma factor. NifR4, as well as NifR1 and NifR2, are required to transcribe nifA; NifA and NifR4 are needed to transcribe nif structural genes. Oxygen inactivates the NifA protein. We believe that oxygen has additional effects on nif gene expression mediated by the state of DNA supercoiling. To explore the latter possibility, we have used 177-bp PCR products provided by W. Huang, complementary to the gyrB gene of $R$. capsulatus, to clone two copies of the gyrB gene. These are currently being sequenced and prepared for site-specific mutagenesis. One of the cosmids containing a gyrB gene turns out to contain, in addition, a gene whose transcription requires NifR4. The nature of this gene is being determined.

The preparation of a low resolution physical map of the $R$. capsulatus chromosome, thought to have been completed by others, has been undertaken anew. The sum of fragment sizes for several enzymes is approximately $4 \mathrm{Mb}$. A good unambiguous map covering half the chrorinosome has been completed; no problems are anticipated for the remainder. A device for accurate large scale replica plating and blotting has been constructed, in order to simplify extension of the physical map resolution to the level of fragment sites in cosmids. We would be grateful for gifts of cloned $R$. capsulatus genes in order to locate them on the physical map.

\section{University of Chicago Chicago, IL 60637}

\section{Signal Transduction in Plant Development: Chemical and Biochemical Approaches to Receptor Identification \\ D.G. Lynn, Department of Chemistry \\ $\$ 176,000$ (2 years)}

Striga asiatica is a small chlorophyll-containing angiosperm that has developed the remarkable ability to establish a vascular connection with another plant. The development of the attachment organ, the haustorium, is one of the most rapid organogenesis events known. Since this parasitic ability is widespread among the plant families, it has generally been assumed that the development of the attachment organ relies on ubiquitous plant developmental programs. It was therefore quite surprising when it was discovered that relatively simple host-derived phenolic compounds were sufficient to induce haustorial development. The subsequent discovery that simple phenolic compounds had evolved as the primary signal initiating vir expression in Agrobacterium and nod expression in Rhizobium and that some of these same compounds were important in plant growth and development have raised fundamentally new questions about the role of these compounds in plants. 
Subsequent work on the phenolic signals in Striga has provided evidence that the compounds are detected via a chemical reaction, again quite distinct from our current models of hormone/growth factor detection by membrane localized binding proteins. Evidence is presented that the recognition mechanism is a redox reaction most likely controlled by plasma membrane localized oxidoreductases. While the existence of these redox systems have been demonstrated in hoth plants and animals, only recently has convincing evidence connecting $e$ - transport with plant development emerged.

Striga therefore can teach us not only about the development of host-parasite interactions but also represents a rapid and well defined developmental transition where the chemistry of the inducing signal can be simply exploited to provide in sights into the developmental process. We propose to use this chemistry both to provide information about the initial detectors controlling haustorial development and to help in the exploitation of the molecular biology of Striga.

\section{Clemson University Clemson, SC 29634-1903}

36. The Magnesium Chelation Step in Chlorophyli Biosynthesis J.D. Weinstein, Department of Biological Sciences

In photosynthetic organisms, the biogenesis of energy transducing membranes requires the coordinate synthesis of prosthetic groups, proteins, and various lipids. Two of the major prosthetic groups, chlorophyll and heme, share a common biosynthetic pathway that diverges at the point of metal insertion into protoporphyrin IX (Proto). Insertion of iron leads to the formation of hemes, while insertion of magnesium is the first step unique to chlorophyll formation. This project is directed toward identifying the enzyme(s) responsible for magnesium chelation and elucidating the mechanism which regulates the flux of precursors through the branch point enzymes in isolated chloroplasts. Using intact chloroplasts from greening cucumber cotyledons, we have confirmed the ATP requirement for $\mathrm{Mg}$-Proto formation. Use of non-hydrolyzable ATP analogs, uncouplers and ionophores has led to the conclusions that ATP hydrolysis is necessary, but that this hydrolysis is not linked to the requirement for membrane intactness by transmembrane ion gradients or electrical potentials. The enzyme(s) are flexible with respect to the porphyrin substrate specificity, accepting porphyrins with -vinyl, -ethyl or $-H$ substituents at the 2 and 4 positions. The activity increases approximately four-fold during greening. Possible physiological feedback inhibitors such as heme, protochlorophyllide, and chlorophyllide had no specific effect on the activity. The activity has now been assayed in barley, corn and peas, with the system from peas almost ten-fold more active than the cucumber system. Work is continuing in pea chloroplasts with the development of a continuous assay and investigation of the feasibility of characterizing an active, organelle-free preparation.

\section{Cold Spring Harbor Laboratory Cold Spring Harbor, NY 11724}

\section{Suppression of Mutations Generated by Mu Transposons in Maize R. Martienssen \\ $\$ 144,000$ (2 years)}

Transposable elements of the Robertson's Mutator family are highly active in the maize genome, and are widely used for "transposon tagging". However, in some cases phenotypic expression of Mu induced mutants depends on the activity of the Mutator system. In the case of the non-photosynthetic seedling lethal mutant hcf106, a Mu1 transposon has inserted near the site of transcript initiation. When $M u$ is in an active phase, transcripts from the mutant allele fail to accumulate. When plants lose Mu activity, transcripts initiate from a novel promoter formed from the junction between the Mu1 element and the gene. This results in suppression of the mutant phenotype as the resulting message 
can encode a functional gene product. DNA methylation of Mu elements is also correlated with phenotypic suppression, and may be involved in establishing or maintaining altered transcriptional states. There are three other examples of $M u$-suppressible mutations at cloned loci in maize, and in each one there is a Mu1 insertion close to the start of transcription, suggesting a common mechanism in each case.

We will characterize the mechanism of phenotypic suppression by $M u$, using hcf106 as a model system. We will investigate the role of cis- and trans-acting factors that influence the activity of the novel promoter found in $M u$ ends: at least three different DNA binding factors interact with the $M u$ ends, and some of these are likely to influence suppression. Plants carrying the hcf106 mutation can lose Mu activity somatically, resulting in sectors of phenotypically suppressed tissue. These sectors progressively increase in size in successive leaves. We will determine the genetic and developmental basis for this pattern of expression by in situ hybridization, and by making double mutants with a variety of mutations in maize that affect the pattern of normal development. This research will help us to understand interactions between transposable elements and their host genome, and may lead to more efficient schemes for transposon-tagging and gene isolation.

\section{Cornell University Ithaca, NY 14853}

\section{Anaerobic Metabolism of Aromatic Compounds by Phototrophic Bacteria: Biochemical Aspects J. Gibson, Division of Biological Sciences

Very large quantities of aromatic compounds, many of them recalcitrant and potentially carcinogenic, are released into the biosphere as industrial byproducts. Lignins derived from plant sources are an even larger source of benzenoid rings in nature. Many of these compounds find their way into anaerobic environments, where they can be degraded, if slowly, by anaerobic bacteria. Little is known concerning the metabolic pathways, let alone the detailed enzymology or regulation, employed in these processes. The major objectives of this project are to elucidate the biochemistry of the degradation of benzoate and of 4-hydroxybenzoate by the phototropic bacterium Rhodopseudomonas palustris, one of the relatively small number of microorganisms known to grow well with these and related compounds as sole carbon source. We have shown that these two compounds, or close derivatives, appear to be key intermediates in the degradation of a range of more complex aromatic acids utilized for growth. The first step in the anaerobic attack on these model compounds involves the formation of Coenzyme A thioesters. Two immunologically unrelated CoA ligases have been purified so far, yielding antisera and protein sequence infe"mation which should allow us to clone these key enzymes. They will also be used to prepare radioactive substrates for the subsequent reductive reactions of the pathway. Major efforts will be made in the coming year to define the number of enzymes and cofactors involved in reductive saturation of the aromatic ring, using a number of mutants with defects in aromatic substrate utilization as well as wild type cells and cell extracts. The overall biochemistry is likely to be similar in other types of microorganisms that degrade aromatics anaerobically, so that these studies will contribute ultimately to possibilities for engineering more rapid or diversified biodegradations in such environments. 


\title{
Cornell University Ithaca, NY 14853
}

\author{
39. Molecular Analysis of Cytoplasmic Male Sterility \\ M.R. Hanson, Section of Genetics and Development
}

The ultimate aims of the project are to understand the molecular mechanism of the disruption in pollen development which occurs in cytoplasmic male sterile plants and to understand the control of respiratory energy flow in the higher plant cell. A mitochondrial locus termed $S$-pcf segregates with sterility and with an alteration in respiration in Petunia. This cloned locus contains three genes, an abnormal fused gene termed pcf, a gene for a subunit of an NADH dehydrogenase complex, and a small ribosomal subunit protein. The pcf gene is comprised of partial sequences of ATPase subunit 9. cytochrome oxidase subunit II, and an unidentified reading frame. Components of the S-Pcf locus will be introduced into the nucleus of a fertile genotype under the control of appropriate regulatory signals, and polypeptide products of introduced genes will be directed to the mitochondrion with a transit peptide. By examining transgenic plants, we can determine what elements of the locus are critical for altered respiration or sterility. Such knowledge could explain how mitochondrial DNA affects poller development in the large number of plant species which exhibit the agronomically important trait of miale sterility.

\section{Cornell University Ithaca, NY 14853-1902}

\section{Mechanisms and Genetic Control of Interspecific Crossing Barriers in Lycopersicon M.A. Mutschler, Department of Plant Breeding \& Biometry \\ S. McCormick, USDA Gene Expression Lab, Albany, CA \\ $\$ 142,000$ (2 years)}

Several mechanisms limit cross fertilization among species. Interspecific crossing barriers can result in poor pollen tube growth, lack of fertilization, reduced embryo survival or seed viability in the interspecific cross or its $F_{1}$ or $F_{2}$, and in aberrant segregation ratios in interspecific $F_{2}$ 's. The interspecific barriers affect the exchange of genes between species, and impedes the transfer of desirable multigenic traits from unadapted and wild germplasm to crop species. Since many of the traits needed for crop improvement are multigenic in nature, it is imperative to understand the nature and genetic control of the interspecific barriers. We have assembled a unique combination of plant materials, including interspecific layer chimeras, isocytoplasmic lines and transformants with a pollenspecific reporter gene. We will study the interspecific barriers using these materials and a combination of cytological, genetic and molecular techniques. Our goals are to determine: 1) the functional basis of unilateral incongruity including the time and developmental step(s) interrupted and the tissue and genomes involved, 2) the functional basis of hybrid breakdown (HB), the chromosomal regions associated with $\mathrm{HB}$, and whether the effects of cytoplasm on nonfecundity are direct or indirect, and 3) the functional basis of interspecific aberrant ratio syndrome (IARS) including the stage of reproductive development affected, the tissues and genomes involved, and the basis of cytoplasmic effects on IARS. The long term goals of this project are to study mechanisms controlling interspecific reproductive barriers and to use the information and materials produced to facilitate the transfer of desired quantitative traits. 


\title{
Cornell University Ithaca, NY 14853-5908
}

\author{
41. Structure and Function of the Self-Incompatibility Proteins of Brassica oleracea \\ J.B. Nasrallah, Division of Biological Sciences \\ $\$ 176,000$ (2 years)
}

The specificity of the pollen-stigma interaction in self-incompatible species belonging to the mustard family, the Brassicaceae, is controlled by more than fifty alleles at the Slocus. In Brassica, where the pollen reaction is sporophytically determined, pollen fails to produce a normal pollen-tube on stigmas expressing the same $S$ allele as the pollen parent. Our research has focused on the analysis of the products of the $S$-locus in stigma and pollen. We have characterized the highly polymorphic $S$-locus specific glycoproteins which are encoded by allelic sequences that map to the SLG (S-locus glycoprotein) site within the $S$ locus. The $S L G$ gene is expressed at high levels in the stigmatic papillae and to a lesser extent in the anther. In the stigma, the $S$-locus specific glycoproteins have been localized by immunocytochemistry and shown to accumulate in the papillar cell wall. In anthers, the expression of SLG in the tapetum and in the microspores has been revealed at the level of RNA blot analysis and in Brassica plants transformed with a chimeric gene consisting of the promoter of the SLG gene fused to the reporter gene B-glucuronidase. Future research will involve the identification of the pollen SLG proteins, and the characterization of a putative receptor protein kinase which we have recently identified and which is encoded by a second S-locus linked gene. We will investigate the activity of this putative receptor protein kinase, attempt to identify its ligands and substrates, and determine its role in the pollen-stigma interaction of self-incompatibility.

\section{Cornell University Ithaca, NY $\$ 4853$}

\section{Mechanisms of Inhibition of Viral Replication in Plants P. Palukaitis, Department of Plant Pathology}

Viruses are a major class of plant pathogens that are responsible for crop losses and reductions in plant biomass. In some cases, natural resistance genes are known that can obviate the effects of such pathogens, although none of these resistance genes have been isolated. In other cases, viral sequences have been integrated into plant genomes to protect these plants against infection. In neither case is the mechanism of action understood. This pioject is concerned with analyzing the molecular mechanisms of inhibition of viral replication and movement in plants, and how viruses mutate to overcome such resistance mechanisms. Several approaches are being used to examine the above mechanisms: (1) Transgenic plants expressing either tobacco mosaic virus or cucumber mosaic virus (CMV) movement genes are being used to determine the nature of the interaction between viral movement gene products and host genes for resistance to viral infection. (2) Transgenic plants expressing the CMV coat protein gene are being used to determine both the limitations and the mechanism of inhibition of virus infection against CMV strains. (3) Biologically-active cDNA clones of resistance-breaking and replication-defective strains of CMV are being used in concert with nucleotide sequence analysis and site-directed mutagenesis, to determine the location and nature of sequence alterations specifying host range, rate of replication, and pathogenicity. Our results indicate that as little as one or two amino acid alterations in various genes of CMV can have a significant effect on the above properties. 


\section{Cornell University Ithaca, NY 14853}

\section{Effects of Freezing and Cold Acclimation on the Plasma Membrane of Isolated Cereal Protoplasts}

P.L. Steponkus, Department of Soil, Crop and Atmospheric Sciences

$\$ 90,700$

The goal of our program is to provide a mechanistic understanding of the cellular and molecular aspects of freezing injury and cold acclimation from a perspective of the structural and functional integrity of the plasma membrane. The current focus is on dehydration-induced alterations in the plasma membrane of protoplasts isolated from cold-acclimated ye leaves in order to determine the factors that limit the maximum freezing tolerance of rye. Studies are in progress to test the hypothesis that cold acclimation increases the hydration of plasma membrane lipids such that lamellar-tohexagonal phase transitions are precluded during freeze-induced dehydration because the critical level of dehydration occurs at temperatures below the lamellar-to-hexagonal "phase transition temperature. It is predicted that severe freeze-induced dehydration of cold-acclimated protoplasts at the $L T_{50}\left(-20\right.$ to $\left.-30^{\circ} \mathrm{C}\right)$ will result in liquid crystalline-to-gel $\left(L_{\alpha}-t_{0}-L_{B}\right)$ rather than lamellar-to-hexagonal phase transitions. Under these conditions, injury is associated with a high incidence of deviations in the fracture plane from the mid-plane of the plasma membrane to aparticulate lamellae that are in close apposition to the plasma membrane; the size of the region involved ranges from 0.1 to $2.5 \mu \mathrm{m}$ in diameter. We propose that the fracture-jump phenomenon is a consequence of the formation of localized domains of asymmetric lipid species that become interdigitated after the $L_{\alpha}$-to- $L_{\beta}$ phase transition and that glucocerebrosides play a key role in this phenomenon.

\section{Cornell University Ithaca, NY 14853-8101}

\section{Genetic Control of Nitrate Assimilation in Klebsiella pneumoniae V.J. Stewart, Section of Microbiology}

$\$ 153,012$ (2 years)

Klebsiella pneumoniae is an enteric bacterium closely related to Escherichia coli. Nitrate and nitrite are important alternate nitrogen sources not only for $K$. pneumoniae, but also for many other microorganisms and for most plants. In the absence of ammonium, nitrate (or nitrite) induces the synthesis of assimilatory nitrate reductase and assimilatory nitrite reductase, which act in sequence to convert nitrate to ammonium. Our work will focus on understanding the physiological and molecular mechanisms by which ammonium and nitrate regulate the synthesis of nitrate assimilation enzymes. We have identified and cloned two genes, nas $A$ and nas $B$, that encode assimilatory nitrate and nitrite reductases, respectively. Transcription of nas-lacZ operon fusions is induced by nitrate or nitrite in the absence of ammonium. This nitrate induction does not require narL, a regulatory gene that mediates nitrate induction or respiratory nitrate reductase synthesis. We plan to examine the molecular genetics of the nas $A B$ operon by identifying its gene products and by determining the DNA sequence of the nas region. The promoter region of the nas operon will be identified and analyzed by deletion and mutational analysis, and by transcript mapping. The genetic basis for both general nitrogen regulation as well as for nitrate and nitrite induction of nas gene expression will be explored. Finally, the relationship of nas genes to the genes for respiratory nitrate reductase (nar) will be evaluated. Together, these studies will lead to a more detailed view of how nitrogen metabolism is coordinated and regulated. 


\section{Cornell University Ithaca, NY 14853}

\section{Studies of the Genetic Regulation of the Thermomonospora Cellulase Complex D.B. Wilson, Department of Biochemistry, Molecular and Cell Biology}

$\$ 142,000$ (FY 90 Funds/2 years)

The goals of this project are to determine the molecular mechanisms regulating cellulase synthesis in the soil bacterium Thermomonospora fusca and to determine the molecular mechanism by which $T$. fusca cellulases degrade crystalline cellulose. We have succeeded in obtaining crystals of the $E_{2}$ catalytic subunit that diffract to $1.8 \mathrm{~A}^{\circ}$ and have obtained an isomorphous heavy atom derivative of this crystal. The four cysteine residues in $E_{2}$ have been shown to be present in two disulfide linkages: one joining Cys 81 to Cys 126 and the other Cys 233 to Cys 308 . The fully reduced enzyme still has CMCase activity but less than the native enzyme. $E_{2}$ is a dimer even though all the other four $T$. fusca cellulases we have studied are monomeric. An $E_{2}$ mutant has been isolated that has higher activity on CMC than native $E_{2}$ but which does not dimerize; in this mutant Glu 294 is changed to Gly. Even though this Glu is conserved in all members of cellulase family $B$, it is not required for activity on CMC. Studies comparing the cellulose binding activity of $E_{2}$ and the $E_{2}$ catalytic subunit show that both forms bind to cellulose, but the affinity of $E_{2}$ is much higher than that of the catalytic subunit. The activities of these two enzymes are identical on CMC but the catalytic subunit has about $60 \%$ of the activity of the native enzyme on filter paper. ' $n$ order to obtain crystals of the $E_{5}$ catalytic subunit, we have constructed a recombinant plasmiu that is missing a sfgment of the $E_{5}$ gene that encodes the cellulose binding domain. This plasmid has been introduced into a protease negative mutant of Streptomyces lividans which we have isolated. We are currently testing this system to see if we can obtain large amounts of a homogenous preparation of the $E_{5}$ catalytic subunit for crystallization.

\section{Cornell University Ithaca, NY 14853-7201}

\section{Conversion of Acetic Acid to Methane by Thermophiles}

The objective of this project is to provide an understanding of thermophilic anaerobic microorganisms capable of breaking down acetic acid, the precursor of two-thirds of the methane produced by anaerobic bioreactors. Recent results include: 1) the isolation of Methanothrix strain CALS-1, which grows much more rapidly than mesophilic strains; 2 ) the demonstration that thermophilic cultures of Methanosarcina and Methanothrix show minimum thresholds for acetate utilization of 1-2.5 $\mathrm{mM}$ and 10-20 $\mu \mathrm{M}$ respectively, in agreement with ecological data indicating that Methanothrix is favored by low acetate concentration; 3 ) the demonstration of high levels of thermostable acetyl-coA synthetase and carbon monoxide dehydrogenase in cell-free extracts of Methanothrix strain CALS-1; 4) the demonstration of methanogenesis from acetate and ATP in cell free extracts of strain CALS-1. Methanogenesis occurred at a high rate $(>50 \mathrm{mU} / \mathrm{mg}$ protein), required $2 \mathrm{ATP} / \mathrm{methane}$, and, in contrast to Methanosarcina, was independent of hydrogen and other electron donors; 5) the isolation in axenic culture of the acetate-oxidizing rod-shaped member (AOR) of a thermophilic two-membered coculture which converts acetate to methane via interspecies hydrogen transfer. The AOR was found to be an acetogen; 6) the finding that entropy effects must be considered when predicting the level of hydrogen in thermophilic syntrophic cultures. This prediction has been verified by others and has a considerable impact on the modeling of electron transfer in thermophilic anaerobic bioreactors; 7$)$ the isolation and characterization of the Desulfotomaculum thermoacetoxidans. Current research is centered on factors which allow thermophilic Methanothrix to compete with Methanosarcina. 


\section{Dartmouth College Hanover, NH 03755}

\section{Regulation of Gene Expression in the Bradyrhizobium japonicum/soybean symbiosis M.L. Guerinot, Department of Biological Sciences \\ $\$ 87,000$}

The importance of iron as a virulence factor in animal/pathogen interactions is well-established; the role of this metal in plant/bacterial associations is just beginning to be explored. Our studies are directed at understanding the role of iron in regulating the symbiosis between Bradyrhizobium japonicum and soybeans. Iron may be an important regulatory signal in planta as the bacteria must acquire iron from their plant hosts and iron-containing proteins figure prominently in all the nitrogen-fixing symbioses. For example, the bacterial partner is believed to synthesize the heme moiety of leghemoglobin which may represent as much as $25-30 \%$ of the total soluble protein in an infected plant cell. For this reason, we have focused our attention on the regulation by iron of the first step in the bacterial heme biosynthetic pathway. The enzyme which catalyzes this step, $\Delta$-aminolevulinic acid synthase, is encoded by the hemA gene, which we had previously cloned and sequenced. We have now documented a three to ten-fold induction in the activity of a $B$. japonicum hemA-lacZ fusion in response to iron. We have also shown that this fusion appears to be regulated by fur or by a gene controlled by fur in $E$. coli. Fur is a well characterized iron regulatory protein. We propose to define the 5' and $3^{\prime}$ cis-acting sequences which confer iron regulation on the hemA gene. We will also identify genes which encode trans-acting factors which regulate hemA expression using a variety of screening and selection strategies. Finally, we hope to identify other genes which are transcriptionally responsive to changes in iron availability. These studies will shed light on how heme synthesis is regulated in nodules and will contribute to an overall understanding of the regulation of gene expression by metals.

\section{University of Delaware Lewes, DE 19958-1298}

\section{Metabolic Mechanisms of Plant Growth at Low Water Potentials J.S. Boyer, College of Marine Studies}

In higher plants, growth is more frequently limited by water than by any other environmental resource. The project objective is to identify the underlying molecular mechanisms causing growth limitations by focusing on the process of cell enlargement. Studies so far have shown that, in localized growing regions in germinating soybean seedlings, turgor in most of the cells was completely maintained when water potentials were low enough to inhibit growth. However, gradients in water potential decreased between the vascular tissues and the enlarging cells and this was the first event to inhibit growth. The extensibility of the cell walls decreased enough to be inhibitory a few hours later and a $28 \mathrm{kD}$ protein accumulated in the walls of these cells. The protein did not accumulate in the mature tissue of soybean stems, nor in the roots where growth continued unabated. Antibodies to this protein showed an immunologically related protein in the cytoplasm. Using the cDNA for the $28 \mathrm{kD}$ protein as a probe, we observed that the mRNA for the 28kD protein increased in cytoplasm when water potentials inhibited growth, but no increase occurred in mature stem tissue or roots. In contrast, the mRNA for a similar $31 \mathrm{kD}$ protein accumulated in the roots but not in the shoots. The correlation between the wall protein, the mRNAs and the growth inhibition at low water potential suggests that the protein could play a role in the growth response. 


\section{Duke University \\ Durham, NC 27706}

\section{Stable Isotope Fractionation in Photosynthesis \\ C.B. Osmond, Department of Botany}

$\$ 90,000$

Having established analytical systems for isotope fractionation, emphasis has been given to the production of stable, homoplasmic transformants of Chlamydomonas reinhardtii for the psbA gene (previous transformations were heteroplasmic). A successful co-transformation procedure was devised using as donor the DNA fragment Xho 8 . This encompasses $11 \mathrm{~Kb}$ of the chloroplast genome and was cloned from strain CC-64 carrying the chloroplast erythromycin mutation er-u-la (er) into a plasmid. The fragment includes the entire wild type psbA gene, the 5S rRNA gene and the small exon of thie 23S rRNA gene in which the er mutation is located. Cells were transformed using the biolistic method (Boynton et al., 1988) and three different selection conditions were applied; for either photosynthetic competence or erythromycin resistance, or for both. DNA from 12 transformants was examined and in all transformants analyzed the donor DNA integrated in a pattern identical or very similar to the pattern observed for homologous sequences in the chloroplast genome of the donor strain. Sub-fragments of the above clone will be cloned in $M 13$, mutagenized and re-inserted in the original clone for transformation. Selection of transformants will be done using the er marker, after which they will be screened for psbA transformation events.

When grown with $5 \% \mathrm{CO}_{2}$ as sole carbon source, $\delta^{13} \mathrm{C}$ values of wild type, recipient and the stable psbA transformant T37-32-A1 were the same $\left(-22 \%\right.$; source $\mathrm{CO}_{2}+2 \%$. This suggests little diffusional limitation to $\mathrm{CO}_{2}$ fixation by Rukisco in these cultures. When cells were grown in $5 \% \mathrm{CO}_{2}$ plus acetate, the transformant was closer to the $\delta^{13} \mathrm{C}$ value of the heterotrophic control than either wildtype, consistent with indifferent autotrophy under these conditions. These observations confirm earlier plate experiments with the heteroplasmic transformant VIII 14-2 and pave the way for analyses of photosynthetic competence using $\delta^{13} \mathrm{C}$ and $\delta \mathrm{D}$ values as criteria for autotrophy. In this respect significant technical progress has been made with the successful on line measurement of $\delta^{18} \mathrm{O}$ value in $\mathrm{H}_{2} \mathrm{O}$ during photosynthetic $\mathrm{O}_{2}$ evolution in vivo.

\section{Duke University \\ Durham, NC 27706}

\section{Molecular Studies of Functional Aspects of Higher Plant Mitochondria J.N. Siedow, Department of Botany $\$ 148,000$ (FY 90 Funds/2 years)}

Mitochondria isolated from $\mathrm{cms}$-T lines of maize are sensitive to a toxin (BmT-toxin) derived from the fungus Bipolaris maydis, race T. Toxin sensitivity is associated with a mitochondrial-encoded $13 \mathrm{kDa}$ protein, URF13, that interacts with BmT-toxin to produce pores within the inner mitochondrial membrane. The goal of this research (carried out in collaboration with C.S. Levings, North Carolina State University) is to characterize the mechanism by which URF13 and BmT-toxin interact to permeabilize biological membranes. The expression of URF13 in E. coliconfers BmT-toxin sensitivity on the resulting bacterial cells, and binding studies with radiolabeled BmT-toxin have established that toxin binds specifically to $E$. coli expressing URF13. Crosslinking studies have established the oligomeric nature of URF13 within the membrane and computer modeling has been used to develop a model whereby each URF13 molecule is localized in the membrane through a set of three 
membrane-spanning helices. URF 13 molecules containing epitopes at both the $\mathrm{N}$ - and $\mathrm{C}$-termini have been constructed in $E$. coli. These constructs are being used to determine the topological orientation of URF13 in the membranes of both $E$. coli and $c m s-T$ mitochondria as well as confirm the validity of the three-helix model. Finally, attempts are being made to purify URF13 with the goal of reconstituting URF13 iito liposomes to develop a more well-defined system for looking at URF-13/BmT-toxin interactions.

\section{Florida State University}

Tallahassee, FL 32306

\section{Guard Cell Biochemistry - Response to Environmental Stimuli Causing Changes in Gas Exchange \\ W.H. Outlaw, Jr., Department of Biological Science \\ $\$ 70,000$}

Stomatal aperture size is regulated to effect a compromise between the opposing priorities of avoiding water loss and of admitting $\mathrm{CO}_{2}$. Pore enlargement is brought about by swelling of the subtending guard cell pair, a result of accumulation of solutes from the apoplast and synthesis of low MW substances. The specialized metabolism that these cells have evolved to fulfill their crucial role in the plant's physiology extends to more subtle aspects. Our current work has been in three areas: (a) Following up on DOE-supported findings that different leaf cells differentially accumulate ABA after stress imposition, we have attempted to determine which leaf cells synthesize ABA. Our experimental approach was to use young leaves, which have been reported to lack the ability to synthesize $A B A$. (The rationale was that a cell's accumulation of ABA would most likely have resulted from $A B A$ distribution in these leaves.) Under our conditions, we have found that young leaves of Vicia-even ones so young that stomata are not differentiated--do not predictably lack the ability to synthesize ABA. (b) We have completed our studies of cytosolic malate concentration. A direct measurement of this number is of paramount importance for several reasons (e.g., inhibition of phosphoenel pyruvate carboxylase, $\mathrm{pH}$ buffer). (c) The potential for Fru 6-P phosphorylation in guard cells has been compared with that of palisade cells. We speculate that one glycolytic sequence may be responsive to energy demand and the other to a carbon-skeleton demand.

\section{University of Florida \\ Gainesville, FL 32611}

\section{Ethanologenic Enzymes of Zymomonas mobilis}

Zymomonas mobilis is an obligately fermentative, Gram-negative bacterium capable of rapid and efficient conversion of glucose into ethanol. Although this organism is essentially prototrophic, the thirteen glycolytic and ethanologenic enzymes constitute $30 \%$ to $50 \%$ of total soluble proteins and provide a glycolytic flux in excess of 1 micromole/minute per $\mathrm{mg}$ of cell dry weight. High levels of these central pathway enzymes are essential for ATP production. The differential expression of these central pathway enzymes and the many enzymes involved in biosynthesis represents one of the most fundamental forms of regulation, balancing the enzymatic requirements for energy generation against the need to divert a portion of the glycolytic intermediates to serve as carbon skeletons for biosynthesis.

Over $95 \%$ of the glucose metabolized by this organism is converted to ethanol and $\mathrm{CO}_{2}$, both readily measured products of fermentation. This coupled with the lack of allosteric control in the glycolytic enzymes make $Z$. mobilis an attractive organism for investigations of glycolytic flux and flux controls. 
All of the major glycolytic enzymes in $Z$. mobilis have now been cloned and most have been sequenced. These are organized into two polycistronic and seven monocistronic operons. Each is present as a single chromosomal copy. Although all data are not complete, a comparison of features indicates that most have a highly biased pattern of codon usage, a canonical ribosomal-binding site, tandem promoters, an untranslated leader sequence, and missages which are quite stable. All of gene products have been resolved on 2-dimensional PAGE gels and ranked as to relative levels of expression. Message stability appears to be the primary feature which determines relative expression among these abundant gene products. Message stability is hypothesized to be the principal determinant which ensures high level expression of the thirteen genes in central metabolism as compared to biosynthetic genes.

The differential expression of gap and pgk in the gap operon is being investigated in some detail. Higher levels of glyceraldehyde-3-phosphate dehydrogenase are required for glycolysis due to the lower catalytic efficiency of this enzyme. The three to four-fold higher levels of the gap gene product result from increased translation of the more stable gap segment of the message. Stem-loop structures at both the $3^{\prime}$ and $5^{\prime}$ ends of the gap message appear to be involved in this stabilization.

Although $Z$. mobilis is an extremely efficient ethanologenic microorganism, the substrate range of this organism is limited to glucose and fructose. Genes cloned from $Z$. mobilis and fundamental information regarding high level gene expression are being used to develop recombinant strains of other organisms for fuel ethanol production.

\section{University of Florida}

Gainesville, FL 32611

\section{Gene-enzyme Relationships of Aromatic Amino Acid Biosynthesis in Higher Plants R.A. Jensen, Department of Microbiology and Ceil Science $\$ 207,000$ (2 years)}

The biosynthesis of aromatic amino acids in higher plants is of great significance, not only because of the role of aromatic amino acids in protein synthesis, but because they are precursors of a vast array of compounds of biotechnological and medical interest. Enzyme levels in the pathway have been shown to be regulated in resporise to physiological stage of growth and to stress induced by mechanical wounding. We have elucidated the enzyme steps of biosynthesis and allosteric patterns of control for the pathway located in the chloroplast compartment. A separate enzyme network in thie cytosol has been partially identified and comprehensive enzymological characterizations will be carried out. The post-chorismate portion of the two pathways will be analyzed at the molecular-genetic level. Polyclonal antibodies raised against enzymes purified to homogeneity will be used to clone and obtain the nucleotide sequences of full-length cDNAs. Regulation of aromatic biosynthesis will be characterized at both the levels of enzyme expression and mRNA transcript formation during the growth cycle of suspension cell cultures of Nicotiana silvestris, in specialized tissues of organismal plants, and in response to environmental cues such as light/dark treatment, mechanical wounding, or UV illumination. Regulatory mutants capable of amino acid overproduction will be obtained following mutagenesis of hapisid protoplasts. Data obtained will facilitate interpretations of the physical and evolutionary relationslipi between gene pairs encoding chloroplastic and cytoplasmic isoenzymes. 


\section{Georgia State University Atlanta, GA 30303}

\section{Effect of Growth Temperature on Enzyme Folding A.T. Abdelal, Department of Biology}

$\$ 168,000$ (2 years)

Carbamoylphosphate synthetase from Salmonella typhimurium consists of two unequal subunits with molecular weights of 120,000 and 45,000 daltons. We have recently reported the unexpected finding that the growth temperature influences the kinetic and calorimetric properties of this enzyme. This effect could be a consequence of temperature-modulated folding of the enzyme. This hypothesis will be tested by examining the effect of temperature on refolding of denatured enzyme. Kinetic, calorimetric, and refolding studies will also test our hypothesis that certain ligands may influence the choices among folding pathways of the enzyme.

Direct chromosomal sequencing will be employed to determine the sequence changes in the car $A B$ operon of $S$. typhimurium that result in cold-sensitivity. We have shown that this phenotype is the result of defective enzyme folding. The nature and organization of amino acid residues in defective folding will provide information on the specialized sequences that may direct the folding pathway of carbamoylphosphate synthetase.

Numerous reports indicate that thermal stability of enzymes synthesized by thermophiles is a function of the growth temperature. This effect has been interpreted in terms of expression of duplicate sets of genes at different growth temperatures. We will extend our studies to Thermus acquaticus and Bacillus stearothermophilus to test the alternative hypothesis that the observed effect of growth temperature might be a consequence of a mechanism for thermal adaptation that exploits the effect of temperature on enzyme folding.

\section{University of Georgia Athens, GA 30602}

\section{The Metabolism of Hydrogen by Extremely Thermophilic Bacteria} M.W.W. Adams, Department of Biochemistry

$\$ 173,000$ (2 years)

Extremely thermophilic bacteria are a unique group of microorganisms that have the remarkable property of growing optimally near and above $100^{\circ} \mathrm{C}$. They have been isolated mainly from marine volcanic environments, including deep sea hydrothermal vents. We are growing some of these organisms in large scale culture (500 liters) and are studying their pathways of hydrogen $\left(\mathrm{H}_{2}\right)$ metabolism. From the archaebacterium, Pyrococcus furiosus, which grows optimally at $100^{\circ} \mathrm{C}$ and produces $\mathrm{H}_{2}$ via a fermentative-type metabolism, we have purified a new type of $\mathrm{H}_{2}$-producing, nickelcontaining hydrogenase, and its physiological electron carrier, a ferredoxin. Rubredoxin, pyruvate ferredoxin oxidoreductase, and a unique tungsten-containing enzyme, aldehyde ferredoxin oxidoreductase, have also been purified. The latter two enzymes are thought to couple substrate oxidation to $\mathrm{H}_{2}$ production in a new type of tungsten-dependent glycolytic pathway. A similar pathway for $\mathrm{H}_{2}$ production appears to be present in other extreme thermophiles, including species of Thermococcus and Pyrodictium, and "ES-4". The $P$. furiosus enzymes have optimum temperatures for catalysis above $95^{\circ} \mathrm{C}$, and the redox proteins are stable at $95^{\circ} \mathrm{C}$ for at least 12 hours. 2D-NMR and crystallographic techniques are currently being used to elucidate their mechanisms of extreme 
thermostability. In addition, from the most thermophilic eubacterium currently known, Thermotoga maritima $\left(T_{\max }=90^{\circ} \mathrm{C}\right)$, we have purified a new type of iron-containing hydrogenase, which lacks the catalytic iron-sulfur cluster of mesophilic hydrogenases. Since molecular $\mathrm{H}_{2}$ plays a central role in the commercial production of fuels and numerous chemicals, a long term objective of this research is to assess the utility of extremely thermophilic hydrogenases in industrial energy conversions.

\section{University of Georgia Athens, GA 30602}

\section{The Structure and Functions of Oligosaccharins P. Albersheim, Complex Carbohydrate Research Center}

This project is concerned with the isolation and characterization of oligosaccharins, which are naturally occurring complex carbohydrates that possess biological regulatory activities. We have evidence that oligosaccharins, when released from the complex carbohydrates of cell walls, regulate various biological functions within plants. We are studying the following oligosaccharins. [1] Oligosaccharins isolated from plant cell walls that elicit phytoalexin (antibiotic) accumulation in plant tissues. Research in this area is emphasizing the involvement of fungal endopolygalacturonases (EPGs) and a plantderived inhibitor (PGIP) of the fungal EPGs in the release of elicitor-active oligogalacturonides (DP $=10$ 14) from plant cell walls. We are particularly interested in elucidating the mechanism by which EPGs cause necrosis in some plants. [2] An oligosaccharin that may trigger the hypersensitive resistance response in plants. We have purified to homogeneity an endoxylanase and an arabinosidase secreted by Magnaporthe grisea that are involved in the release of an oligosaccharin from isolated plant cell walls that kills plant cells. At least one other factor (enzyme?) appears to be involved in this process. We are cloning the endoxylanase with the goal of determining genetically whether it is required for eliciting the hypersensitive response to this pathogen. We are purifying and characterizing the bioactive oligosaccharide(s). The same of other wall fragments that kill plant cells are released by autolysis from walls isolated under conditions where the plant's own enzymes are not denatured. We are attempting to identify these fragments. [3] Fungal glucans, when sprayed on Nicotianae, protect the Nicotianae from virus infection. The resistance does not involve any of the well-characterized mechanisms of resistance such as phytoalexin, lignin, callose, or hydroxyproline-rich glycoprotein synthesis, or the accumulation of pathogenesis-related proteins. We are trying to determine the mode of action of the fungal glucans and to identify the glucan oligosaccharide (it is not the phytoalexin elicitor) that activates viral defense. [4] An oligosaccharin derived from xyloglucan that inhibits auxininduced growth. The first oligosaccharin in this series contains nine sugars. Recently, a more active 11-sugar oligosaccharin has been identified. We have also purified to homogeneity and are cloning an $\alpha$-fucosidase of plant cell wall origin that destroys the biological activity of these oligosaccharins. [5] An oligosaccharin that is able to induce the formation of flowers and vegetative shoots and inhibit the formation of roots in tobacco epidermal explants. Our studies with tobacco explants and cells and with soybean cells are emphasizing studies of $\alpha-1,4$-linked oligogalacturonides with DPs of 12-14 that, when released from plant cell walls, by endo- $\alpha-1,4$-polygalacturonase, stimulate floral development and inhibit root development. Oligogalacturonides with DPs of 12-14 also elicit defense responses in plants. Our studies are aimed at elucidating the molecular mechanisms by which oligogalacturonides function. Toward that goal, we are attempting to isolate and characterize a physiological receptor for the oligogalacturonides. We are also investigating the effects of the oligosaccharins on ion transport and membrane polarity of plant cells, which we have some reason to believe are an early step in the signal transduction pathway to the physiological effects of the oligogalacturonides. 


\section{University of Georgia Athens, GA 30602}

\section{The University of Georgia Complex Carbohydrate Research Center (CCRC) - A Department of Energy Unit of the USDA/DOE/NSF Plant Science Centers Program $P$. Albersheim and A. Darvill, Complex Carbohydrate Research Center \\ $\$ 1,264,000$ (FY 90 Funds/15 1/2 months)}

The CCRC, with its multidisciplinary faculty and staff, was formed to serve as a national resource for basic research in complex carbohydrates. The program of this center consists of research, training, and service activities. The CCRC assists scientists from other laboratories in defining the structures and studying the biological functions of plant and microbial carbohydrates. The research of the CCRC focuses on various aspects of carbohydrate science, including methods development, structural characterization and function elucidation. Educational activities involve the training of graduate students, postdoctoral research associates, and visiting scientists in the analytical methods used for studying carbohydrate structures. Two week-long laboratory training courses are held annually for scientists from institutions and industries located throughout the United States. The services offered involve conducting routine analyses of carbohydrate samples provided by scientists from other institutions. These analyses include determination of glycosyl-residue and glycosyl-linkage compositions, and acquisition and interpretation of one-dimensional NMR and FAB-MS spectra. The CCRC also forms collaborations with scientists on more extensive research projects. Collaborations are now in progress with scientists from 25 institutions in the United States and 14 institutions in other countries. These services and collaborative investigations are limited to non-proprietary research. Those interested in assistance, collaboration or training should write to Dr. Russell Carlson, Technical Director, Complex Carbohydrate Research Center, The University of Georgia, 220 Riverbend Road, Athens, GA 30602.

*(A unit of the USDA-DOE-NSF Plant Science Center Program.)

\section{University of Georgia}

Athens, GA 30602

\section{CarbBank - A Structural and Bibliographic Database for Complex Carbohydrates P. Albersheim, Complex Carbohydrate Research Center

CarbBank is a computer program that enables scientists for the first time to systematically and rapidly search for published complex carbohydrate structures and associated text information. Carbohydrate structures linked to literature citations in a computerized database (the Complex Carbohydrate Structural Database, CCSD) (i) allows comparison of newly discovered carbohydrate structures with the structures of those that are known, (ii) brings to scientists an awareness of structural heterogeneity, for example, the attachment of several different oligosaccharides to the same amino acid of a protein, (iii) leads to comparisons of carbohydrate structures between species, and (iv) assists in predicting carbohydrate structures based on limited structural information or properties of known molecules. CarbBank is enhancing the research efficiency and capabilities of many scientists besides those specializing in carbohydrates and is, for the first time, making carbohydrate structures accessible to a wide spectrum of scientists. 
We are continuing to develop CarbBank-associated software, to build the CCSD, and to make the software available as part of larger database efforts administered by the National Center for Biotechnology Information of the National Library of Medicine and by the Chemical Abstracts Service of the American Chemical Society. We intend to create a completely open format for the CCSD and portable, well-documented program modules that will be available to academic and commercial groups interested in creating computer programs that access CCSD data. The CCSD and CarbBank will also be made compatible with several additional computer environments. The CCSD is expected to grow from its present 4,500 records to over 20,000 records. It is our goal to ensure that CarbBank plays a key role in managing carbohydrate structure information in the global biotechnology arena. For information about CarbBank/CCSD, contact: Ms. Dana Smith, CarbBank Database Manager, Complex Carbohydrate Research Center, The University of Georgia, 220 Riverbend Road, Athens, Georgia 30602, Telephone: 404-542-4484, Fax: 404-542-4412.

\section{University of Georgia Athens, GA 30602}

\section{Structural Studies of Complex Carbohydrates of Plant Cell Walls A. Darvill, Complex Carbohydrate Research Center}

The cell walls of a plant determine the plant's structure and morphology and act as a barrier to pests. Cell walls are also a source of complex carbohydrates with biological regulatory properties (oligosaccharins). This project involves the isclation and structural characterization of the complex carbohydrates that constitute approximately $90 \%$ of the primary cell walls of plants. These structural studies emphasize detailed analyses of two pectic polysaccharides, rhamnogalacturonan I (RG-I), and rhamnogalacturonan II (RG-II), and the hemicellulosic polysaccharide xyloglucan. We are chemically and enzymatically cleaving RG-I to produce side chain oligosaccharides, which will be purified and structurally characterized. We are continuing the characterization of the cell wall pectic polysaccharide RG-II by using a combination of chemical and enzymatic methods to determine the order and positions of attachment of the side chains to the $\alpha-1,4$-galacturonosyl backbone with the aim of elucidating the entire glycosyl sequence of this polysaccharide. We are continuing the structural characterization of cell wall xyloglucan by characterizing these endoglucanase-released xyloglucan oligosaccharides that contain 15-20 glycosyl residues. We are generating and characterizing monoclonal antibodies to specific cell wall epitopes for use in localizing polysaccharides and polysaccharide substructures in plant cell walls. The localization studies are a collaboration with Dr. L. A. Staehelin of the University of Colorado and also with Dr. K. Roberts of the John Innes Institute. We are beginning a study on the primary cell walls of Arabidopsis to identify the polysaccharides present and to identify structural changes in these polysaccharides isolated from cell wall mutant tissues supplied to us by Dr. C. Somerville of Michigan State. The research supported in this project will increase our knowledge of the primary structures of cell wall polysaccharides and of their locations in the cell walls and will enable us to build a more complete model of the primary cell wall of plants. Towards that goal, we have formed a collaboration with Roberts, Staehelin, Dr. J. Varner of Washington University, and Dr. D. Lamport of Michigan State to construct a preliminary model of primary cell walls within two years and a definitive model within four years. 


\section{University of Georgia} Athens, GA 30602

\section{Molecular Biology of Lea Genes of Higher Plants \\ L. Dure, Department of Biochemistry}

$\$ 142,992$ (FY 90 Funds/2 years)

The Lea proteins of higher plants are tate embryogenesis abundant. They appear to be universal in occurrence. Certain Lea proteins have been shown to be inducible in non-seed tissue by water stress and/or $A B A$ treatment. Others appear to be seed specific and may function in preventing vivipary, in the acquisition of desiccation tolerance or in other facets of seed formation and survival. Several of the Lea proteins have amino acid sequences that strongly suggest secondary and tertiary structures. Further, these structures in turn suggest functions that would seem important in surviving extreme desiccation, e.g., the mature seed. We wish to describe tolerance to desiccation in terms of the properties of two families of Lea proteins: D-7 and D-29.

This involves biophysical characterization of protein structure by determining molecular weight, Stokes radii, $C D$ spectra, NMR analyses and equilibrium dialysis against specific ions. This requires considerable pure protein. D-7 protein is currently being produced and purified from transformed $E$. coli. We plan to accumulate D-29 via bacterial expression also. Further, we plan the bacterial synthesis of a 39-mer artificial peptide that contains three 11-mer repeating units of amino acid sequence that typifies the D-7 and D-29 proteins and their homologs from other species. The biophysical measurements given above will be obtained for this model peptide. We anticipate that all these measurements will demonstrate a coiled-coil dimer with a very high affinity for $\mathrm{K}+/ \mathrm{Na}+$ and $\mathrm{PO}_{4}{ }^{-}$ ions.

Coincident with these measurements, the most likely 3D structure of the 11 -mer repeating unit will be obtained by computer modeling (program SYBYL) and energy minimization (program $A$.MBER) of a 23-mer abstract peptide containing two 11-mer repeats. From these empirical data and computer derived predictions, we hope to test the idea that the D-7, D-29 families of Lea proteins function as ion carriers in dehydrated cells.

\section{University of Georgia Athens, GA 30602}

\section{Environmental Stress-Mediated Changes in Transcriptional and Translational Regulation of Protein Synthesis in Crop Plants}

J.L. Key and R.T. Nagao, Department of Botany

$\$ 79,000$

The influence of high temperature stress (commonly referenced as heat shock or HS) on mRNA and protein synthesis and on plant growth is the major focus of this research project. Stress agents which mimic somewhat the HS response are used as experimental tools in an attempt to enhance the understanding of regulatory mechanisms operative in the "HS response". The role of one family of general stress proteins in stress sensing and/or stress tolerance of plants (the pCE54 family) and the influence of different common environmental stress agents (e.g., various osmotic stresses) on their synthesis/function is under study.

As a continuing effort to understand the complexity of the HS gene families of soybean and their relationship to those of other organisms, DNA sequence analysis has been completed on a hsp70 gene, a hsp83 gene, and cDNA clones pEV2, pEV6, and pFS2033; these cDNA clones represent a family(s) of hsp mRNAs which are translated on ER-associated polysomes, have a signal sequence which is processed by dog pancreas microsomes (the data on processing accomplished by $E$. 
Vierliny's laboratory), and which represent an endomembrane set of proteins. The specific localization and potential function of these proteins are under study; over-and underexpression of these proteins driven by a strong HS promoter (at least 10- to 20-fold better than their normal promoters) and the physiological consequences thereof represent an initial approach to assess function.

An analysis of the mechanisms operative in self-regulation of the HS response is continuing using runoff transcription analyses, amino acid analogs, cycloheximide, etc., in combination with various HS regimes. Arabidopsis mutant libraries are to be screened for mutants altered in their regulatory responses to HS in order to complement and extend work accomplished to date. Accumulation and stability of HS mRNAs and hsps are an ongoing part of these analyses under both HS and recovery from HS conditions.

\section{University of Georgia Athens, GA 30602}

\section{Regulation of Polyamine Synthesis in Plants}

Polyamines are small positively charged compounds that have been hypothesized to be involved in a wide variety of plant physiological and developmental functions. The regulation of the polyamine synthesis pathway is uniquely interesting because of the existence of two pathways to putrescine synthesis, and the consequent questions of how these two pathways are compartmentalized and how they interact with each other. The specific directions our research is taking are: (1) A molecular characterization of arginine decart oxylase in oats and Arabidopsis using cDNA and genomic DNA clones and a set of antibodies we have developed. This includes analysis of protein processing as part of enzyme activation, cellular localizations, the regulation of arginine decarboxylase by stresses and polyamines, and the construction of transformed plants containing an introduced arginine decarboxylase gene as a means of perturbing the pathway. (2) A similar characterization of the regulation of ornithine decarboxylase using a newly isolated cDNA clone. (3) We are using the clones and antibodies to characterize the polyamine mutant collection we have develop $3 \mathrm{~d}$. Our intent is to understand arginine decarboxylase structure and regulation in detail, and then to further explore regulatory differences between ornithine and arginine decarboxylases.

\section{University of Georgia Athens, GA 30602}

\section{Soybean Ribulose Bisphosphate Carboxylase Small Subunit: Mechanisms and Determinants of RNA Turnover in Higher Plants R.B. Meagher, Department of Genetics \\ $\$ 182,000$ (2. years)}

We have worked on several approaches to understanding the mechanisms and determinants controlling RNA turnover in higher plants during the past year. We concluded our molecular physiological analysis comparing in vitro transcription rates and steady state RNA levels. We have established that a light regulated control mechanism affects soybean ribulose bisphosphate carboxylase small subunit (rbr.S) RNA stability. In a second study, in vivo dimethyl sulfate modification of rRNA and rbcS RNA provided interesting information about RNA structure in light and dark grown leaves. Third, we have completed the initial mapping of the major soybean rbcS RNA degradation products found in total soybean and transgenic petunia leaf RNA. These data allow us to propose three possible models for rbcS RNA degradation, which we are now investigating. We have had success in developing a plant in vitro RNA degradation system, which generates the same rbcS RNA 
products as observed in total RNA. The next years research will focus on characterization and validation of this in vitro system. In the near future we hope to use the system to purify some of the determinants involved in plant RNA turnover.

\section{University of Georgia \\ Athens, GA 30602}

64. Microbiology and Physiology of Anaerobic Fermentations of Cellulose H.D. Peck, Jr., L.G. Ljungdahl, L.E. Mortenson, J.K.W. Wiegel, Departments of Biochemistry and Microbiology

$\$ 315,000$

This project involves the biochemistry and physiology of four major groups (primary, secondary, ancillary and methane bacteria) of anaerobic bacteria, that are involved in the conversion of cellulose to methane or chemirai feedstocks. The primary bacterium, Clostridium thermocellum, has a cellulolytic enzyme system capable of hydrolyzing crystalline cellulose and consists of polypeptide complexes ranging in $M_{r}$ from $\Sigma$ ic 100 million. The complexes attached to the substrate cellulose with the aid of a low molecular (about 1000 daltons) yellow affinity substance (YAS) produced by the bacterium in tr.c presence of cellulose. Properties of the complexes and YAS are studied. Research on the secondary and ancillary bacteria includes acetogens, clor,cridia, methanogens and sulfate reducing bacteria (SRB). Aspects of metabolism are being studied which appear to be relevant for the interactions on consortia and their bioenergetics, particularly related to hydrogen, formate, $\mathrm{CO}$, and $\mathrm{CO}_{2}$. The molecular basis of interspecies $\mathrm{H}_{2}$-transfer and $\mathrm{H}_{2}$-cycling, electron-transfer proteins, ATPase system and enzymes of one-carbon metabolism will receive special focus. Most bacteria appear to produce different proteins with hydrogenase activity which are presumed to be regulated by conditions of growth. Five different hydrogenases have been characterized in detail from the bacteria of interest: The $\mathrm{O}_{2}$-labile $12 \mathrm{Fe}$ bidirectional hydrogenases from $C$. pasteurianium and $A$. woodii; the $\mathrm{O}_{2}$-labile 8Fe uptake hydrogenase from $C$. pasteurianium; the $\mathrm{O}_{2}$-stable $12 \mathrm{Fe}$ hydrogenase from $D$. vulgaris and the $\mathrm{O}_{2}$-stable (NiFe) and (NiFeSe) found in D. vulgaris, other SRB's and the methanogens. The roles of metals in the activation of $\mathrm{H}_{2}$ are being investigated, and genes for the hydrogenases cloned and sequenced to established structural relationships among the hydrogenases. The goals are to understand the roles and regulation of hydrogenases in interspecies $\mathrm{H}_{2}$ transfer, $\mathrm{H}_{2}$ cycling and the generation of a proton gradient. The formate dehydrogenases have characteristics in common with the hydrogenase system: multiple enzyme species with different metal redox centers, MoSeFE, MoFe, WSeFE and pterin; cytoplasmic and periplasmic localizations and involvement in the generation of a proton gradient by vectorial electron transfer. The structures of the metal clusters and their role in the metabolism of formate will be investigated with the goal of understanding the function of formate in the total synthesis of acetate from $\mathrm{CO}_{2}$ and its role in the bioenergetics of these microorganisms. CO dehydrogenase, a key enzyme in the new anaerobic autotrophic $\mathrm{CO}_{2}$ fixation pathway contains Ni plus non-heme iron and the structure of its metal redox centers will continue to be investigated. Additionally, the enzyme studies will be performed using thermophiles and also the isolation of some new pertinent species. The project will also include research on the mechanism of extreme thermophily (growth over $70^{\circ}$ ) in bacteria that grow over a temperature span of $40^{\circ} \mathrm{C}$ or more. These bacteria exhibit a biphasic growth response to temperature and preliminary evidence suggests that the phenomenon is due to the expression of a new set of enzymes. These initial observations will be extended employing techniques of molecular biology. 


\title{
University of Georgia Athens, GA 30602
}

\author{
65. Nitrogen Control of Chloroplast Development and Differentiation \\ G.W. Schmidt, Department of Botany \\ $\$ 156,000$ (FY 90 Funds/2 years)
}

The growth and development of plants and photosynthetic microorganisms is commonly limited by the availability of nitrogen. When the synthesis of amino acids, nucleic acids and pigments is restricted by nitrogen deprivation, these organisms appear to respond by altering the activities of photosynthesis and other metabolic pathways. Hence, the classical symptom for nitrogen deficiency is chlorosis. Our work concerns understanding the mechanisms by which plants and algae that are subjected to nitrogen deprivation alter the composition of photosynthetic membranes and enzymes involved in photosynthetic carbon metabolism. Toward these ends, we study biosynt. vetic and gene expression processes in the unicellular green alga Chlamydomonas reinhardtii which is grown in an ammoniumlimited continuous culture system. We have found that the expression of nuclear genes, including those encoding for light-harvesting proteins, are severely repressed in nitrogen-limited cells whereas, in general, chloroplast protein synthesis is attenuated primarily at the level of mRNA translation. Conversely, nitrogen deprivation appears to lead : = enhanced synthesis of enzymes that are involved in starch and storage lipid deposition. In addition, as a possible means by which photosynthetic electron transport activities and ATP synthesis is sustained during chronic periods of nitrogen deprivation, thylakoid membranes become enriched with components for chlororespiration. Characterization of the chlororespiratory electron transport constituents, including cytochrome complexes and $\operatorname{NADP}(\mathrm{H})$ dehydrogenase is a major current effort. Also, we are striving to isolate the genes encoding chlororespiration proteins toward determining how they and others that are strongly responsive to nutrient availabilit ${ }_{j}$ are regulated.

\section{University of Georgia Athens, GA 30602}

\section{Molecular Characterization of a Maize Regulatiory Gene \\ S.R. Wessler, Botany Department \\ $\$ 178,000$ (FY 90 Funds/2 years)}

The $R$ gene family controls the spatial and temporal distribution of anthocyanin deposition in the maize plant. Prior genetic and molecular analysis indicated that each family member encodes a protein with features of a transcriptional activator including large acidic and basic domains. Part of the basic domain contains a helix-loop-helix motif presumed to be involved in DNA binding and dimerization.

To understand how expression of this gene family results in the observed variety of pigmentation patterns characteristic of different maize strains, we have focused on both the expression of individual $R$ genes and the function of the $R$ protein. During the past year we have shown that the $R$ gene $L c$ is regulated at 3 levels: transcription initiation, post transcripticrial processing and translational. Over the next funding period, we will refine this analysis and investigate the involvement, if any, of each level of control in determining tissue specific pigmentation patterns. Structure-function studies of the $\mathrm{R}$ protein are centered around analysis of a large collection of $D s$-induced mutations (isolated by $\mathrm{J}$. Kermicle, Wisconsin) that display graded effects on pigmentation levels. Finally, we have isolated a $R$ homolog from rice and are using particle bombardment to characterize this gene and maize-rice chimeric constructs with respect to their ability to activate $R$ inducible promoters in both maize and rice backgrounds. 


\section{University of Georgia Athens, GA 30602}

\section{Biochemistry and Genetics of Autotrophy in Methanococcus W.B. Whitman, Department of Microbiology}

$$
\$ 142,000 \text { (2 years) }
$$

Even though methanogenic bacteria catalyze the terminal step in the anaerobic oxidation of organic matter, these bacteria have a very limited catabolic capability. Paradoxically, many methanogens are also autotrophs and utilize carbon dioxide as a major carbon source. To understand the apparent lack of catabolic activity toward complex substrates, two key biosynthetic anabolic enzymes systems, the carbon monoxide dehydrogenase (CODH) system and the pyruvate oxidoreductase/synthase (POR) system, will be characterized. Both of these enzyme systems function catabolically in other bacteria. Using enrichment methods developed in this laboratory, eight acetate auxotrophs of Methanococcus maripaludis have been isolated and shown to be defective in the CODH system. The proposed research will further characterize the $\mathrm{CODH}$ system from these auxotrophs and the wild type to identify the biochemical basis for these mutations. Because some of the auxotrophs are also defective in POR, the apparent coupling of the CODH system and POR will be explored. Preliminary studies have also demonstrated that the methanococci are unable to oxidize pyruvate in the presence of $\mathrm{H}_{2}$. However, in the absence of $\mathrm{H}_{2}$ resting cells will utilize pyruvate as an electron donor for methanogenesis. Genetic and biochemical studies will be performed to further examine the unidirectional nature of this enzyme system under $\mathrm{H}_{2}$ and pyruvate oxidation in vivo. To facilitate genetic studies in the methanogens, a shuttle vector will be constructed from the methanococcal plasmid pURB500, the Escherichia colicloning vehicle pUC19, and the puromycin-resistance cassette provided by Drs. Klein and Possot. If successful, a library of methanococcal chromosomal DNA will be constructed in the shuttle vector and used to complement the acetate auxotrophs. In this way, the genetic basis for these mutants may be determined.

\section{University of Georgia}

\section{Athens, GA 30602}

\section{Hemicellulases from Anaerobic Thermophiles J. Wiegel, Department of Microbiology}

The goal of this research effort is to obtain an anaerobic thermophilic bacterium that efficiently converts various hemicellulose-containing biomass to ethanol over a broad $\mathrm{pH}$ range. The strategy is to modify the outfit and regulation of the rate-limiting hemicellulase(s) in the ethanologenic, anaerobic thermophile Thermoanaerobacter ethanolicus, isolated previously by us. This requires knowledge of the involved enzymes. Thus, for this application the SHORT TERM GOAL is to characterize hemicellulolytic enzymes from this and other anaerobic thermophiles. This will extend the presently limited knowledge of hemicellulases in anaerobic bacteria. The tasks include: i) final characterization (kinetic data with various oligosaccharides) of the purified xylosidase-arabinosidase from $T$. ethanolicus; ii) purification and isolation of thermostable xylanases with a pH optimum in the neutral to slightly alkaline and in the slightly acidic $\mathrm{pH}$ range $(\mathrm{pH} 4-5)$, respectively, from new isolates; iii) purification and isolation of the special xylanase(s) and O-methyl- $\alpha$-glucuronidase from Clostridium thermohydrosulfuricum which effectively degrade 4-O-methyl glucuronic acid substituted oligo-xylans; and iv) the elucidation of regulatory properties of these enzymes. 


\section{University of Georgia Tifton, GA 31793}

\section{Development of Innovative Techniques That May Be Used as Models to Improve Plant Performance \\ W.W. Hanna and G.W. Burton, Department of Agronomy \\ $\$ 42,000$}

The objectives of this project are to: (1) establish the cytoplasmic diversity of germplasm in the weedy subspecies of the primary gene pool and demonstrate its value, (2) identify mechanism(s) for transfer of germplasm from the secondary gene pool to the cultivated species and evaluate the plant breeding potential of this germplasm, and (3) transfer gene(s) controlling apomixis from the tertiary gene pool to cultivated pearl millet for the purpose of producing true-breeding hybrids. Species within the genus Pennisetum are being used as test organisms. The approach uses plants of wild species with different genetic and cytoplasmic backgrounds, ploidy levels, and chromosome numbers crossed and backcrossed with different genotypes of diploid and tetraploid pearl millet. Genes controlling disease resistance (transferred from a wild grassy subspecies) increased palatability and forage intake. The $A^{\prime}$ genome chromosome of Pennisetum purpureum have been the source of germplasm for production of high yielding pearl millet grain hybrid which could result in the development of a new grain crop in the U.S. A number of intsrspecific hybrids among species of the primary, secondary, and tertiary gene pools have forage potential. A 29-chromosome apomictic $\mathrm{BC}_{3}$ pearl millet plant (with one chromosome from the wild species) has been developed. Seed protein and enzyme studies have provided new information on species relationships among the gene pools.

\section{Harvard University Cambridge, MA 02138}

\section{Unraveling Photosystems}

L. Bogorad, Department of Cellular and Developmental Biology

$\$ 67,600$

The objective of this project is to understand how the photosynthetic apparatus works. One approach to idstifying, characterizing and establishing the role of proteins in the photosynthetic apparatus and to understanding the functions of particular amino acid residues and sequences is through studying mutants. Cyanobacteria are relatively simple organisms that carry out the same type of oxygenevolving photosynthesis as chloroplasts of higher green plants but they are more convenient for certain experiments -- particularly those involving genetic manipulation -- because they are prokaryotic microorganisms. We have generated and selected photosynthetic mutants of the cyanobacterium Synechocystis PCC 6803. A number of mutants with altered photosynthetic properties have been isolated. These are transformable to the wild-type by administration of DNA from the normal parental strain; therefore, the gene which is altered in the mutant can be identified by complementation tests. Through a combined program of detailed analyses of photosynthetic parameters and molecular biology, to identify complementing DNA and the nature of the alteration of the protein product of the gene in the mutant, details of the operation of the photosynthetic apparatus should be revealed. These are the current objectives of this program. 


\section{University of Idaho Moscow, ID 83843}

\section{Genetics and Chemistry of Lignin Degradation by Streptomyces \\ D.L. Crawford, Department of Bacteriology and Biochemistry}

Our goal is to define how lignin peroxidases and other extracellular enzymes are involved in lignin degradation by Streptomyces. We have further examined the biochemistry and genetics of lignin degrading enzyme production by strains of Streptomyces. The lignin peroxidase ALip-P3 of S. viridosporus was characterized kinetically and its activity optimized for oxidation of 2,4-dichlorophenol and vanillyl-acetone. Sensitive spectrophotometric assays were developed for monitoring oxidation of these substrates. ALip-P3 reaction chemistry was examined using both spectrophotometric assays and gas chromatography/mass spectroscopy. Results showed that the enzyme oxidizes phenolic lignin substructure models in strong preference to nonphenolic ones. The peroxidase was purified by antibody affinity chromatography, and its $\mathrm{N}$-terminal sequence is now being determined. The sequence will be used to generate oligonucleotide probes for locating lignin peroxidase genes in other actinomycetes. The ALip-P3 gene was cloned in S. lividans in plJ702 and is now being sequenced. The gene is also being used as a probe to isolate lignin peroxidase genes in other actinomycetes. The lignin peroxidase of $S$. viridosporus was also characterized immunologically and shown to be related to peroxidases produced by other lignin-solubilizing Streptomyces, but not to the $\mathrm{H} 8$ lignin peroxidase of Phanerochaete chrysosporium. Studies with lignin peroxidase deficient mutants showed that the lignin peroxidase of $S$. viridosporus is involved in the solubilization of lignin. The data also show that other enzymes are involved, possibly including extracellular esterases. The results have expanded our knowledge of the biochemistry and genetics of actinomycete lignin degradation and have helped us to better define the role of peroxidases in lignin solubilization. Our ultimate aim is to utilize ligninolytic Streptomyces, or recombinant strains derived from them, and/or to utilize their enzymes, for the bioconversion of lignin to useful chemicals.

\section{University of Illinois Chicago, IL 60680}

72. Heavy Metal-Iux Sensor Fusions and Gene Regulation

S. Silver, Department of Microbiology and Immunology

$\$ 204,000$ (2 years)

The project will study gene regulation of the heavy metal resistance determinants of the two large plasmids of Alcaligenes eutrophus strain $\mathrm{CH} 34$, using primarily gene fusions to reporter genes in vivo and direct transcript mapping (both in vivo and in vitro). The resistance determinants to be studied include: (a) $c z c$, cadmium, zinc and cobalt resistance; (b) $c n r$, cobalt and nickel resistance; (c) chr, chromate resistance; and (d) mer, mercury resistance. Copper resistance and arsenic resistance determinants from other plasmids will be included. Each of the systems is inducible. Each has been studied biochemically and has been cloned and sequenced. Using gene fusions to reporter genes, mostly lux (luciferase) but sometimes lacZ (B-galactosidase) or bla (B-lactamase), we will determine whether regulation is positive or negative and identify the trans-acting regulatory genes. The gene fusions (especially with luciferase) obtained for laboratory studies will be subsequently useful as biosensors that can sensitively and specifically detect toxic metal ions in environmental settings and thus function for field measurements of "bio-available" toxic metal levels. 


\section{University of Illinois Urbana, IL 61801}

\section{Genetics of Solvent-Producing Clostridia H.P. Blaschek, Department of Food Science}

$\$ 203,000$ (2 years)

The overall objective of this project relates to the development of genetic systems and methods for the manipulation of the acetone-butanol-ethanol (ABE) fermentation microorganism, Clostridium acetobutylicum. This will involve the construction of a generic Escherichia coli-Clostridium shuttle vector, the development and optimization of a whole-cell electroporation-induced transformation system, the generation of mutants of $C$. acetobutylicum by transposon mutagenesis, cloning of clostridial genes via transposon targeted mutagenesis, and the selection and characterization of cloned cellulase genes from $C$. cellulovorans. The developed genetic systems will then be utilized to return the cloned cellulase genes back into $C$. acetobutylicum. The conversion efficiency and stability of mutant and/or recombinant strains in batch and continuous culture will subsequently be examined. This will involve preparation of radiolabelled cellulose, batch culture growth experiments, continuous culture in solid substrate fermentor, and plasmid stability and copy number determination. The implementation of these objectives will allow for the development of tailor-made strains with improved biotechnological application for the fermentation industry.

\section{University of Illinois}

Urbana, IL 61801-4798

\section{Mechanism and Structure of the Plant Plasma Membrane $\mathrm{Ca}^{2+}$-ATPase D.P. Briskin, Department of Agronomy $\quad \$ 156,000$ (2 years)}

The plasma membrane $\mathrm{Ca}^{2+}$-ATPase couples ATP hydrolysis to the extrusion of $\mathrm{Ca}^{2+}$ at the plasma membrane of plant cells. This process is important for the maintenance of the low cytoplasmic $\mathrm{Ca}^{2+}$ concentration required for the function of this divalent cation as a second messenger in bioregulatory signal transduction. At present, relatively little is known regarding the structure, enzyme reaction mechanism and transport mechanism of this important transport protein. With this project, a research program will be initiated to further characterize the mechanism and structure of the plant plasma membrane $\mathrm{Ca}^{2+}$-ATPase using red beet (Beta vulgaris L.) storage tissue as an experimental system. The objectives of this project will focus on: (1) development of an improved preparation of the $\mathrm{Ca}^{2+}$. ATPase useful for enzymatic/transport studies, (2) characterization of the $\mathrm{Ca}^{2+}$-ATPase catalytic reaction mechanism using steady-state and transient state kinetic methods, (3) structural analysis of the $\mathrm{Ca}^{2+}$-ATPase at the level of essential amino acids, active site sequence and quaternary arrangement of catalytic subunits, and (4) further characterization of the $\mathrm{Ca}^{2+}$-ATPase transport mechanism. If successful, this project will greatly improve our understanding of how the plasma membrane $\mathrm{Ca}^{2+}$-ATPase mediates energy transduction to $\mathrm{Ca}^{2+}$ transport by defining the stepwise mechanism of substrate hydrolysis and its relationship to $\mathrm{Ca}^{2+}$ translocation. Furthermore, this project will provide information that will be important for understanding structure/function relationships for the plasma membrane $\mathrm{Ca}^{2+}$-ATPase and for the future application of molecular approaches to this enzyme system. 


\title{
University of Illinois
}

\author{
Urbana, IL 61801
}

\section{Photosynthesis in Intact Plants}

A.R. Crofts, Department of Physiology and Biophysics \$ \$220,000 (2 years)

The main goal of the proposal is to understand the mechanism of photosynthesis in intact plants. To this end, we have developed instrumentation and methodology for studying photosynthesis in live plants, and under field conditions. However, the project includes a laboratory based program of research to establish in simpler systems the parameters through which observation of photosynthetic electron transport and energy coupling in intact plants can be measured. We have designed and constructed laboratory and portable versions of several instruments, and used these to characterize these reactions. We are studying the partial reactions of the oxygen evolving complex, the two-electron gate of the acceptor side of photosystem II, the intermediate electron transfer chain, including the $b_{6} / f$ complex, and its interactions with the quinone pool, and the cycle around photosystem I, and activation of the ATPase in intact plants, and regulation through the thioredoxin system. We are using site-directed mutagenesis to explore the structure-function relationship in the two-electron gate, with our present emphasis on the Chlamydomomas system. The detailed kinetic, thermodynamic and structural models we have proposed for these reactions will further our investigations and understanding of the factors which limit photosynthetic efficiency under field conditions. A new area of emphasis in the proposal is an investigation of the mechanism of control of photosynthesis in intact systems under steady-state conditions. We propose a mechanism to account for the observation that the efficiency of photosystem II is modulated by the $\mathrm{pH}$ gradient, and propose to test this. We will also explore other potential rate-determining processes which might be expected to play a controlling role under different conditions. We will continue the development of novel instrumentation, and we will continue and extend the collaborations established around the instrumentation developed under the program.

\section{University of Illinois}

\section{Urbana, IL 61801}

\section{Mechanism of Proton Pumping in Bacteriorhodopsin} T.G. Ebrey, Department of Physiology and Biophysics

The purple membrane of Halobacterium halobium probably represents the simplest biological solar energy conversion system. Light absorbed by bacteriorhodopsin, a small protein whose chromophore is retinal, directly leads to the transport of protons across the cell membrane. The resulting chemosmotic potential can be used to make ATP. An additional feature of the purple membrane is its ability to pump protons over a wide variety of salt concentration including in extreme saline environments. This project investigates the relationship between the transport of protons across the membrane and structure and conformation of bacteriorhodopsin. WJe have proposed experiments to study the $\mathrm{pH}$ dependence of proton pumping. Secondly, we are examining the role of divalent cations and the effect of the large surface potential of the purple membrane on the proton pumping function of this membrane using the photocurrents associated with the f'smping process. Finally, we are studying the role of proteinatable amino acids in proton transport. 


\title{
University of Illinois
}

\author{
Urbana, IL 61801
}

\section{Studies on the Escherichia coli Respiratory Chain \\ R.B. Gennis, Department of Biochemistry}

$\$ 207,558$ (FY 90 Funds/2 years)

Our primary interest is to learn how Escherichia coli generates a proton motive force during aerobic respiration. Previously, the cytochrome o complex was identified as a coupling site in the respiratory chain, where electron transfer is coupled to the electrogenic translocation of protons across the cytoplasmic membrane. The enzyme has been purified and this activity has been demonstrated in reconstituted vesicles. The clone of the cyo operon, encoding all the subunits of this terminal oxidase, reveals striking sequence homology between the bacterial ubiquinol oxidase and the well characterized mitochondrial cytochrome $c$ oxidase. We have confirmed this similarity using biophysical techniques, and we have exploited the similarity to design site-directed mutagenesis experiments. Electron spin resonance as well as low temperature Fourier transform infrared spectroscopy have clearly shown that this enzyme contains a heme-copper binuclear center which is virtually identical to the oxygen-reactive center in cytochrome $c$ oxidase. Work on the eukaryotic enzyme implicates histidines within subunit I as heme and copper ligands, and we have altered each of the seven totally conserved histidines to identify which are metal ligands. Two histidines have been assigned as copper $\left(\mathrm{Cu}_{\mathrm{B}}\right)$ ligands, two histidines are clearly axial ligands for the 6-coordinate, low spin heme center, and one histidine has been assigned as the 5 -coordinate, high spin center. A four-helix bundle can be modelled as the catalytic center and serves as a basis for further experiments, including those related to the proton pump activity of this enzyme.

\section{University of Illinois Urbana, IL 61801}

\section{Regulation of Cell Division in Higher Plants}

T. Jacobs, Departmeint of Plant Biology

$\$ 158,000$ (FY 90 Funds/2 years)

Cell division is arguably the most fundamental of all developmental processes. In higher plants, mitotic activity is largely confined to foci of patterned cell divisions called meristems. From these perpetually embryonic tissues arise the plant's essential organs of light capture, support, protection and reproduction. Once an adequate understanding of plant cell mitotic regulation is attained, unprecedented opportunities will ensue for analyzing and genetically controlling diverse aspects of development, including plant architecture, leaf shape, plant height, and root depth. The mitotic cycle in a variety of model eukaryotic systems is under the control of a regulatory network of striking evolutionary conservation. Homologues of the yeast cdc2 gene, its product, p34, and other components of the MPF complex have emerged as ubiquitous mitotic regulators. We have cloned cdc2 like genes from pea and alfalfa. As in other eukaryotic model systems, p34 of pea is a phosphorylated subunit of a high molecular weight complex, which binds the yeast p13 protein and displays histone $\mathrm{H} 1$ kinase activity in vitro. Our primary objective in this study is to gain baseline information about the regulation of this higher plant cell division control complex in non-dividing, differentiated cells as well as in synchronous and asynchronous mitotic cells. We are investigating cdc2 expression and p34 activity at the levels of protein abundance, protein phosphorylation and quaternary associations. 


\section{University of Illinois Urbana, IL 61801}
79. Genetics of the Methanogenic Bacterium, Methanococcus voltae With Attention to Genetic Expression Mechanisms J. Konisky, Department of Microbiology

$$
\$ 130,000 \text { (FY } 90 \text { Funds/2 years) }
$$

The objective of this research program is to study the genetic, physiology and molecular biology of Methanococcus voltae, a marine archaebacterium. This obligate anaerobe produces methane from the reduction of carbon dioxide by hydrogen. For this study we have chosen to study the molecular biology of the gene encoding a membrane associated ATPase which we believe plays an important role in methanogen energetics.

The structural gene encoding the ATPase has been cloned and sequenced, and studies are underway to characterize those properties which control gene expression. These include the identification of DNA-dependent RNA polymerase binding sites, transcription start sites as well as DNA sequences involved in regulating gene activity. In addition, gene expression is being examined under a variety of physiological conditions so as to better define the role of the enzyme in cell growth.

We have recently been successful in introducing DNA into Methanococcus voltae by electroporation. Using this methodology, we have recently initiated studies to clone the genes encoding components of the coenzyme $M$ biosynthetic pathway. Characterization of the molecular biology of coenzyme $M$ production may reveal novel mechanisms of gene expression in this group of important organisms.

\section{University of Illinois Urbana, IL 61801}

80. Genetic and Molecular Studies on Cytoplasmic Male Sterility in Maize J.R. Laughnan and S. Gabay-Laughnan, Department of Plant Biology

$\$ 170,000$ (FY 90 Funds/2 years)

The objective of this project is to determine the basic mechanisms of cytoplasmic male sterility (CMS) in maize and to understand the genetic and molecular bases for both nuclear and cytoplasmic reversions to male fertility. Genetic studies involve attempts to identify cases of insertion of transposable controlling elements into nuclear $\mathrm{cms}-T$ and $\mathrm{cms}-S$ restorer gene sites and, in the case of restorers of $c m s-S$, also a search for cases in which a transposable restorer-of-fertility ( $R f$ ) gene is inserted into a wild-type maize gene. A number of spontaneous $R f$ genes have arisen in inbred nuclear backgrounds and these fall into two classes--functional and nonfunctional. When plants carrying nonfunctional $R f$ genes ( $R f-n f$ ) are crossed as male parents, there is no seed set on the ears. To date, eight independently-occurring spontaneous $R f-n f$ genes have been identified. The allelic relationships between all newly-arisen $R f$ genes is under study. Molecular analysis will be conducted in which the protein profiles of germinating pollen grains from Rf-nf strains will be compared with normal pollen grains from isogenic controls. Rf-nf genes have been found to be functional in hybrid backgrounds and the genetic nature of this hybrid vigor effect is under study. In cms-S plants, a number of newly-arisen $R f$ genes have been shown to be transposable, as has the standard $R f$ gene. Efforts to characterize these transposition events continue. Genetic studies will indicate whether $R f$ elements transpose to a large number of different sites or whether there are preferred sites for insertion. The molecular determination of CMS is in the mitochondrial DNA (mtDNA). We have shown that in cms-S the organization of the mtDNA is controlled by the nucleus of the cell, that significant changes in mtDNA organization occur when one nuclear genotype is substituted for another, and that similar reorganizations of mtDNA accompany cytoplasmic reversion of $\mathrm{cms}-S$ to male fertility. We 
have characterized cytoplasmic revertants from different inbred backgrounds and have found mtDNA alterations common to all reversion events. All revertants analyzed contain rearrangements at the termini of the mitochondrial genome linearized by recombination between S-episome inverted repeats (IR) and IR-homologous sequences present in the main mitochondrial genome. The region adjacent to the integrated IR is designed $R$. The transcriptional profile of this region changes upon cytoplasmic reversion to fertility. Revertants in all nuclear backgrounds lack a $1.39 \mathrm{~kb}$ transcript present in their sterile progenitors. The nuclear restorer gene $R f 3$ restores fertility to $\mathrm{cms}-S$ plants and also affects expression of the $R$ region, while the two spontaneously-arisen restorer genes studied thus far do not. This is the first molecular evidence that these two nuclear restorer genes are functionally different from Rf3. Additional molecular studies are aimed at characterizing the control the nucleus has over the organization of the mitochondrial genome.

\section{University of Illinois}

Urbana, IL 61801

81. Hydrogen-Independent Methanogenic Systems

R.S. Wolfe, Department of Microbiology

$\$ 86,000$

A new approach to the study of methanogenesis concerns the oxidatic n of alcohols by methanogens. This system, recently discovered by $F$. Widdel, provides a hydrogen-independent approach to the generation of electrons, enabling us to fractionate the system so as to by-pass the hydrogenases and their electron carriers. This approach provides another parameter through which to study the biochemistry of methanorzenesis. The deazaflavin, F-420, has been found to be the coenzyme for a secondary alcohol denydrogenase which was purified to homogeneity from Methanogenium thermophilum. Csiis grown on alcohol and $\mathrm{CO}_{2}$ oxidized secondary alcohols to ketones. These cells when exposed to hydrogen reduced ketones or aldehydes to alcohols. In the four strains studied, these reactions were inducible; cells grown only on hydrogen and carbon dioxide did not catalyze these reactions. We are studying the role of methanofuran and other coenzymes of methanogenesis especially in cells grown in the absence of hydrogen (where the source of methyl groups is not by the reduction of carbon dioxide) and are making comparative studies to hydrogen-grown cells.

\section{University of lowa \\ lowa City, IA 52242}

\section{Molecular Biology of Anaerobic Aromatic Biodegradation \\ C.S. Hanwood, Department of Microbiology}

Our laboratory is investigating the molecular basis of anaerobic benzoate and 4-hydroxybenzoate biodegradation by the bacterium Rhodopseudomonas palustris. Aromatic compounds like these are abundant on earth. They occur naturally - mainly as components of the plant polymer lignin - and they also comprise a large proportion of the toxic synthetic organic molecules that are released into the biosphere. Although a great deal of aromatic material accumulates in anaerobic environments, the enzymatic steps taken in the anaerobic degradation of even the simplest aromatic compounds are poorly understood and the molecular genetics of anaerobic aromatic breakdown are almost completely unexplored. We have recently started to analyze a cloned fragment of $R$. palustris DNA which complements a series of mutants blocked in aromatic utilization. We are identifying regions of interest on the clone by transposon mutagenesis, subcloning, and DNA sequencing. Where possible, functions will be assigned to cloned genes based on the ability of the gene products to catalyze known reactions. We will also examine how the expression of selected genes is regulated. The eventual goal is to elucidate the exact sequence of erizymatic steps in the anaerobic benzoate/4hydroxybenzoate pathways and to identify regulatory elements that are required for the synthesis of 
these enzymes. We also plan to determine how aromatic utilization genes are organized physically on the $R$. palustris chromosome. This information will be valuable should it at any point become desirable to transfer these genes to other bacteria with characteristics that may be particularly well suited for specific applications.

\section{Johns Hopkins University Baltimore, MD 21218}

\section{Hydrogen/Sulfur Metabolism in the Hyperthermophilic Archaebacterium R.J. Maier, Department of Biology $\$ 60,303$ (FY 90 Funds/18 months)}

The mechanisms by which hyperthermophilic archaebacteria grow and carry out metabolic functions at elevated temperatures have yet to be determined. The objectives of the work include developing an understanding of the metabolic characteristics of, and the enzymes involved in, hydrogen/sulfur transformation by hyperthermophilic archaebacteria. Efforts are focused primarily on the autotrophic bacterium, Pyrodictium brockii, which has a reported optimum growth temperature $\left(105^{\circ} \mathrm{C}:\right)$ in pure culture. Biochemical ana genetic characterization of enzymes involved in hydrogen/sulfur transformations for these organisms are being pursued. For example, the $\mathrm{H}_{2}$-oxidizing hydrogenase has been purified. Interestingly, the membrane bound form of the enzyme exhibits considerably more tolerance to thermal destruction than the purified de-lipidated enzyme. The electron transport components between $\mathrm{H}_{2}$ and $\mathrm{S}^{\circ}$ are being characterized. These include the $\mathrm{H}_{2}$-activating hydrogenase enzyme, a quinone, a c-type cytochrome, and the S-reducing complex. Comparisons of the biochemical and genetic properties of these electron transport components will be made with mesophilic counterparts. Continued characterization of both purified hydrogenase and the cloned hydrogenase gene will receive a major research effort. The gene encoding a c-type cytochrome is also being cloned and sequenced, for comparison with the predicted amino acid sequences of c-type cytochromes of mesophiles. The long-term goal is to understand the biochemical basis of extreme thermophily.

\section{University of Kentucky Lexington, KY 40546-0091}

84. Photoinhibition of PSII Reaction Centers; Photoactivation, Reconstitution and
Resynthesis of Reaction Center/Water Oxidizing Complex Components
G.M. Cheniae, Department of Agronomy
$\$ 999,000$

Photosystem II (FSII) is a large membrane-bound multi-protein complex that photochemically catalyzes the oxidation of water and the reduction of plastoquinone. Exposure of oxygenic photosynthetic organisms to visible light intensities higher than those to which they are adapted leads to inhibition of electron transfer reactions near the $Q_{A} / Q_{B}$ electron acceptors of $P S I I$, and promotes the disassembly of the entire PSII complex. The susceptibility of PSII to photodamage and disassembly is greatly increased $(\geq 40$-fold) by inhibition/slowing of the forward reactions of the water oxidizing complex. In this case, the photodamages are to reactions on the donor side of the Reaction Center without impairment of charge separation from $P_{680}$, via pheophytin, to the first stable electron acceptor, $Q_{A}$. One early event is the appearance of a $12 \mathrm{G}$-wide signal, centered at $g=2.004$, which persists $(\sim 1 / 2$ spin per PSII) at $4^{\circ} \mathrm{C}$ in subsequent darkness. This presumed carotenoid cation correlates with a quencher of variable Chlorophyll a fluorescence yield. Exogenous reductants $\left(\mathrm{NH}_{2} \mathrm{OH} \geq \mathrm{NH}_{2} \mathrm{NH}_{2}>\mathrm{Mn}^{2+}\right)$ reduce the quencher, but do not reverse other photoinhibition effects. A chlorophyll cation radical 
( 1/2 spin per PSII) also is observed during illumination of photoinhibited membranes, and its extent of rapid decay in subsequent darkness appears to be determined by the redox potential state of cytochrome $b_{559}$. No discernable decoupling of the antenna complex from the Reaction Center is observed; thus, the chlorophyll molecule yielding the cation radical cannot be assigned to a "special" chlorophyll serving to couple antenna pigments to the Reaction Center.

\section{University of Kentucky Lexington, KY 40546-0091}

\section{The Role of Purine Degradation in Methane Blosynthesis and Energy Production in Methanococcus vannielii \\ E. DeMoll, Department of Microbiology and Immunology \\ $\$ 68,000$}

Purine degrading metabolic pathways have been discovered in Methanococcus vannielii. We are currently studying the relationships between purine degradation, methane biosynthesis, and tetrahydromethanopterin (MPT) biochemistry in $M$. vannielii. The purine degrading pathway of $M$. vannielii resembles that described for the clostridia, however in the final steps MPT replaces tetrahydrofolic acid. $M$. vannielii can use certain purines as partial carbon and energy and sole nitrogen source. The 2-, 6-, and 8-carbon atoms of xanthine may all be eventually converted by $M$. vannielii to methane. The 2 - and 6 -carbons enter the methane biosynthetic pathway at the beginning as $\mathrm{CO}_{2}$, however the 8-carbon apparently enters the methane pathway in the form of 5,10-methenylMPT. We are specifically trying to determine exactly how this carbon is converted to methane. Glycine is a product of clostridial purine degradation. Its further metabolism by clostridia generates one mole of ATP. Another goal of our studies is to examine possible further metabolism of glycine by $M$. vannielii. In $M$. vannielii glycine is cleaved to $\mathrm{CO}_{2}$ and ammonia. The fate of the 2-carbon is not known, however we have evidence that it is transferred to MPT forming 5,10-methylene-MPT. We are also examining whether various forms of MPT freely exchange between enzymes of the methane biosynthetic pathway and other enzymes that likely would use one of these forms of MPT, such as thymidylate synthetase, or whether an MPT molecule remains more or less dedicated to the methane biosynthetic pathway. A final objective of our work is to measure the extent to which carbon atoms released during purine degradation appear in biosynthetic and energy producing pathways in $M$. vannielii.

\section{Lawrence Berkeley Laboratory Berkeley, CA 94720}

86. Enzymatic Synthesis of Materials

M.D. Alper, M. Bednarski, H.W. Blanch, D. Clark, J.F. Kirsch, P.G. Schultz, and C.-H. Wong, Center for Advanced Materials $\$ 155,000$

The goal of this research (jointly funded by the Division of Materials Sciences) is the use of natural, engineered and "created" enzymes to synthesize new materials. The unique stereochemical control exerted by enzymes and their ability to catalyze reactions at low temperature will allow the synthesis of materials with structures and therefore properties that cannot be achieved using conventional synthetic routes.

Initial efforts are focused on the design of reaction conditions for the enzymatic synthesis of polymeric materials; engineering of enzyme structure and activity to allow the binding and polymerization of novel monomers; characterization and processing of the polymer products of these reactions and understanding the structure/function relationships of this new class of materials. Work is also progressing on the synthesis of organic thin films of metal and semiconductor surfaces to alter 
adhesive and other interfacial properties and to fabricate sensor devices. Genes have been cloned and expressed to allow production of natural and engineered enzymes. Enzyme thermal stability has been explored. Active sites have been altered through site-directed mutagenesis and other techniques to help understand enzyme/substrate binding and provide a rational basis for modifications required for binding and reaction with unusual substrates. A novel polymer has been synthesized that can be bound to enzymes thus allowing them to retain activity in unbuffered water, high temperature and organic solvents.

\section{Lawrence Berkeley Laboratory Berkeley, CA 94720}

\section{Charaterization of Carotenoid and Bacteriochlorophyll Biosynthesis Genes from a Photosynthetic and a Non-Photosynthetic Bacterium J. Hearst \\ $\$ 290,000$}

Carotenoids are highly colored pigment molecules which protect cells or tissues against photooxidative damage and serve as accessory pigments for harvesting light in photosynthetic systems. Various carotenoids have a wide natural distribution and are found in both photosynthetic and non-photosynthetic organisms from bacteria to plants. Genes required for carotenoid (crt) biosynthesis in Rhodobacter capsulatus, a purple photosynthetic bacterium, were cloned and sequenced in our laboratory. Genes required for yellow pigment production are present on a $12.8 \mathrm{~kb}$ chromosomal fragment in Enwinia herbicola, a phytopathogenic non photosynthetic bacterium. We have isolated the carotenoids produced by both Enwinia herbicola and by Escherichia coli containing this Enwinia crt geise cluster by HPLC. Carotenoid components were characterized by nuclear magnetic resonance and absolute configuration of zeaxanthin diglucoside established by circular dichromism. The levels of carotenoid genes expressed in $E$. coli were about twice as high as the level expressed in Enwinia itself. All of the genes required for carotenoid biosynthesis are present on this $12.8 \mathrm{~kb}$ fragment transformed in E. coli. We have also sequenced this gene cluster from Enwinia herbicola strain Ehu 10 carried on the plasmid PPL376. The sequence suggests the presence of 12 open reading frames (orfs). To determine the minimum size of the plasmid required for full expression of the crt genes, a deletion plasmid pAPU211 has been constructed in pUC19 from the PPL376 plasmid. The carotenoids produced by this construct are brightly yellow colored, like the original plasmid pPL376 and likewise accumulate diglucosylated zeaxanthin. Further deletion and frame shift mutations have been generated, resulting in phenotypes with pink, white, and yellow colors. Carotenoids accumulated by these mutants have been successfully identified and various gene assignments for Erwinia herbicola carotenoid pathway have been established. It is clear that all the genes for carotenoid biosynthesis are clustered together and only six or seven genes are required for synthesis of zeaxanthin diglucoside.

The bchCA operon in the photosynthetic bacterium $R$. capsulatus encodes several genes for bacteriochlorophyll biosynthesis, and its expression is regulated 3 fold by oxygen. In order to study the mechanism of oxygen regulation of transcription, plasmids containing fusions of DNA upstream from the bchCA operon to the lacZ gene from $E$. coli have been constructed. From a deletion analysis, we show that less than $100 \mathrm{bp}$ upstream from the transcriptional start site of the bchCA operon are required to confer oxygen regulation. Vithin this region, there are a number of sequence motifs which have been suggested to be important for regulation, including a s70-like promoter, a palindrome and an AT-rich region. Further deletion indicates that the AT-rich region is required for the anaerobic induction of transcription. Site-directed mutations demonstrate that the s70-like sequence is the functional promoter in $R$. capsulatus and that the palindrome sequence is a binding site for an unknown repressor. The exact roles of the AT-rich region and the palindrome sequence are being further studied. Since both the 570 -like promoter and the palindrome sequence are conserved in many other photosynthesis operons of $R$. capsulatus, the conclusion from this study may be generally 
applicable to the transcriptional regulation of photosynthesis gene expression in this organism. Using a DNA-Affinity column containing the consensus palindrome sequence, a protein has been isolated which exhibits selective binding to this consensus sequence, as well as to the 200 bp region upstream of the bchCA operon which contains an imperfect version of the palindrome (mobility shift assay). The $\mathrm{N}$-terminal amino acid sequence of this binding protein has been obtained, and an oligonucleotide probe has been constructed for the purpose of locating the gene coding for the protein. Further in vitro binding studies are underway to better characterize this and other potential regulatory factors.

We have made great inroads into understanding chlorophyll (Chl) and bacteriochlorophyll (Bchl) syntheses. During the past year, we completed the sequence of all of the genes required for Bchl biosynthesis in $R$. capsulatus. Analysis of these sequences led to the discovery of a new class of proteins, the $\mathrm{Chl} F e$ proteins, which are involved in the ring reductions of $\mathrm{Chl}$ and $\mathrm{Bhl}$ synthesis. These proteins share sequence similarities $(32 \%)$ with the nitrogenase Fe protein, demonstrating that nitrogen fixation and photosynthesis are evolutionarily much more intertwined than had previously been suspected. We have used this similarity to postulate a mechanism of action of the Chi Fe proteins. One of the $\mathrm{Chl}$ Fe proteins in R. capsulatus, bchL, is still more similar (49\%) to the chloroplast gene chIL (formerly fr $\times C$ ) from the nonvascular plant Marchantia polymorpha. Thus we believe we have identified the first sequence of a plant $\mathrm{Chl}$ synthesis gene based on the work initiated in the purple bacteria. As a first step in showing that chlL is involved in Chl synthesis, we generated PCR oligonucleotide primers based on conservation of amino acid seq rences between bchL and chIL and used these to amplify and sequence nearly identical (85-95\%) sequences from cyanobacteria and from the fern chloroplast. Nitrogenase Fe protein, a homodimer containing one [4Fe4S] cluster, channels low potential electrons with the help of ATP hydrolysis to dinitrogenase, an $a_{2} b_{2}$ heterodimer which forms the catalytic portion of the enzyme complex. We postulate that the electrons that reduce chlorophyllide a enter the enzyme complex through the [4Fe4S] cluster, that they are then transferred to the catalytic portion of the complex, and that the energy for the reaction comes from ATP hydrolysis. Additional work is under way to determine the distribution of $\mathrm{Chl} F e$ proteins and to study various aspects of their regulation.

\section{Lawrence Berkeley Laboratory Berkeley, CA 94720}

88. Mechanism of Water Splitting and Oxygen Evolution in Photosynthesis M.P. Klein, Chemical Biodynamics Division

Oxygen evolution in photosystem II of green plants is thought to involve reactions through a cycle of four states by which electrons are removed from two $\mathrm{H}_{2} \mathrm{O}$ and donated to the oxidized P680 reaction conter. The most successful interpretation of the data regarding this cycle is Kok's S-state scheme, which postulates a series of five $\left(\mathrm{S}_{0}-\mathrm{S}_{4}\right)$ states through which electrons are cycled during oxygen evolution. Manganese is thought to play a central role in these reactions. $X$-ray absorption spectroscopy using synchrotron radiation is used to determine the structural and electronic state(s) of the manganese sites. In photosystem II particles of both spinach and the cyanobacterium Synechococcus sp., we have determined that the manganese occur minimally as a binuclear complex with $\mathrm{Mn}-\mathrm{Mn}$ separation of $.2 .7 \AA$. We observe a $\mathrm{Mn} \mathrm{K}$-edge shift of ca $1 \mathrm{eV}$ to higher energy upon advancing from the $S$, to the $S_{2}$ state, implying an oxidation state increase of $\mathrm{Mn}$. Creation of an $S_{0}$-like state produces a K-edge shift in the opposite direction indicating a reduction of $\mathrm{Mn}$ between $\mathrm{S}$, and $\mathrm{S}_{0}$. There is no further oxidation of $\mathrm{Mn}$ upon advancing from the $\mathrm{S}_{2}$ state to the $\mathrm{S}_{3}$ state although the EPR signal characteristic of the $S_{2}$ state disappears. The structures of the Mn complex in the $S_{i}, S_{i}$ and $S_{3}$ states, determined by EXAFS, are similar and well described as pairs of di- $\mu$-oxo binuclear centers with $\mathrm{Mn}-\mathrm{Mn}$ distances of $2.69 \mathrm{~A}$ and $2.79 \mathrm{~A}$ containirig $\mathrm{Mn}$ (III) and $\mathrm{Mn}(\mathrm{IV})$. The distance between at least one of the $\mathrm{Mn}$ in each of these centers is $3.3 \AA$. Recent data of very high quality indicate small differences between the structures in the $S$, and $S_{2}$ states. Polarized EXAFS 
data using oriented PSII membranes shows that the Mn dimers lie in the membrane plane while the 3.3A vector is perpendicular to the membrane plane. Replacement of $\mathrm{Ca}$ by $\mathrm{Sr}$ has permitted us to establish that there is also a $\mathrm{Mn}-\mathrm{Sr}(\mathrm{Ca})$ interaction at $-3.3 \AA$. Mn EXAFS of PSII particles prepared from Synechococcus grown exclusively on $\mathrm{Br}$ suggest that there is one $\mathrm{Br}(\mathrm{Cl})$ at $-2.2 \mathrm{~A}$. Combining these results yields the first tentative structural model of the Mn cluster of the OEC.

\section{Lawrence Berkeley Laboratory Berkeley, Cf 34720}

89. Chemistry of Phycobiliproteins and Phytochrome

H. Rapoport, Chemical Biodynamics Division

$\$ 20,000$

A complete understanding of the chemistry and stereochemistry of phycobiliproteins and of phytochrome is sought to facilitate full understanding of the role of light in regulation of gene expression in green plants. The chemical structures of phycobiliproteins and phytochrome are being determined, including the detailed nature of the covalent attachments of chromophore to protein, by stereospecific synthesis of model chromophores. Chromophore peptides also are being synthesized to ascertain the effect of the peptide on solution conformations and energy transfer.

Knowledge of this mechanism is required for future improvement in plant growth and quality based on genetic engineering at the molecular level.

\section{Lawrence Berkeley Laboratory Berkeley, CA 94720}

\section{Photosynthetic Membrane Structure and Photosynthetic Light Reactions K. Sauer, Chemical Biodynamics Division}

We are investigating the kinetics and energetics of excitation transfer and trapping in antenna complexes and reaction centers of photosynthetic membranes using wavelength-resolved fluorescence decay measurements. This process typically occurs within less than a nanosecond following the absorption of visible light photons. Studies using $X$-ray crystallography from several laboratories have provided structural information for several of these complexes, and this detailed information has enabled us to carry out excitation transfer calculations using exciton theory and/or FÖrster inductive resonance transfer applied to pigment arrays of known geometry. The cyanobacterial pigment $C$-phycocyanin coordinates published based on revised $X$-ray crystallographic structure determinations make it clear that some delocalization should be occurring by the exciton mechanism. As a consequence, there should be some signficant excited state relaxation that is faster than has been resolved in measurements to date, and we will investigate this faster time domain using improved time resolution of our kinetic spectrometer. The availability of cyanobacterial mutants that are deficient in particular components of the phycobilisome antenna complexes has allowed us to investigate the effect of these deletions on the flow of excitation energy in these architecturally complex structures. Reaction center complexes often incorporate or are associated with antenna pigments that have lower energy excited states than that of the charge-separated donor-acceptor state of the reaction center itself. It appears that in these cases there is a thermal activation character to the excitation trapping process that results in a pronounced temperature dependence of competing processes, such as fluorescence. We have extended our studies to a chlorophyll a/b protein complex associated with Photosystem I of higher plants. This complex exhibits pronounced enhancement of long-wavelength fiuciescence at low temperativies even in the absence of reaction centers. We are investigating the role that these low lying excited states play in photosystem architecture and the mechanism of energy conversion. 


\section{Lawrence Berkeley Laboratory Berkeley, CA 94720}

\section{Center for the Analysis of Plant Signal Transduction} S.-H. Kim

This center will address the molecular and biochemical mechanisms by which plants perceive signals and the subsequent signal transduction events and gene expression that occurs in response to such diverse stimuli as light, plant hormones, and plant pathogens. Since this project was initiated in the latter part of FY 90, it is too early to report results.

\section{Lehigh University \\ Bethlehem, PA 18015}

\section{Post-Transcriptional Regulation of Chloroplast Gene Expression by Nuclear Encoded Gene Products \\ M.R. Kuchka, Department of Biology \\ $\$ 162,000$ (FY 90 Funds/2 years)}

The plant cell nucleus encodes gene products which are required for the expression of chloroplast genes. Our goal is to understand the mechanisms by which these nuclear gene products work to affect chloroplast gene expression in a gene- and stage-specific manner. We have focused our attention on the synthesis of a single chloroplast gene product, the D2 protein of Photosystem II. Our approach has been genetic, analyzing nuclear mutants of the unicellular green alga Chlamydomonas reinhardtii which are blocked at specific steps in the expression of D2. We have determined that the products of at least three different nuclear genes are required for D2 expression. One of these nuclear gene products is necessary to stabilize the PsbD mRNA encoding the D2 protein, and at least two are needed to translate the mRNA. We seek to understand at the molecular level the roles of these nuclear gene products in D2 synthesis. To this end, we are characterizing cis- and trans-acting elements that are important in controlling PsbD mRNA stability and promoting translation. Data suggest that sequences other than the 3 ' end of the PsbD mRNA are important in message turnover. We are also studying nuclear gene product involvement in post-transcriptional stages of D2 synthesis by studying $\mathrm{psbD}$ mRNA processing, $\mathrm{psbD}$ message translation in vitro and $\mathrm{psbD}$ mRNA association with ribosomes in mutant cells. Our results show that psbD mRNA is ribosome-bound in these translation mutants, suggesting that later stages of protein synthesis are affected. Suppressors of the original mutations have been identified and are being pursued in order to identify additional genes whose products might interact with the original mutant gene products.

\section{Los Alamos National Laboratory Los Alamos, NM 87545}

93. Carbon Metabolism in Methylotrophic Bacteria

C.J. Unkefer, Isotope and Nuclear Chemistry Division

$\$ 130,000$

Methylotrophic bacteria grow using simple one-carbon compounds such as methane, methanol, or methylamine as their sole source of carbon and energy. The ability to grow on one-carbon compounds gives these organisms considerable industrial potential because they can produce useful substances from inexpensive and renewable precursors. We are using ${ }^{13} \mathrm{C}$-labeled compounds and NMR spectroscopy to examine the metabolic pathways used by these organisms for growth. Results from the studies carried out in this program will provide a foundation for the industrial application of these important bacteria. 
Essential to the growth of methylotrophic bacteria is the oxidation of methanol to formaldehyde which is coupled to the synthesis of ATP, providing the energy to the organism for growth. Methanol dehydrogenase $(\mathrm{MDH})$ which catalyzes the oxidation of methanol does not use nicotinamide or flavin cofactors required by most $\mathrm{c}$ ther dehydrogenases. Instead, MDH contains the novel organic cofactor, pyrroloquinoline quinone ( $\left(\mathrm{P}^{\prime} \mathrm{Q}\right.$ ) that functions as a $2 \mathrm{e}^{-} / 2 \mathrm{H}^{+}$redox carrier accepting electrons from the oxidation of methanol and donating them to the electron transport chain. Using ${ }^{13} \mathrm{C}$-labeled precursors and NMR spectroscopy we have examined the biosynthesis of PQQ in Methylobacterium extorquens $A M 1$. We have shown that $P Q Q$ is derived entirely from the amino acids glutamate and tyrosine and are currently attempting to identify intermediates in the biosynthesis of PQQ.

\section{University of Maryland College Park, MD 20742}

\section{Membrane Topography: Photosystem I and II and Antennae in Phycobilisome- Containing Plants \\ E. Gantt, Botany Department \\ $\$ 74,990$}

The long-term objective of this study is to provide a three-dimensional architectural framework of a photosynthetic membrane. Seven chloroplast proteins were localized in Porphyridium cruentum by immunolabelling with colloidal gold on electron microscope sections of cells grown under red, green, and white light of comparable PFDS. Ribulose bisphosphate carboxylase labeling occurred almost exclusively in the pyrenoid. Labeling for the major apoproteins of photosystem I occurred miostly over the thylakoid region and also over thylakoids passing through the pyrenoid. Labeling for photosystem Il core components (D2 and a Chl-binding core antenna protein), for phycobilisomes (allophycoryanin, and a $91 \mathrm{kD} L_{C M}$ linker) and for ATP synthase ( $B$ subunit) were predominantly present in the thylakoid region but not in the pyrenoid. Red light cells had increased labeling for polypeptides of photosystem II, while photosystem I density decreased, compared to white light cells. Conversely, green light cells had decreased density of photosystem II, while photosystem I density changed little compared with white light-grown cells. In white light cells immunolabeling also provided a quantitative measure of the photosynthetic membrane components. Photosystem I and II per thylakoid area remained constant over a PFD range of $6-280 \mu \mathrm{E} \mathrm{m}^{-2} \mathrm{~s}^{-1}$. However, ATPsynthase per thylakoid area was ten times greater in high light cells than in low light cells. A comparison of the immunogold labeling results with data from spectroscopic methods and from rocket immunoelectrophoresis indicates that this technique can provide a quantitative measure of the relative amounts of membrane proteins as well as their localization in specific organellar compartments. Currently, proteins of photosystem I and II are being directly labeled in situ to determine their spatial relationship.

\section{University of Maryland College Park, MD 20742}

\section{Identifying Calcium Channels and Porters in Plant Membranes H. Sze, Department of Botany}

$\$ 83,220$

Many signal-induced responses; in plant cells are linked to transient changes in cytosolic calcium concentration. The role of $\mathrm{Ca}$ as a second messenger in cells requires regulation of the cytosolic ion level. This can be accomplished by the coordination of passive $\mathrm{Ca}$ fluxes via channels that increase cytosolic $\mathrm{Ca}$, and active $\mathrm{Ca}$ transpori (via pumps or antiporters) that lower cytosolic $\mathrm{Ca}$. The transport mechanisms by which plants achieve this are not understood. The aim of our study is to identify and characterize these $\mathrm{Ca}$ transport proteins by purification and reconstitution. We are currently studying the reaction mechanism and regulation of a Ca-pumping ATPase using membrane vesicles from suspension cultured carrot cells. The molecular mass of a $\mathrm{Ca}$ pump was identified as a 
phosphoprotein of $120 \mathrm{kDa}$. Formation of this phosphoprotein was dependent on $\mathrm{Ca}$, enhanced by $\mathrm{La}$ and inhibited by vanadate. Both calmodulin and acidic phospholipids stimulated $\mathrm{Ca}$ transport characteristic of plasma membrane-type Ca-ATPases. Studies to identify and purify the inositol 1,4,5trisphosphate-sensitive $\mathrm{Ca}$ channel on the vacuolar membrane are underway. These studies will lead to a better understanding of $\mathrm{Ca}$ transport pathways, their regulation and their role in signal transduction.

\section{Massachusetts Institute of Technology Cambridge, MA 02139}

\section{Genetic and Biophysical Analyses of the Photosynthetic Reaction Center D.C. Youvan, Departiment of Chemistry}

The reaction center protein from purple nonsulfur bacteria mediates the efficient transduction of light into chemical energy. The structure of this membrane protein has been determined to atomic resolution; we have developed methods to express genetically modified reaction centers in $R b$. capculatus. Mechanistic models of the light reactions of photosynthesis will be tested through in vitro mutagenesis of the reaction center structural genes, followed by spectroscopic analyses of the genetically altered proteins. This research is aimed at understanding how critical amino acid residues (and peptides) interact to modify the chemistry of the prosthetic groups, for example: (I) in determining whether bacteriochlorophyll (BC) or bacteriopheophytin (BP) is bound at particular sites, (2) in facilitating electron transfer between prosthetic groups either by direct mechanisms or superexchange, (3) in modifying redox or spectral properties of the prosthetic groups, (4) in imparting resistance to certain quinone inhibitors, such as the triazine herbicides, and (5) in directing the path of the photoelectron down only one of two apparent pathways in the reaction center. A principal engineering goal of this research will be to construct "wrong-way" electron transfer mutants. This will be accomplished either by changing the specificity of tetrapyrrole binding sites (BC <-> BP interconversion) or by "swapping" entire helices through the pseudo C2 symmetry axis. Such mutants are important for understanding pigment-protein assembly as well as mechanistic aspects of photochemistry.

\section{University of Massachusetts Amherst, MA 01003}

\section{Cellulose Fermentation by Nitrogen-Fixing Anaerobic Bacteria E. Canale-Parola, Department of Microbiology}

$\$ 189,400$ (2 years)

In anaerobic natural environments cellulose is degraded to methane, carbon dioxide and other products by the combined activities of many diverse microorganisms. These microorganisms eist as communities whose members depend for their survival on a wide range of metabolic interactions. We are simulating processes occurring in natural environments by establishing biologically-defined, stable, heterogeneous bacterial communities (consortia) that we use as in vitro systems for quantitative studies of cellulose degradation, and for the investigation of metabolic interactions among members of ceilulose-degrading populations. For these studies, each consortium, consisting of bacterial isolates from the same environment, is cuitured in a chemically-defined medium, under different environmental conditions, with emphasis on combined nitrogen deprivation. In addition, we are studying the sensory mechanisms that, in natural environments, may enable motile cellulolytic bacteria to migrate toward cellulose. This part of our work includes biochemical characterization of the cellulose chemoreceptor of cellulolytic bacteria. Finally, an important aspect of our research is the investigation of the mechanisms by which multienzyme complexes of anaerobic bacteria catalyze the depolymerization of crystailine celiuiuse and of other piant cell wall polysaccharides. The research will provide 
fundamental information on the physiology and ecology of cellulose-fermenting, $\mathrm{N}_{2}$-fixing bacteria, and on the intricate processes involved in $\mathrm{C}$ and $\mathrm{N}$ cycling in anaerobic environments. Furthermore, the information will be valuable in the development of practical applications, such as the conversion of plant biomass (e.g., agricultural, forestry and municipal wastes) to automotive fuels such as ethanol.

\section{University of Massachusetts Amherst, MA 01003}

\section{Genomic Plasticity and Catabolic Potential of Pseudomonas cepacia T.G. Lessie, Department of Microbiology $\$ \$ 169,860$ (2 years)}

The overall aim of the project is to understand the factors underlying the extraordinary genomic plasticity and adaptability of $P$. cepacia. Our primary focus will be the role of the many insertion sequences in this bacterium in genomic rearrangement and gene activation. Specific aims include i) construction of a physical and partial genetic map of the $P$. cepacia genome showing the distribution of various IS elements and ii) analysis of the influence of different physiological conditions on transpositional activity. We have developed a partial microrestriction map using enzymes such as Pacl, Dral, Afnl, and Xbal, which cleave P. cepacia DNA infrequently. We are using Tn5-751 (which contains a Dral site and confers resistance to kanamycin and trimethoprim) to determine the chromosomal location of various catabolic and biosynthetic genes by transposon mutagenesis. Southern hybridization experiments have suggested that IS elements are clustered in a particular region of the genome. Our working hypothesis is that element transposition is triggered in response to environmental stress and that such clustering might facilitate some type of global regulation. We have inserted a trimethoprim resistance cassette into two of the IS elements for which we have complete nucleotide sequence data. The marked elements are being used to examine the influence of various conditions of environmental stress on transposition. Our long-term goal is to gain insight into the mechanisms balancing genomic constancy and evolutionary change in $P$. cepacia.

\section{Meharry Medical College Nashville, TN 37208}

99. Enzymes of Respiratory Iron Oxidation

R. Blake II, Biochemistry Department

$\$ 155,000$ (FY 90 Funds/2 years)

Aerobic respiration on reduced iron is a principal metabolic activity exhibited by certain chemolithotrophic bacteria that inhabit iron-bearing geological formations exposed to the atmosphere. The aim of this research is to continue the systematic isolation and characterization of the respiratory enzymes expressed by these bacteria when grown at $\mathrm{pH} 1.5$ on soluble ferrous ions. The principal organisms currently under investigation include, but are not limited to, Thiobacillus ferrooxidans, Leptospirillum ferrooxidans, and a moderately thermophilic eubacterium known as strain BC. An in situ electrolytic procedure has been developed whereby large masses of iron-grown bacterial cells may be routinely obtained for these studies. $T$. ferrooxidans contains a respiratory chain that features abundant levels of a blue copper protein called rusticyanin. The amino acid sequence of the rusticyanin from $T$. ferrooxidans has been determined. $L$. ferrooxidans contains a respiratory chain that features abundant levels of an acid-stable a-type cytochrome that is reduced directly by Fe(II). Strain $\mathrm{BC}$ contains a respiratory chain that features abundant levels of a novel yellow chromophore that may represent a new enzyme cofactor unique to aerobic respiration on $\mathrm{Fe}(\mathrm{II})$. A pattern of remarkable diversity in the biochemical mechanisms for bacterial respiratory iron oxidation has begun to emerge from these studies. It is anticipated that this project will provide useful information toward manipulating $T$. ferrocxidans and related organisms for commercial use. 


\section{Michigan Biotechnology Institute Lansing, MI 48909}

100. One Carbon Metabolism in Anaerobic Bacteria: Regulation of Carbon and Electron Flow During Organic Acid Production

J.G. Zeikus and M.K. Jain

$\$ 200,000$ (FY 90 Funds/2 years)

The overall objective of this project is to understand the fundamental biochemical mechanisms that control and regulate carbon and electron flow in anaerobic chemosynthetic bacteria that couple the consumption of single carbon compounds and hydrogen to the production of organic acids and alcohols. Fermentation, enzyme, and electron carrier studies in Butyribacterium methylotrophicum, Anaerobiospirillum succiniciproducens and a syntrophic butyrate degrading co-culture are used as model systems. In B. methylotrophicum the metabolic pathway for conversion of CO into butanol and butyrate has been elucidated. We are continuing our studies with regard to the regulation of alcohol dehydrogenase and hydrogenase. In A. succiniciproducens the mechanism of formate production from pyruvate and $\mathrm{CO}_{2}$ fixation-reduction to succinate are investigated by purification and characterization of key electron carriers and oxidoreductases. Cloning of the PEP carboxykinase gene has begun. In the syntrophic butyrate degrader the biochemical mechanism for formate production is studied in relation to analysis of membrane linked ATP synthesis. These studies may yield information on improvement of anaerobic fermentations for production of organic acids or biomethanation of wastes.

\section{Michigan State University DOE Plant Research Laboratory East Lansing, MI 48824}

101. Molecular Basis of Symbiotic Plant-microbe Interactions

F.J. de Bruijn

$\$ 178,249$

The induction of nitrogen-fixing root- and stem nodules on leguminous plants by soil bacteria belonging to the Rhizobiaceae is a highly evolved, complex process, requiring a fine-tuned interaction between the bacteria and their host. We wish to understand the regulatory signals, between rhizobia and plants, that play a role in the symbiotic control of gene expression in both partners, during nodule establishment and in the mature nodule. Specifically we are characterizing the physiological signals and regulatory circuits which play a role in controlling free-living versus symbiotic expression of rhizobial nitrogen-fixation, assimilation and heme-biosynthetic genes. In addition, we are studying the bacterial-and plant-derived signals which are involved in the nodule-specific expression of plant genes encoding nodulins.

\section{Michigan State University DOE Plant Research Laboratory East Lansing, MI 48824}

\section{Enzymatic Mechanisms and Regulation of Plant Cell Wall Biosynthesis}

D. Delmer

$\$ 206,249$

The goal of this task is to elucidate the mechanism and regulation of synthesis of the plant cell wall with emphasis on the biosynthesis of cellulose and callose. We have recently obtained the first evidence which indicates that higher plants contain a cyclic-di-GMP-dependent cellulose synthase, as evidenced by our finding of two cotton fiber polypeptides of 83 and $48 \mathrm{kD}$ which bind the bacterial cellulose synthase activator c-di-GMP with high affinity and specificity. Both show antigenic relatedness to the c-di-GMP-binding polypeptide of the bacterial synthase, and their level increases markedly during cotton fiber development parallel with the increase in rate of cellulose synthesis in vivo. An 84 kD UDP-glucose-binding polypeptide has also been identified as a possible candidate for 
the catalytic subunit. Current work focuses on purification and sequencing of these polypeptides, isolation of genes which code for them, confirming their role in cellulose synthesis and what changes the polypeptides may undergo during cell breakage which leads to loss of synthase activity in vitro. Studies also continue on the structure and regulation of callose synthase in cotton fibers and on the further characterization of the unique cell walls derived from cells of tomato adapted to growth on the cellulose synthesis inhibitor DCB. Having determined that these walls virtually lack cellulose and xyloglucan, we now concentrate our efforts on determining the structure and interconnections among the other residual wall polymers deposited in the presence of DCB.

\section{Michigan State University DOE Plant Research Laboratory East Lansing, MI 48824}

\section{Molecular Mechanisms That Regulate the Expression of Genes in Plants} P. Green

The steady state level of an mRNA depends both on its rate of synthesis (transcription) and its rate of degradation. Rapidly accumulating data indicate that degradation rates of mRNAs in eukaryotes vary over a wide range and can be regulated by a variety of stimuli. However, in contrast to transcription, the mechanisms that control mRNA stability are largely unknown. A major objective of our work is to identify and characterize the molecular components that control the rates of mRNA degradation in plants (e.g., RNases and the mRNA sequences that they recognize) and determine how they interact. Another goal is to understand how certain components respond to plant growth regulators and to environmental stimuli so as to alter selectively the rates of mRNA degradation. We have found that degradation rates of specific transcripts can be measured directly in stably transformed tobacco cells grown in suspension cultures. This system is presently being used to identify sequences that act as stability or instability determinants within natural plant transcripts or those encoded by reporter genes. To determine if results obtained with cultured cells hold true for regenerated plants, we are also exploring ways to measure rates of mRNA degradation in transgenic tobacco. In addition, we have begun to identify the RNases of Arabidopsis as a first step towards differentiating between the RNases that play a role in mRNA degradation and tnose that have other roles in RNA metabolism.

\section{Michigan State University DOE Plant Research Laboratory East Lansing, MI 48824}

\section{Resistance of Crop Plants to Environmental Stress \\ A.D. Hanson}

Plants have biochemical or metabolic adaptations to environmental stresses, as well as adaptations expressed at higher levels of organization. If biochemical adaptations to stress were better understood, they could be used in crop improvement, via conventional or recombinant DNA technologies. This project aims (1) to identify adaptive biochemical responses of plants to stress, (2) to find the enzymes and genes involved, and (3) to explore the effect on the whole plant of genetically modifying biochemical adaptations. We are working on two topics: betaine accumulation and lactate glycolysis. During water- and salt-stress, certain plants accumulate betaine. Much evidence indicates that betaine acts as a non-toxic cytoplasmic osmolyte during stress. Betaine is synthesized in the chloroplast by a two-step oxidation: choline $\rightarrow$ betaine aldehyde $\rightarrow$ betaine. The second step is catalyzed by a stromal, NAD-linked dehydrogenase. We have isolated CDNA clones for this enzyme (betaine aldehyde dehydrogenase, $B A D H$ ) from spinach and sugar beet, and shown that salinity stress causes the BADH miessage to accumulate. BADH genes are now being introduced into tobacco, a betaine-free species. We have identified the first enzyme of betaine synthesis as a ferredoxin- 
dependent choline monooxygenase (CMO). CMO is currently being purified. Lactate dehydrogenase $(\mathrm{LDH})$ is induced by hypoxia in roots and catalyzes lactate glycolysis. We have isolated LDH cDNA clones from barley, and shown that hypoxia greatly increases LDH mRNA levels. LDH genes are being inserted into tomato root cultures.

\section{Michigan State University DOE Plant Research Laboratory East Lansing, MI 48824}

\section{Action and Synthesis of Plant Hormones H. Kende}

The objective of this project is to gain knowledge on the synthesis and mode of action of the plant hormones ethylene and cytokinin. We have been studying the enzymes that catalyze the synthesis of ethylene from S-adenosyl-L-methionine. 1-Aminocyclopropane-1-carboxylate (ACC) synthase is usually the limiting enzyme in this pathway. It can be induced by a variety of chemicals and environmental conditions, including stress. We have cloned a cDNA encoding ACC synthase (ACCSYN-2) that is different from one (ACCSYN-1) cloned by others. We constructed gene-specific probes for both isoforms and showed that expression of ACCSYN-1 is regulated by stress. The level of mRNA encoding both, ACCSYN-1 and 2, is increased during fruit ripening. We are also investigating the ethylene-forming enzyme (EFE) which, up to now, could not be isolated and characterized. We have isolated a partial CDNA clone encoding EFE in peas and expect to have the full-length sequence shortly. This will permit us to study the regulation of EFE. In support of our experiments on ACC synthase and EFE, we have also isolated mutants of Arabidopsis thaliana which are deficient in ethylene biosynthetic capacity. The role of stress ethylene is being investigated in deepwater rice where low-oxygen stress during flooding induces ethylene biosynthesis. Ethylene, in turn, enhances rapid growth of the plants by increasing their sensitivity to gibberellin (GA). Recent results indicate that this is due to ethylene-mediated reduction in the level of a growth inhibitor, abscisic acid. We have returned to earlier work on the induction of nitrate reductase (NR) by nitrate and cytokinins in Agrostemma githago. We are trying to understand whether cytokinins and nitrate regulate the same or different NR genes. First experiments using CDNA probes for NR indicate that the regulation of NR activity is at the transcriptional level and that the two effectors act independently of each other.

\section{Michigan State University DOE Plant Research Laboratory East Lansing, MI 48824}

\section{Cell Wall Proteins}

$$
\text { D.T.A. Lamport }
$$

This program is an approach to primary cell wall function from the perspective of the wall as a pleiomorphic organelle possessing structural integrity and enzymic autonomy. The structure and function of cell wall glycoproteins in general and the hydroxyproline-rich structural wall protein extensin in particular are the focal points of this research. Thus, our recent isolation of several types of extensin monomer suggests an extensin network involving covalent intermolecular crosslinks. Additional evidence based on isolation of monomeric extensin precursors to the putative covalently linked extensin network, suggests that extensin is a transmural protein. Hence the "warp- weft" hypothesis: a crosslinked extensin network ("weft") of defined porosity is penetrated by a "warp" of cellulose microfibrils creating a true molecular fabric. This simple model based on interpenetrating 
networks is analogous to an "angle-interlock" composite and has a profound implication - it could mechanically couple load-bearing wall polymers thereby distributing stress among the coupled wall components. Is it possible that cell extension, so often described as "biochemically controlled creep", can be explained as a slippage of cellulose microfibrils through the pores of an extensin network?

Current work seeks to test the major premises of the warp-weft hypothesis. Do extensin networks of defined porosity exist? If so, do cellulose microfibrils penetrate the pores? We propose four general lines of enquiry: (1) structural elucidation of extensin crosslink sites and network porosity; (2) enzymic generation of the network in vitro; (3) electron microscopy of extensin monomers, oligomers, and network; and (4) comparative biochemistry of newly isolated extensins from graminaceous monocots (Maize), nongraminaceous monocots (Asparagus), primitive dicots (Sugarbeet), and the gymnosperms (primitive and advanced) Ginkgo, Pseudotsuga, Pinus, Gnetum and Ephedra.

\section{Michigan State University DOE Plant Research Laboratory East Lansing, MI 48824}

\section{Interaction of Nuclear and Organelle Genomes L. Mclntosh}

Our goal is to understand, at a molecular level, the regulation of energy balance in photosynthetic organisms. This work encompasses genetic and biochemical approaches to the elements of the electron transport chains in photosynthesis and respiration. Ultimately, these experiments are aimed at understanding how energy balance is regulated through the interactions of the nucleus, mitochondria and chloroplasts.

A model genetic organism, the unicellular cyanobacterium Synechocystis sp. PCC 6803, is being used in combination with directed mutagenesis to "unravel" electron flow in photosynthetic reaction centers. Genes encoding reaction center proteins have been isolated, sequenced, and their expression is being studied. In parallel, mutations encoding altered primary amino acid sequences are being introduced into these genes and the modified genes are then used to replace their wild-type counterparts in Synechocystis 6803 . The result of these types of experiments are genetically modified strains which are used to understand electron flow in photosynthesis as well as how the photosynthetic reaction centers are assembled.

Plant mitochondria possess two respiratory pathways: a normal cytochrome pathway and an alternative pathway (cyanide-resistant respiration). A major difference between these pathways is that electron flow through the alternative pathway does not contribute to a membrane potential and thus "wastes" energy. The appearance of alternative pathway respiration is dependent upon the developmental stage of the plant, tissue type, environmental factors such as cold stress, and other determinants of plant growth and development. Our immediate goal is understand how the respiratory capacity of higher plants is regulated. We have taken a genetic approach to this problem by first cloning a nuclear gene, aox 1 , which encodes all or part of the alternative terminal oxidase. This gene will be used to alter the expression of the alternati 'e oxidase in plants. A similar approach is being initiated for the nuclear encoded components of the o:her plant terminal oxidase, cytochrome oxidase. 


\title{
Michigan State University DOE Plant Research Laboratory East Lansing, MI 48824
}

\author{
108. Sensory Transduction in Plants
}

K.L. Poff

$\$ 179,450$

The primary objective of this project is to understand the mechanisms for the acquisition of environmental information via light and gravity reception. We are studying the blue light photoreceptor pigment system(s), which control(s) numerous light responses such as phototropism in flowering plants, and in addition, we are studying the mechanism for the perception of gravity in gravitropism. For these studies, we are developing a genetic system with which we can dissect the initial steps in the transduction sequences. Screening procedures have been devised and used to identify mutants of Arabidopsis with altered shoot phototropism, altered shoot gravitropism, and/or altered root gravitropism. Based on these strains, one can conclude that shoot phototropism and shoot gravitropism share many common elements, but that shoot gravitropism and root gravitropism are substantially separate pathways. The shoot photo-minus, gravi-normal phenotype should represent an alteration early in the phototropism pathway and could arise from an altered photoreceptor pigment. High resolution analysis of the fluence response relationship for phototropism shows that at least two different photoreceptor pigments are involved. In addition we have identified a strain with its threshold fluence for phototropism increased by a factor of 50. The fluence response relationship for this strain shows that the threshold fluence for one of the photoreceptor pigments has been altered. Based on these data, this strain represents a probable candidate as a photoreceptor pigment mutant. Under conditions of long term irradiation, the plant has the capacity to adapt to the irradiation, such that its responses at the beginning and end of a long irradiation are not equal. An analysis of adaptation indicates that it consists of desensitization, recovery and enhancement. Desensitization is under strict blue light control while enhancement is mediated by phytochrome. It is now clear that an understanding of adaptation is necessary to understand the plant's response to long term irradiations. Our approach to the study of the pathways for phototropism and gravitropism includes mutant isolation, and genetic, physiological and biophysical characterization. This genetic/biophysical approach should permit positive identification of the receptors, access into the transduction sequence, and eventual understanding at the molecular level of the events from reception to the bending responses.

\section{Michigan State University DOE Plant Research Laboratory East Lansing, MI 48824}

109. Molecular Mechanisms of Trafficking in the Plant Cell

N.V. Raikhel

Maintenance of separate subcellular compartments in eukaryotic cells depends on the correct sorting and targeting of newly synthesized proteins. Thus, mechanisms must exist in the cell to assure that these proteins are targeted to, and subsequently translocated across, the correct intracellular membranes. We are working with proteins destined for different compartments: vacuoles, cell wall and nucleus. There is increasing evidence that proteins destined for subcellular organelles contain a "sorting signal" which dictates the compartment to which the protein is targeted. We are interested in understanding the molecular determinants of differential protein compartmentalization and in identifying the components of the molecular machinery which carry out the sorting process. Our goals include the analysis and characterization of protein sequences responsible for sorting to the vacuole and to the nucleus and the identification and isolation of one or more putative receptors which recognize these sorting sequences and subsequently mediate protein transport to vacuoles and to nucleus. 
Plants possess a large arsenal of both constitutive and inducible biochemical and mechanical defense mechanisms against various potential fungal pathogens and insect pests. Several arguments support the hypothesis that chitin-binding proteins are involved in host-defense: 1) these lectins exhibit high binding affinity for poly- $\mathrm{N}$-acetylglucosamine (chitin), which is a major component of the fungal cell wall and the peritrophic membrane of the insect midgut; 2) in in vitro experiments chitin-binding proteins show insecticidal and antifungal activities; 3 ) chitin-binding proteins are predominantly located in the protective peripheral tissues of the organs and almost all of them are wound-inducible. By studying molecular mechanisms of regulation and expression of chitin-binding genes we would like to understand the endogenous function of the proteins they encode.

\section{Michigan State University DOE Plant Research Laboratory East Lansing, MI 48824}

\section{Physiological and Molecular Genetics of Arabidopsis \\ C.R. Somerville}

$\$ 201,550$

The overall objective of this task is to develop genetic methods for the analysis and modification of specific physiological processes in plants. Our research is primarily concerned with a genetic analysis of membrane lipid composition in Arabidopsis. We have identified a series of mutants of Arabidopsis in which the fatty acid composition of leaf and seed lipids has been altered by specific deficiencies in fatty acid desaturases, G3P acyltransferase or elongases. Detailed analysis of lipid metabolism in the mutants has provided new insights into the regulation of membrane lipid acyl group composition. The ability of the mutants to compensate, in several cases, for the loss of specific enzyme activities suggests that the composition of membranes is at least partially regulated in response to functional properties of the membranes. The mutants have also been useful for examining the roles of lipid composition in determining membrane structure and function. In general, it appears that under growth conditions in the laboratory, large changes in lipid acyl group composition have relatively slight effects on the function of membrane associated processes such as photosynthetic electron transport but may have significant effects on the overall organization of the membranes. We are currently exploring ways of exploiting the availability of the mutants to clone the genes affected.

\section{Michigan State University DOE Plant Research Laboratory Eas: Lansing, MI 48824}

\section{Molecular Basis of Disease Resistance in Barley S.C. Somerville}

We have chosen to work with two complementary host-pathogen systems. One is the powdery mildew disease of barley caused by the fungal pathogen Erysiphe graminis $\mathrm{f}$. $\mathrm{sp}$. hordei and the other is the interactions between Arabidopsis thaliana and the bacterial pathogens Xanthomonas campestris pv. campestris and Pseudomonas syringae pv. syringae. For the barley powdery mildew disease, our specific objective is to identify the gene(s) at the barley Ml-a resistance locus. The characterization of an Ml-a gene product will be an important step in determining a key biochemical component of disease resistance. In addition, understanding the genetic mechanism that is responsible for the highly polymorphic nature of the $\mathrm{MI}$-a locus is of both practical and theoretical interest, since the genetic structure of this locus will dictate the range of novel resistance alleles that may be created in vitro for the purposes of genetically engineering disease resistance. Arabidopsis is a model plant species that offers many technical advantages for isolating genes, such as disease resistance genes, for which 
biochemical information is lacking. A second objective of our research program is to clone those genes of Arabidopsis that mediate resistance to $X$. c. campestris, the causal agent of the black rot disease, and those genes that regulate responses to non-host pathogens like $P$. s. syringae. These genes will then be used as tools in characterizing host resistance mechanisms.

\section{Michigan State University DOE Plant Research Laboratory East Lansing, MI 48824}

\section{Biochemical and Molecular Aspects of Plant Pathogenesis J.D. Walton}

The objective of this project is to understand the biochemical events that are important to the interaction between fungal plant pathogens and their host plants. We are studying examples of factors that control specificity as well as factors that are necessary for basic compatibility. As our model organism we are using Cochliobolus (Helminthosporium) carbonum, which causes leaf spot disease of maize. C. carbonum can be transformed with exogenous DNA and particular genes disrupted. It also has a sexual stage with two mating types. Race 1 of $C$. carbonum is highly virulent on maize that is homozygous recessive at the nuclear $\mathrm{Hm}$ locus due to its ability to produce $\mathrm{HC}$-toxin, a hostselective toxin. We have cloned the Tox2 gene cluster that controls $\mathrm{HC}$-toxin production and are studying its structure and relation to the enzymology of $\mathrm{HC}$-toxin biosynthesis. In order to study the specificity of $\mathrm{HC}$-toxin against maize we have prepared radiolabelled $\mathrm{HC}$-toxin. Maize leaves can metabolize $\mathrm{HC}$-toxin to the inactive 8-alcohol. Susceptible plants appear to be lacking the enzyme, and NADPH-dependent reductase, that catalyzes this reaction. As examples of basic compatibility factors we are studying cell wall-degrading enzymes made by $C$. carbonum. These enzymes have been proposed, but never shown, to have an important role in penetration, nutrient assimilation, and tissue invasion, as well as in triggering host defenses. We have cloned the single endopolygalacturonase gene and used it to make a mutant of $C$. carbonum with a disrupted polvgalacturonase gene. The mutant was as pathogenic as the wild-type fungus. We have also isolated xylanase and laminarinase and are cloning the corresponding genes.

\section{Michigan State University DOE Plant Research Laboratory East Lansing, Ml 48824}

\section{Developmental Biology of Nitrogen-Fixing Cyanobacteria C.P. Wolk}

Anabaena and related cyanobacteria utilize solar energy to fix nitrogen gas within cells called heterocysts. We have developed a novel system for elucidation of the genetic regulation underlying heterocyst development. We used a derivative of transposon Tn $\underline{5}$ to generate transcriptional fusions of promoterless bacterial luciferase genes, $I U \times A B$, to the Anabaena genome. Genes that responded to removal of fixed nitrogen or to other environmental shifts by altered rates of transcription were identified by monitoring the luminescence of many transposition-derived colonies. Additionally, the lux genes were put under the control of the promoter of a specific gene, the resulting strain was transposon-mutagenized, and many resulting colonies were screened to identify regulatory mutants in which luminescence was prevented or rendered constitutive. Imaging of luminescence by photon-counting microscopy identified the cells in which a particular gene was active. The activity of the promoter of a weakly transcribed gene was first amplified by fusing the gene transcriptionally to the gene for T7 RNA polymerase, and by putting luXAB in a multicopy plasmid under the control of a promoter recognized by that polymerase. We are developing techniques to analyze the physiology of nitrogen-fixing cyanobacteria. This work will facilitate understanding of cellular differentiation and of biological conversion of solar energy. 


\title{
Michigan State University DOE Plant Research Laboratory East Lansing, Ml 48824
}

\author{
114. Environmental Control of Plant Development and Its Relation to Plant Hormones \\ J.A.D. Zeevaart

Plant growth and development are affected by environmental factors such as daylength, temperature, and moisture. Hormones often function as chemical messengers between the perception of an environmental factor and the morphological expression. The objective of this project is to characterize the hormones involved, how their synthesis and catabolism are regulated by the environment, and how these hormones act. In the long-day rasette plant spinach, 3B-hydroxylation of gibberellins (GAs) is required for induction of stem growth. Of the $G A s$ native to spinach, $G A_{1}(3,13$-hydroxylated) is the most abundant bioactive $\mathrm{GA}$. In Arabidopsis, both $\mathrm{GA}_{1}$ and $\mathrm{GA}_{4}$ (3-hydroxylated) are endogenous $\mathrm{GAs}$ and both are active. Since $\mathrm{GA}_{4}$ is converted to $\mathrm{GA}_{1}$, the possibility cannot be ruled out that 13hydroxylation is also necessary for activity.

Studies on the biosynthesis of the hormone abscisic acid (ABA) have demonstrated that three of the four oxygen atoms are derived from molecular oxygen. In stressed leaves incubated in ${ }^{18} \mathrm{O}_{2}$, one ${ }^{18} \mathrm{O}$ atom is rapidly incorporated in the carboxyl group, whereas isotopic enrichment is much less in the oxygen atoms of the ring. This indicates that $A B A$ is derived by oxidative cleavage from a larger precursor (a xanthophyll) with the ring oxygens already present. Apple fruits, unlike water-stressed leaves, synthesize the majority of $\left[{ }^{18}\right.$ O]ABA with the label in the 1 '-hydroxyl position and unlabeled in the carboxyl group. The ${ }^{18} \mathrm{O}$-labeling of the xanthophylls closely parallels that of $A B A$ in the same tissues. Furthermore, the ABA-deficient aba mutant of Arabidopsis is deficient in the epoxycarotenoids, violaxanthin and neoxanthin, and accumulates the biosynthetic precursor zeaxanthin. Thus, the evidence is now conclusive that in higher plants $A B A$ is a breakdown product of xanthophylls.

\section{Michigan State University East Lansing, MI 48824}

\section{Structure of Surface Glycoconjugates of Rhizobium Species and Their Function in Nitrogen Fixation \\ R.I. Hollingsworth, Department of Biochemistry

In our laboratory we are trying to determine what structural features of the LPS or LPS-associated glycoconjugates on the surface of Rhizobium may be conserved between strains of any given species of this genus. This is important for understandirig the specificity of the relationship between the plant host and bacterial symbiont. Purified bacterial surface glycoconjugates are partially hydrolysed to generate fragments which are used in NMR analyses. The structures of the fragments are also studied using classical analytical techniques. The oligosaccharides are conjugated to a protein carrier and used to raise antibodies in rabbits. A fluorescent-labeled goat antirabbit antibody is used to locate rabbit antibodies on cells of a wide cross section of strains following incubation with these rabbit antibodies. This indirect immunofluorescence assay will be applied to a wide variety of strains of any one species and so identify structural features which are species specific. It will also allow us to follow the dynamics of the chemistry of the bacterial cell surface as it is influenced by external factors. This study will be extended to include bacterial strains in which the nodulation genes have been induced. These results will answer the question of the fate of the bacterial cell surface and the LPS structure on nod gene induction. 


\title{
Michigan State University East Lansing, MI 48823-1312
}

\author{
116. Role of Acyl Carrier Protein Isoforms in Plant Lipid Metabolism \\ J.B. Ohlrogge, Department of Botany \& Plant Pathology
}

$\$ 82,000$

Our long term goal is to understand how plants control the activity of the fatty acid synthesis (FAS) pathway and how its products are channeled into their diverse roles and locations within the plan!t cell. Acyl carrier protein (ACP) is the central cofactor required for at least 10 reactions of plastid fatty acid metabolism. We have recently described the occurrence and tissue specific expression of two isoforms of ACP at both the protein and mRNA expression level. Preliminary evidence based on in vitro assays indicates that the two forms of ACP have different activity in reactions which direct the distribution of acyl chains within the plant cell.

The general objective of this project is to further examine the biochemical significance of ACP isoforms to plant lipid metabolism. Specifically, we are a) examining the distribution of ACP forms in a variety of photosynthetic species, in various tissues and under different environmental influence; $b$ ) inhibiting the expression of specific isoforms in Arabidopsis using antisense RNA; c) examining the amount and type of acyl groups esterified to ACP which can reflect the status of the fatty acid biosynthetic pathway. We have found that approximately 20 percent of both ACP-I and ACP-II contain long chain acyl groups in actively growing spinach leaves. In addition we have developed methods to examine the composition of the short chain acyl-ACP pool in vivo. The relative levels of ACP-SH, acetyl-ACP and malonyl-ACP are being examined in developing seeds in order to evaluate potential rate limiting steps in plant fatty acid biosynthesis.

\section{Michigan State University East Lansing, MI 48824-1101}

\section{Physiology and Molecular Biology of Extracellular Peroxidases and $\mathrm{H}_{2} \mathrm{O}_{2}$-Generating System of Phanerochaete chrysosporium \\ C.A. Reddy, Dept. of Microbiology and Public Health $\$ \$ 149,500$ (FY 10 Funds/2 years)}

The iignin-degrading basidiomycete, $P$. chrysosporium, produces during secondaly metabolism two families of extracellular heme proteins designated lignin peroxidases (LIPS) and manganese-dependent peroxidases (MNPS), and hydrogen peroxide-generating enzymes (as exemplified by glucose oxidase and glyoxalate oxidase) as major components of its lignin degrading enzyme system. Understanding the physiology and molecular biology of the above enzymes in relation to ligin degradation and degradation of various chloroaromatic compounds by this organism is a major orjective of this project. We have in the past isolated and sequenced several of the major LIP genes of this organism and have reported on a DNA transformation system utilizing an extrachromosomally maintained transformation vector. Detailed mechanistic studies on this novel transformation system are being continued. Using DNA probes specific for each of the major LIP genes, we will study regulation of expression of the individual genes in response to various physiological and environmental parameters which are known to affect lignin degradation by this organism. We have recently isolated various classes of mutants which are defective in one or more of the key enzymes mentioned above. These mutants will be utilized to determine the relative importance of various enzymes for lignin degradation as well as xenobiotic degradation by this organism. We will also study selected groups of wood decay fungi and determine the presence of LIP and MNP genes homologous to those of $P$. chrysosporium. Investigations on the der8-5 mutant, whıch is nitrogen-dereguiated for LiF production, wiil contiinue io determine the nature of carbon and $\mathrm{Mn}(\mathrm{II})$ regulation on LIP and MNP production in this organism. 


\section{University of Minnesota Minneapolis, MN 55455}

\section{The Biochemistry and Enzymology of Halomethyl Corrinoids}

H.P.C. Hogenkamp, Department of Biochemistry

In collaboration with Professor R.K. Thauer of the Philipps Universität, Marburg, Germany, we have investigated the reductive dehalogen ation of carbontetrachloride, trichlorofluoromethane (Freon 11), dichlorodifluoromethane (Freon 12), ch. -otrifluoromethane (Freon 13) and tetrafluoromethane (Freon 14), catalyzed by corrinoids. Corrinoids such as aquocobalamin, cyanoaquocobinamides and even fluoromethylcobalamins, were found to catalyze the reductive dehalogenation of $\mathrm{CCl}_{4}, \mathrm{CFCl}_{3}, \mathrm{CF}_{2} \mathrm{Cl}_{2}$ and $\mathrm{CF}_{3} \mathrm{Cl}$ to carbon monoxide. Freon 14 was not reduced. The rates of dehalogenation decreased in the series $\mathrm{CFCl}_{3}, \mathrm{CF}_{2} \mathrm{Cl}_{2}$ and $\mathrm{CF}_{3} \mathrm{Cl}$. The reductive dehalogenation of $\mathrm{CFCl}_{3}$ by $\mathrm{Ti}(\mathrm{III})$ citrate and aquocobalamin as the catalyst gave, in addition to $\mathrm{CO}(67 \%), \mathrm{CHFCl}_{2}(<10 \%)$, formate $(<5 \%)$ and smaller amounts of $\mathrm{CH}_{2} \mathrm{FCl}, \mathrm{CH}_{3} \mathrm{~F}, \mathrm{C}_{2} \mathrm{~F}_{2} \mathrm{Cl}_{2}$ and $\mathrm{C}_{2} \mathrm{~F}_{2} \mathrm{Cl}_{4}$. The rate and extent of fluoride release was identical to that of the sum of $\mathrm{CO}$ and formate formation. Our results are in accord with a mechanism of dehalogenation, which involves the intermediacy of dihalocarbenes. These dihalocarbenes either hydrolyze to $\mathrm{CO}$ or couple to yield tetrahaloethylenes.

The formation of these highly reactive carbenes from $\mathrm{CCl}_{4}$ and the Freons 11,12 and 13, may explain why these compounds are highly toxic for some anaerobic bacteria. Our results are also in accord with the findings of Egli and coworkers, that some anaerobic bacteria are able to degrade the $\mathrm{CCl}_{4}$ and $\mathrm{CHCl}_{3}$ to $\mathrm{CH}_{2} \mathrm{Cl}_{2}, \mathrm{CH}_{3} \mathrm{Cl}$ and $\mathrm{CO}_{2}$. Carbon monoxide formed from the dihalocarbene intermediates would be oxidized to $\mathrm{CO}_{2}$ via the very active $\mathrm{CO}$ dehydrogenase present in anaerobic bacteria.

\section{University of Minnesota Minneapolis, MN 55455-0312}

119. The Mechanism of Switching from an Acidogenic to a Butanol-Acetone Fermentation by
Clostridium acetobutylicum
P. Rogers, Department of Microbiology $\$ 178,000$ (2 years)

The objective of this project is to elucidate the detailed mechanism by which the solvent-forming bacterium, Clostridium acetobutylicum, regulates the shift in fermentation metabolism between butyric and acetic acid formation during exponential growth and butanol-acetone production triggered by accumulation of butyric acid. Experiments are designed to identify and describe the regulatory genes and protein elements that govern the rate of synthesis of solvent-pathway enzymes. How this regulatory system interacts with induction of sporulation and related formation of intra-cellular granulose or other cell changes will also be investigated.

This research is centered upon the technique of employing transposable elements that create gene fusions and mutations due tu insertion in the chromosome of gram positive bacteria. Our approach is based on recent demonstration in our laboratory and by others of transconjugation of transposon Tn916 into C. acetobutylicum and its insertion into the chromosome. A panel of strains with Tn916 inserts that are also solvent-negative and/or asporogenic will be used to identify specific regulatory genes. A second approach is based upon electroporative transformation of plasmid PTV1 DNA carrying transposon Tn917 into C. acetobutylicum. Insertion of Tn917 lac to report activity of genes and functions in vegetative and stationary or slow-growing cells will be investigated. 


\section{University of Minnesota Navarre, MN 55392}

\section{Genetics of Bacteria that Utilize One-Carbon Compounds}

R.S. Hanson, Gray Freshwater Biological Institute

$\$ 162,000$ (2 years)

Methylotrophic bacteria are those bacteria that grow on one-carbon compounds including methane, methanol, methylamines, some halomethanes, and a few other compounds that do not contain C-C bonds. Those bacteria that grow on methane (methanotrophs) contain particulate methane monooxygenases (MMO's). Some also synthesize a soluble MMO (SMMO) when grown with limiting amounts of cooper. Soluble MMO's oxidize a wide variety of substrates including halogenated low molecular weight hydrocarbons which are not oxidized by pMMO's. All synthesize methanol dehydrogenases (MDH's). SMMO's and MDH's each may comprise $15-20 \%$ of the cellular protein. $\mathrm{MDH}$ synthesis is inducible in some facultative methylotrophs.

We have cloned genes encoding SMMO components and we have mapped several genes required for the synthesis of active MDH. We have identified regulatory genes and a protein that binds specifically to sequences located upstream of the MDH structural gene. The role of this protein in the regulation of $\mathrm{MDH}$ synthesis will be examined using in vitro transcription/translation assays and genetic approaches.

Regulatory sequences that regulate expression of genes that encode the sMMO components will be identified and sequenced. The mechanisms of copper regulated expression will be examined.

\section{University of Missouri Columbia, MO 65211}

\section{Molecular Analyses of Nuclear-Cytoplasmic Interactions Affecting Plant Growth and Yield \\ K.J. Newton, Division of Biological Sciences \\ $\$ 178,957$ (2 years)}

The biogenesis and functioning of mitochondria depends on the expression of both mitochondrial and nuclear genes. One approach to investigating the role of nuclear-mitochondrial cooperation in plant growth and development is to identify combinations of nuclear and mitochondrial genomes that result in altered but sublethal phenotypes. Plants carrying certain maize nuclear genotypes in combination with cytoplasmic genomes from certain teosintes (wild relatives of maize) can exhibit "incompatible" phenotypes, such as reduced plant growth and yield and cytoplasmic male sterility, as well as altered mitochondrial gene expression. The characterization of these nuclear-cytoplasmic interactions is the focus of this project. We are investigating the effects of two maize nuclear genes, $R c \mathrm{~cm} 1$ and $\mathrm{Mct}$, on mitochondrial function and gene expression. We will attempt to clone and characterize each of these genes and to analyze how the products of recessive and dominant alleles at each locus coordinate with products of the teosinte mitochondrial genes to give aberrant plant phenotypes. 


\section{University of Missouri Columbia, MO 65211}

122. Genetics of the Sulfate-Reducing Bacteria J.D. Wall and B.J. Rapp-Giles, Biochemistry Department

The genetics of the sulfate-reducing bacteria are being pursued with efforts focussed on the Desulfovibrio desulfuricans. A small endogenous plasmid, $\mathrm{pBG1}$, identified in $D$. desulfuricans strain G100A has been completely sequenced. It is apparently not stably replicated in $E$. coli as kanamycin resistant transformants are not obtained when a $\mathrm{Kn}^{R}$ cassette is introduced into PGB1. In addition, it is not able to rescue a $\mathrm{PUC} / \mathrm{pBG} 1$ cointegrate at high temperature in a polA temperature sensitive background. The mobilization locus from the InCQ plasmid RSF1010 has been introduced to the cointegrate to allow conjugation into the sulfate-reducing bacteria. The characterizations of this construct are under study.

A 2000 member cosmid library of the DNA of the G100A strain has beer generated and is presently being characterized. The construction of this library was dependent on the development of a satisfactory protocol for the consistent isolation of large pieces of DNA from these bacteria. The presence of significant nuclease activity probably accounts for the historical difficulty in obtaining native plasmids from these bacteria.

We continue to find that $D$. desulfuricans serves as a good expression system for genes from other sulfate-reducing bacteria.

\section{Mount Sinai School of Medicine New York, NY 10029}

\section{The Respiratory Chain of Alkaliphilic Bacteria \\ T.A. Krulwich, Department of Biochemistry}

The long term goals of this project are: (a) to elucidate the spe cial relationship between specific respiratory chain components of the alkaliphile respiratory chain and the ability of this organism to carry out oxidative phosphorylation using a $\mathrm{H}+$-translocating ATPase at low protonmotive force; and (b) to study the basis for the very high cytochrome content of alkaliphile membranes and the qualitative changes in cytochrorine content that appear to be growth $\mathrm{pH}$ dependent. The focus for the coming project year will be on the cloning of genes encoding the $\mathrm{caa}_{3}$-type terminal oxidase and the initiation of studies of expression of those genes. In parallel insertional mutants in this and other terminal oxidases will be isolated from a transposon library which is now available in facultatively alkaliphilic Bacillus firmus OF4. We will also endeavor to isolate menadione-requiring strains from this library that have lost the ability to synthesize menaquinone so that we may attempt to control the level of this respiratory chain component. 


\section{University of Nebraska Lincoln, NE 68588-0118}

124. Physiology and Genetics of Metabolic Flux Regulation in Zymomonas mobilis
T. Conway, School of Biological Sciences $\$ 151,400$ (FY 90 Funds/2 years)

The enzymology of glycolytic pathways is now well established, but the molecular mechanisms that control carbon flux are only beginning to be understood. This research addresses two fundamental biological questions. First, how do the enzymes of glycolytic pathways act in concert to regulate metabolic flux? Second, what is the role of gene expression in regulation of glycolytic enzyme levels and flux? The metabolically simple bacterium, Zymomonas mobilis, has been chosen for this work because it is amenable to genetic studies and the variables involved in regulation of flux can be easily manipulated. This organism uses the Entner-Doudoroff pathway exclusively for conversion of carbohydrates to the sole fermentation products ethanol and carbon dioxide.

Recently, we have found that the $Z$. mobilis genes that encode the glucose facilitated diffusion transporter $(g / \hbar)$, glucose-6-phosphate dehydrogenase ( $z w \hbar), 6$-phosphogluconate dehydratase (edd), and glucokinase $(g / k)$ form an operon. Such an arrangement of genes that govern glucose uptake and the first three steps of the Entner-Doudoroff glycolytic pathway provides the organism with a mechanism for carefully regulating the levels of the enzymes that control carbon flux to 2-keto-3deoxy-phosphogluconate (KDPG), a toxic metabolite. KDPG aldolase (eda) is present at a two-fold higher level than the dehydratase and therefore plays an important role in maintaining low levels of KDPG by consuming this metabolite as a substrate. Expression of the glf-glk operon appears to be coordinated with expression of the unlinked eda gene and the other genes of the glycolytic pathway. Message stability plays an important role in coordinating the levels of the glycolytic enzymes; mRNA half-lives and concentrations are directly proportional to the concentrations of the five most abundant glycolytic enzymes. The relative levels of the rernaining glycolytic enzymes appear to be regulated, at least in part, by the quality of the respective ribosome binding sites and hence the efficiency of translational initiation. Ongoing research involves the relationship of these and other features of glycolytic gene regulation to physiological aspects of glycolytic flux control.

\section{University of Nebraska Lincoln, NE 68588-0118}

\section{Characterization of a Defective Interfering RNA That Contains a Mosaic of a Plant Viral Genome \\ T.J. Morris, School of Biological Sciences and A.O. Jackson, University of California, Berkeley $\$ 182,745$ (2 years)}

This research addresses the identification of viral sequences important in events that effect viral pathogenicity through the molecular characterization of a unique class of symptom modulating RNAs called defective interfering RNAs. DI RNAs are linear deletion mutants of viral genomes that interfere with the helper virus and reduce disease severity. We first discovered DIs in association with tomato bushy stunt virus (TBSV). The molecular characterization of the virus and associated DI RNAs has been completed with the cloning and sequencing of the RNA genomes and the construction of CDNA clones from which infectious RNA transcripts have been produced. This has permitted precise identification of RNA sequences important in replication and encapsidation of Dls by site directed mutagenesis. We have also demonstrated that DI RNAs arise de novo from the viral genome and evolve on continued passage in host plants. We have developed a procedure to permit rapid cloning, sequencing and the evaluation of the biological activity of DI RNAs using PCR amplification methods which will permit a thorough evaluation of the meshanisms of de novo generation and evolution of $\mathrm{DI}$ 
RNAs from cloned inocula. We have also investigated the mechanisms responsible for DI-mediated symptom attenuation by measuring competition between selected DIs isolated from different viruses and by monitoring DI-virus interactions in both protoplasts and whole plants. We have also constructed plasmids that have resulted in the expression of DIs in transgenic tomato plants and are in the process of evaluating the transgenics for tomato decline disease resistance.

\section{University of Nebraska Lincoln, NE 68588}

126. Mechanistic Enzymology of CO Dehydrogenase from Clostridium thermoaceticum
S.W. Ragsdale, Department of Biochemistry $\$ 182,000$ (2 years)

Acetogenic bacteria perform a novel mechanism of $\mathrm{CO}_{2}$ and $\mathrm{CO}$ fixation called the acetyl-CoA pathway. Carbon monoxide dehydrogenase (CODH) assembles acetyl-CoA via methyl-CODH, CODH$\mathrm{CO}$, and acetyl-CODH intermediates. CoA binds to and then is condensed with acetyl-CODH in the final steps of the synthesis. The rates of formation of these intermediates are markedly accelerated by application of a low redox potential $(<-450 \mathrm{mV})$. The origin of the redox dependence is being further investigated. We will further elucidate the reaction mechanisms of the individual steps in the pathway. By steady-state and presteady-state kinetic analyses combined with electrochemical and spectroscopic methods, we plan to further characterize the intermediates. By determining the rates of formation and decay of each of the intermediates and comparing these rates to the overall synthesis and to relevant partial reactions, we plan to establish their kinetic competence. Measulement of the rates of the individual steps should allow us to establish the rate-limiting step(s) in the pathway. The structures and roles of the metal centers in CODH are being investigated with a major focus on the Ni- $\mathrm{Fe}_{3.4}-\mathrm{S}_{4}$ center which appears to play a key role in the assembly of the acetyl moiety of acetyl-CoA.

\section{Ohio State University Columbus, $\mathrm{OH} 43210$}

127. Structure and Regulation of an Archaebacterial Promoter: An In Vivo Study
C.J. Daniels, Department of Microbiology
$\$ 157,575$ (2 years)

The archaebacteria as members of a third evolutionary line of descent, and as inhabitants of some of the most extreme environments known to support life, have the potential to provide novel biochemical systems and insight into the evolution of cellular processes. Full realization of this potential requires the development of in vitro and in vivo systems for the analysis of gene and protein functions. Using a recently described genetic exchange system for the halophilic archaebacteria, we have developed the first plasmid-based expression vector that faithfully directs transcription in vivo. With this system we will establish the sequence requirements for the expression of stable RNA genes in Haloferax volcanii and determine if these genes are regulated by cell growth rate and the nutrient composition of their environment. We will also investigate whather the halophilic transcriptional machinery will recognize the promoter elements of non-halophilic genes. These later studies will indicate the compatibility of archaebacterial transcription signals and show whether the halophiles can act as surrogate hosts for the expression of other archaebacterial genes, such as genes from the extreme thermophiles. 


\title{
Ohio State University Columbus, OH 43210-1292
}

\author{
128. Transmethylation Reactions During Methanogenesis from Acetate in Methanosarcina \\ barkeri \\ J.A. Krzycki, Department of Microbiology \\ $\$ 160,000$ (2 years)
}

Acetate is the primary precursor of methane in many anaerobic environments. One of the initial steps of methane formation from this substrate involves the cleavage of acetyl-CoA by carbon monoxide dehydrogenase; while the terminal step is the reduction of methylated coenzyme $M$ to methane by methylreductase. We are interested in identifying and ordering the proteins and coenzymes involved in the series of transmethylations between these two reactions, using Methanosarcina barkeri as a model organism. Several different components have been implicated as possible methyl level intermediates, including methyltetrahydromethanopterin and one or more corrinoid containing proteins. We have identified four proteins which are methylated by ${ }^{14} \mathrm{C}-2$ acetate in cell extracts when an inhibitor of methylreductase is present. Two of these proteins (of molecular sizes $480 \mathrm{kDa}$ and 29 $\mathrm{kDa})$ have been purified and identified as corrinoid containing proteins; a third (200 kDa) also appears to be a corrinoid protein. Since these methylated proteins accumulate when the terminal enzyme of methanogenesis is inhibited, they may be involved in the transmethylation reactions of acetate dependent methanogenesis. We are testing this by using pulse chase experiments, inhibitor and antibody studies, and determination of the stereochemistry of methyl transfer. We are characterizing the purified proteins, and using them in reconstitution experiments to further test and define their roles in methane formation from acetate or other metabolic pathways of this methylotrophic methanogen.

\section{Ohio State University Columbus, $\mathrm{OH} 43210$}

\section{The Molecular Characteristics of the Lignin-forming Peroxidase L.M. Lagrimini, Department of Horticulture}

The best characterized peroxidase in tobacco is the anionic lignin-forming isoenzyme. A cDNA clone for this peroxidase was isolated from tobacco and joined to the CaMV 35S promoter and transformed into tobacco. This promotes the overexpression of the anionic peroxidase throughout the plant. The cDNA was also inserted in the antisense configuration behind the 355 promoter to suppress the synthesis of peroxidase. Those plants which overproduce peroxidase wilt chronically due to greatly reduced root growth. Transgenic plants which overproduce peroxidase also have a brown discolored cambium, and pith tissue browns rapidly after wounding. The browning can be attributed to the deposition of polyphenolic acids. Unwounded pith tissue from peroxidase overproducer plants was found to have two-fold higher lignin levels. Wound-induced lignification was found to occur in both control and overproducer pith tissue, however, lignification occurred 2 days sooner in overproducer plants. Seed set in plants which underproduce peroxidase was found to be reduced by as much as $98 \%$. The flowers appear normal in morphology with the exception of size. All flower parts (anther, stigma, style) were approximately $17 \%$ larger than in control plants. This size difference was also found in leaf tissue. Pollen was germinated in vitro from control and transformed plants. Control plants showed $>95 \%$ germination while peroxidase underproducer plants had $>65 \%$ germination. Endogenous IAA levels were unaffected in plants which varied in peroxidase activity by 100 fold; however, many of the phenotypes we have observed are thought to be a result of altered auxin levels. 


\title{
Ohio State University Columbus, $\mathrm{OH} \mathbf{4 3 2 1 0}$
}

\author{
130. Structure and Regulation of Methanogen Genes \\ J.N. Reeve, Department of Microbiology
}

$\$ 207,500$ (FY 90 Funds/2 years)

The long-term goals of this project are to characterize the genetic organization and mechanisms of regulation of gene expression in methanogens, to use gene cloning and the techniques of molecular biology to dissect and understand the biosynthesis of methane and to develop genetic exchange systems for methanogens. The regulations of synthesis of methyl coenzyme $M$ reductase (MR) in Methanococcus vannielii and of methyl-viologen reducing hydrogenase (MVH) in Methanobacterium thermoautotrophicum are being studied. Although five, closely-linked genes (mcrBDCGA) form the MR operon only the products of the $m c r B, m c r G$ and mcrA genes are components of the purified MR holoenzyme. We have subcloned and expressed the mcrD and $m c r C$ genes in $E$. coli and now are using antibodies raised against these polypeptides to investigate the locations and functions of the $m c r D$ and $m c r C$ gene products in $M$. vannielii. The $m c r D$ gene product has been purified from $M$. vannielii and its role in methanogenesis in vitro is being determined. The MVH subunits are also encoded by linked genes ( $m v h D G A)$. The product of an additional gene, $m v h \mathrm{~B}$, located immediately downstream of $m v h A$ is predicted to contain $48 \mathrm{Fe}$ atoms in six tandemly-arranged ferredoxin-like domains; a structure we have termed a polyferredoxin. Antibodies raised against the product of a muhB-lacZ fusion have been used to facilitate the purification of the polyferredoxin from $M$. thermoautotrophicum. The role of this molecule as an electron conduit in methanogenesis is being investigated. The cloned $m c r$ and $m v h$ genes are being used as hybridization probes to characterize and quantify transcripts of these genes synthesized in vivo in methanogens growing under a variety of physiological conditions. The effects of environmental and physiological changes on the expression of these genes is being determined. Development of antibiotic resistance conferring determinants and a transformation system for methanogens is an additional major component of this project.

\section{Ohio State University Columbus, $\mathrm{OH}$ 43210-1292}

\section{Regulation of Alternative $\mathrm{CO}_{2}$ Fixation Pathways in Procaryotic and Eucaryotic Photosynthetic Organisms \\ F.R. Tabita, Department of Microbiology

$$
\$ 180,000 \text { (2 years) }
$$

This project addresses the regulation of alternative pathways of photosynthetic carbon dioxide fixation in bacteria and eucaryotic marine algae. Specificilly, we will attempt to determine how the anaerobic photosynthetic bacterium Rhodospirillum rubrum and the aerobic marine diatom Cylindrotheca sp. strain $\mathrm{N} 1$ control the utilization of alternative pathways of $\mathrm{CO}_{2}$ metabolism. Both organisms are representative of important and environmentally significant $\mathrm{CO}_{2}$ utilizing organisms and both organisms have been shown to assimilate $\mathrm{CO}_{2}$ by routes that differ from the usual Calvin reductive pentose phosphate pathway. The proposed studies will employ molecular biological and biochemically-oriented experiments to elucidate the molecular basis for switches in $\mathrm{CO}_{2}$ metabolism. For the studies with $R$. rubrum, we will take advantage of recent strain constructions in which the Calvin $\mathrm{CO}_{2}$ assimilatory pathway has been eliminated. Thus, the significance of the alternative pathway may be evaluated. In addition, other strains of photosynthetic bacteria will allow us to isolate and clone the structural genes of the alternative $\mathrm{CO}_{2}$ fixation system of $R$. rubrum so that the regulation of this pathway may be probed. For the marine diatom, we have already isolated the structural genes encoding the key $\mathrm{CO}_{2}$ fixation enzyme of one $\left(\mathrm{C}_{3}\right)$ pathway. The structural gene(s) encoding the key enzyme of the 
alternative $\left(\mathrm{C}_{4}\right)$ pathway will be isolated to provide a framework for elucidating the molecular regulation of both key genes. In all cases, the molecularly-oriented studies will be supplemented with the appropriate physiological, biochemical, and enzymological studies so that the overall control of $\mathrm{CO}_{2}$ metabolism may be ascertained in these versatile organisms.

\section{Oklahoma State University StIllwater, OK 74078}

\section{The Structure of Pectins from Cotton Suspension Culture Cell Walls}

The overall goal of the project is to characterize as much of the structure of the pectins of the cell walls of cotton suspension cultures as possible. We are solubilizing the pectins from the walls by selective cleavage by either HF solvolysis, endopolygalacturonase hydrolysis or a combination of the two. An additional endoglucanase or strong alkali treatment can be used to solubilize the residual RGI.

In the homogalacturonan regions, we will be determining the distribution of the interspersed rhamnose residues by specifically cleaving at rhamnose residues and determining the lengths of the homogalacturonan fragments on a Dionex Carbohydrate System. The distribution of methyl esterification within these regions will be determined by conversion of the methyl esterified galacturonic acid residues to galactose and then specific fragmentation of resulting mixed polymer and characterization of the fragments.

The sidechains (attached to rhamnose residues) of RGI will be released by treatment with lithium metal in ethylenediamine to destroy the galacturonic acid residues in the backbone of the polymer. The backbone of RGI is being characterized by selective fragmentation using liquid HF.

The oligosaccharides we generate in the experiments described above will be characterized using liquid secondary ion mass spectrometry (LSIMS) and NMR spectroscopy.

\section{University of Oklahoma Norman, OK 73019}

\section{Effect of Community Structure on Anaerobic Aromatic Degradation} M.J. Mclnerney, Department of Botany and Microbiology

The kinetics of benzoate degradation by an anaerobic syntrophic bacterium, Synthrophus buswellii, in coculture with a hydrogen-using sulfate reducer was studied. The maximum specific rate of benzoate degradation was $0.41 \mu \mathrm{mol}$ per $\mathrm{mg}$ of protein per $\mathrm{h}$. Benzoate consumption by thie coculture reached a threshold of 214 to $6.5 \mu \mathrm{M}$ benzoate with no further degradation observed even after extended incubation periods. The addition of sodium acetate, but not sodium chloride, affected the threshold value with higher threshold values $(6.5 \mu \mathrm{M})$ observed at higlier acetate concentrations (16 $\mathrm{mM}$ ). Thermodynamic calculations indicated that the change in free energy approached zero when the threshold value was reached. The existence of a threshold value is an important consideration for modeling the fate of aromatic compounds in anaerobic environments since models that assume a continued first-order decay may fail to predict accurately the fate of the substrate at low in situ concentrations where a threshold for metabolism exists. 
The syntrophic, benzoate-degrading bacterium, $S$. buswellii, was isolated in pure culture using crotonate as the energy source. Also a novel anaerobic bacterium was isolated that catalyzes the cleavage of the aryl ether bond of phenoxyacetate and 2-chlorophenoxyacetate to the corresponding phenol. The bacterium is a gram negative rod that uses a variety of sugars and crotonate. The isolation of these two bacteria will facilitate the study of anaerobic aromatic metabolism in methanogenic environments.

\section{Oregon Graduate Institute of Science and Technology Beaverton, OR 97006-1999}

134. Biochemical Genetics of Lignin Degradation by Phanerochaete chrysosporium
M.H. Gold, Department of Chemical and Biological Sciences Carryover funding

Lignin, the most abundant renewable aromatic polymer, comprises $20-30 \%$ of woody plant cell walls. White rot fungi are primarily responsible for the initiation of the degradation of lignin in wood. The best-studied lignin-degrading organism, $P$. chrysosporium, secretes two extracellular heme enzymes-lignin peroxidase (LiP) and manganese peroxidase (MnP)--which catalyze the oxidative degradation of lignin. The objective of this project is to understand the degradation of lignin and related aromatic compounds at the biochemical and molecular biological levels.

During the past year we have continued to characterize genomic clones for LiP and MnP. We have found that the promoter regions of two different MnP genes contain putative metal response and heat shock elements and that the expression of MnP is regulated by $\mathrm{Mn}$ and heat shock at the level of gene transcription. To study these regulatory systems we have developed the first DNA transformation system for $P$. chrysosporium. During the past year we have developed a homologous DNA transformation system based upon the complementation of an adenine auxotroph with the corresponding Phanerochaete biosynthetic gene. We have also developed a second DNA transformation system based on the complementation of a $P$. chrysosporium uracil mutant with a uracil biosynthetic gene from Schizophyllum commune. Utilizing these systems we intend to develop gene disruption methods for $P$. chrysosporium which should prove indispensable in evaluating the roles of various enzymes in lignin degradation.

We have also continued our studies on the degradation of lignin and other aromatics by LiP and MnP. Recently, we reported for the first time that MnP is able to catalyze significant depolymerization of both hard wood and soft wood lignin. In addition we reported that both the expression of MnP and the degradation of lignin by the white rot fungus Dichomitus squalens are tightly regulated by $\mathrm{Mn}$. These results suggest that MnP plays an important role in lignin degradation. We are continuing to study the pathways by which these enzymes degrade lignin and various related aromatic pollutants. 


\section{Oregon Graduate Institute of Science and Technology Beaverton, OR 97006-1999}

\section{Expansion of Bioconversion Technology at the Oregon Graduate Center M.H. Gold, Department of Chemical and Biological Sciences \\ Carryover funding}

This expansion is establishing a multidisciplinary program for bioconversion research at OGI.

1. Molecular and celiular biology of peroxisomes in methanol-utilizing yeasts J.M. Cregg

Among eukaryotic organisms, the ability to grow on methanol as sole carbon source is limited to a few yeast and fungai species. Methylotrophic yeasts are often found on rotting wood and are thought to oxidize methanol that is released during the degradation of wood by other microorganisms. Key enzymes in the methanol metabolic pathway are known to be compartmentalized in a subcellular organelle called the peroxisome. This organelle exists in all eukaryotes and is the site of hydrogen peroxide-generating oxidative reactions in cells. We are utilizing the methylotrophic yeasts Hansenula polymorpha and Pichia pastoris as model systems to investigate peroxisome biogenesis and function. We have established that peroxisomes are essential for growth on methanol and have isolated mutants that are completely devoid of the organelle. Future plans call for the detailed genetic, biochemical and cellular biological analysis of our peroxisome-deficient mutants and the isolation and analysis of genes required for peroxisome function.

2. Oxidative enzymes involved in fungal cellulose degradation V. Renganathan

Many cellulolytic fungi produce extra-cellular oxidative enzymes in addition to cellulases, but the role of these enzymes in cellulose degradation is not well understood. Such an understanding is necessary for the development of efficient biotechnical processes for the conversion of cellulose to fuels and chemicals. Cellulose-degrading cultures of the white-rot fungus, Phanerochaete chrysosporium produce two extracellular cellobiose-oxidizing enzymes, cellobiose oxidase (CBO) and cellobiose:quinone reductase (CBQase). In the past year, we have developed new procedures for the purification of $C B O$ and $C B Q$ ase. Detailed characterization of $C B O$ and $C B \cap$ ase is in progress. Experiments designed to elucidate the role of these enzymes in lignin and cellulose degradation are planned.

3. Regulatory pathways in filamentous fungi

M.S. Sachs

We are focusing on the molecular mechanisms by which different regulatory pathways interact to control expression of the Neurospora crassa arg-2 gene. This gene encodes the mitochondrially localized small subunit of arginine-specific carbamoyl phosphate synthetase. We are combining genetic and biochemical approaches to define the intragenic and extragenic elements responsible for regulation of arg-2. This gene is regulated by at least three distinct mechanisms: (1) Arg-specific control negatively regulates expression in response to Arg. There is evidence for a translational component to Arg-specific control mediated through a 24 codon upstream open reading frame (UORF) in the transcript. (2) Cross-pathway control (CPC) positively regulates expression in response to limitation for a variety of amino acids including Arg. Elements important for CPC control are found in the promoter region and within introns in the transcript. (3) Developmental control regulates arg-2 expression in response to as yet unidentified developmental signals. 


\section{Oregon Graduate Institute of Science and Technology Beaverton, OR 97006-1999}

136. Chemistry of Ultrathin Membranes and Films

D.H. Thompson, M. Silverberg, S.B. Rananavare, and D.W. Grainger

Department of Chemical and Biological Sciences

Carryover funding

This award completes the current phase of program development within the OGI membrane chemistry initiative. Continued support of the four recent faculty additions listed above has been an essential element for developing this thematically focused group of researchers. Group activities have been aimed at designing a unique curriculum required for student training in this interdisciplinary field and initiating collaborative research projects relating to the structure and dynamics of supramolecular assemblies. Major research interests of the group include:

1. Synthesis and physical characterization of novel ultrathin materials patterned after robust archaebacterial membranes for use in self-assembling organic and organic/inorganic composite materials applications.

2. Mech:anisms of molecular recognition, interfacial binding, and self-assembly in supramolecular systems with special emphasis on the interactions of biologically active materials at nonbiological surfaces.

3. Use of low-angle X-ray, EPR, constant-field and field-cycling NMR techniques to image the molecular organization and dynamics within supramolecular structures and to determine their phase behavior.

4. Modeling of dynamic processes, such as diffusion on two-dimensional lattices, electron and ion transport in complex fluids, and transitions leading to phase separation and microdomain formation, by computational methods and comparing these results with experimental data.

Results from these activities should impact significantly upon development of membrane-based technologies in photoconversion, heterogeneous catalysis, molecular electronics, biomimetic ceramics processing, and medical pharmaceutics/prophylaxis.

\section{Oregon State University Corvallis, OR 97331}

\section{Catalytic Mechanism of Hydrogenase from Aerobic $\mathbf{N}_{2}$-Fixing Microorganisms D.J. Arp, Laboratory for Nitrogen Fixation Research \\ $\$ 142,000$ (2 years)}

We are investigating the catalytic mechanism of the hydrogenase from the aerobic, $\mathrm{N}_{2}$-fixing microorganism, Azotobacter vinelandii. This enzyme efficiently recycles the $\mathrm{H}_{2}$ evolved by nitrogenase. Several properties of this hydrogenase ( 9.9. , a very low rate of the back reaction, $\mathrm{H}_{2}$ evolution, and a low $\mathrm{Km}$ for $\mathrm{H}_{2}$ ) make it ideal to function in an environment in which all of the available substrate is generated in situ. This enzyme is a $\mathrm{Ni}$ - and Fe-containing dimer with subunits of molecular weight 65,000 and 35,000 . This metal content and subunit composition are typical of a large group of $\mathrm{H}_{2}$ oxidizing hydrogenases. We have focused on the catalytic functions of this enzyme and are combining three approaches to understanding how the enzyme functions. First, we are characterizing the mechanisms of a number of inhibitors and inactivators of this hydrogenase including $\mathrm{C}_{2} \mathrm{H}_{2}, \mathrm{O}_{2}, \mathrm{CO}$, $\mathrm{NO}, \mathrm{Cu}^{++}$and $\mathrm{HCN}$. Characterizations include considerations of the competitive nature of the inhibitor, the time-dependence of the inhibition, and the dependence on the redox state of the enzyme. Second, 
we are using EPR and UV-vis spectroscopy to characterize the various inhibited and redox states of the enzyme with a view towards identifying the redox centers in the enzyme and their roles in catalysis. Third, we are using site-directed mutagenesis to study the roles of a number of amino acid residues (cys and his) that are conserved throughout this class of hydrogenases. This system is particularly well-suited to these investigations because the enzyme is well-characterized at the biochemical level and because the bacterium is amenable to genetic transformation.

\section{Oregon State University Corvallis, OR 97331}

\section{Analysis of Potyviral Processing: A basis for pathogen derived resistance?} W.G. Dougherty, Department of Microbiology

Potyviruses are important viral pathogens infecting most crop species. The single-stranded RNA viral genome is expressed initially as a high molecular weight polyprotein which is co- and posttranslationally processed by two viral encoded proteinases (HC-PRO and Nla). The proteolytic activity associated with the Nla proteinase ( 430 amino acids) of tobacco etch virus (TEV) catalyzes proteolytic cleavage at six locations on the polyprotein derived from the TEV RNA genome. The homologous protein (433 amino acids) of tobacco vein mottling virus (TVMV) cleaves at similar, yet distinct, recognition sites. An extended cleavage site sequence has been demonstrated for both proteinases. We examined these proteinases, in a defined cell-free cleavage system, in an attempt to define the biochemical basis of substrate specificity. Each proteinase was specific for its own cleavage site sequence in cell-free trans processing reactions, and no processing of the heterologous cleavage site was evident. This observation held true when the cleavage site sequences were in a natural polyprotein location or moved into a foreign site. Domains of the proteinase which were important in determining this substrate specificity were identified by generating hybrid proteinase genes containing both TEV and TVMV Nla proteinase coding sequences. Using site-directed mutagenesis and standard recombinant DNA techniques, plasmids were constructed which contained coding sequences for hybrid TEV-TVMV proteinases. These plasmids were expressed and tested in a cell-free environment for their ability to cleave both TEV and TVMV substrates. The data suggest that the carboxyl terminal 150 amino acids of the Nla protein contain the necessary information to specifically recognize a particular cleavage site sequence, and that specificity determinants are contained in at least three interactive domains within this region.

\section{University of Oregon Eugene, OR 97403-1229}

\section{Transposon-induced Nuclear Mutations that Alter Chloroplast Gene Expression A. Barkan, Institute of Molecular Biology

The biogenesis of chloroplasts is genetically complex, involving hundreds of genes distributed between the nucleus and organelle. In multicellular plants, developmental parameters confer an added layer of complexity upon the genetic control of organelle biogenesis: the properties of plastids differ dramatically in different cell types. The long term goal of this project is to use mutant phenotypes as a guide to nuclear genes that determine the timing and localization of chloroplast development.

Studies are being conducted with nuclear mutants in maize that are defective in chloroplast translation or RNA metabolism. These mutants are green, but non-photosynthetic due to the absence of several of the enzymatic complexes involved in photosynthesis. We have identified seven transposon-induced nuclear mutants that we feel are likely to define genes involved in plastid translation or RNA metabolism, based upon preliminary studies of their chloroplast proteins, polysome profiles, mRNAs, 
and rRNAs. We are carrying out more detailed molecular studies to better define the defects. Genetic crosses are being performed to map the mutations, to identify preexisting alleles, and to assess the effect of gene dosage on phenotype. Developmental studies will address the timing of gene action during leaf development, and whether the gene acts specifically on chloroplasts or on non-green plastids as well. Molecular clones of several of the most interesting mutations will be isolated by taking advantage of the transposon tag. These clones will be used to obtain the corresponding normal alleles, which will then be used as tools for more detailed functional studies.

\title{
Pennsylvania State University University Park, PA 16802
}

\author{
140. Rapid Regulatory Control of Plant Cell Expansion and Wall Relaxation \\ D.J. Cosgrove, Department of Biology $\quad \$ 173,000$ (FY 90 Funds/2 years)
}

The size and shape of plants depend on cell expansion, which is initiated and maintained by stress relaxation of the cell wall. High-resolution measurements of cell expansion and wall relaxation indicaie that both expansion and relaxation exhibit complex dynamics suggestive of a feedback-controlled process. This feedback process and its relationship to growth control are being explored. The rate of stem elongation in rapidly-growing plants is often observed to fluctuate in complex patterns. Our results indicate that such growth fluctuation is a type of deterministic chaos, resulting from a rapid, nonlinear feedback system operating to control cell expansion. With the use of the pressure-block technique to apply sinusoidal pressure stimuli to the growing stem, we are currently characterizing this feedback system and its alteration by water stress and by different genetic loci for dwarfism. The results require two additions to the conventional model of plant cell growth, namely there must be a growth-rate sensor which integrates growth over 1 min or less, and also a means to alter wall relaxation rapidly (lag of $2-3 \mathrm{~min}$ ) in response to the signal from the growth-rate sensor. Further work will be directed at identifying the nature of the growth-rate sensor and its signal.

\section{Pennsylvania State University University Park, PA 16802}

\section{Role of $\mathrm{Ca}^{++} / \mathrm{Calmodulin}$ in the Regulation of Microtubules in Higher Plants R. Cyr, Department of Biology}

The cytoskeleton, and in particular its microtubule (Mt) component, participates in several processes that directly affect growth and development in higher plants. Normal cytoskeletal function requires the precise and orderly arrangement of Mts into several cell cycle and developmentally specific arrays. One of these, the cortical array, is notable for its role in somehow directing the deposition of cellulose, the most prominent polymer in the biosphere. Unfortunately, little molecular information is available regarding the formation of these arrays or the cellular signals to which they respond. It is therefore important to acquire information regarding the molecules which regulate the appearance of Mts within the different arrays. Experimental data has been obtained to suggest that plant cells use calcium, in the form of a $\mathrm{Ca}^{*} / \mathrm{calmodulin}$ complex, to affect the polymerization state of Mts in the cortical array. Owing to the importance of $\mathrm{Ca}^{++}$as a regulatory ion in higner piants we are probing for a putative $\mathrm{Ca}^{++} / \mathrm{Mt}$ transduction pathway which may serve to integrate $\mathrm{Mt}$ activities within the growing and developing plant cells. To aid in our investigations we have developed a lysed cell mociel, utilizing carrot protoplasts, that permits functional assays to be performed and we are using it to identify the levels of $\mathrm{Ca}^{++}$and calmodulin affecting the various arrays. We are also in the process of identifying and characterizing the relevant proteins involved in this putative regulatory pathway. The information gained in these studies will be useful in understanding how developmentally important signals are transduced into morphogenic events during plant growth and development. 


\section{Pennsylvania State University University Park, PA 16802}

142. Enzymology and Molecular Biology of Lignin Biodegradation

M. Tien, Department of Molecular and Cell Biology

$\$ 84,000$

The white rot fungus Phanerochaete chrysosporium secretes a large number of enzymes for the purpost of degrading lignin. These include the lignin peroxidases, Mn-dependent peroxidases and enzymes for 'iydrogen peroxide production. Our research efforts are aimed at understanding the mechanism and interaction of thes: enzymes and how they are regulated on the molecular level. The active site of these peroxidases is being characterized by a variety of kinetic and equilibrium techniques. We are particularly interesicd in how the protein affects the electron density of the heme and the mechanism of peroxide activation. These mechanistic studies complement our studies on how these isozymes are regulated. Isozyme patterns change in response to different environmental conditions. Having cloned many of the genes encoding these peroxidases, we are in the process of studying regulation on the molecular level.

\section{University of Pennsylvania Philadelphia, PA 19104}

\section{Circadian Rhythms in CAB Gene Expression}

A.R. Cashmore, Plant Science Institute, Department of Biology

Many phenomena in nature exhibit cyclic oscillations in activity with a period of approximately 24 hours. These circadian rhythms are controlled by what is commonly referred to as the circadian ci')ck. An example of such a rhythm is the daily variation in the level of mRNA encoding the chlorophyll $a / b$ binding $(C A B)$ polypeptides. We and others have recently demonstrated that this circadian oscillation in $C A B$ gene expression reflects rhythmic changes at the level of transcription - the genes are transcribed during the day but not at night. This project is concerned with defining the nature of the promoter sequences and associated factors that mediate this transcriptional response.

Our initial studies will be concerned with defining the nature of the promoter sequences that mediate circadian oscillations in expression. Of particular interest will be the determination of whether these circadian response elements (CREs) are distinct from those that mediate light-induced expression associated with de-etiolation. These studies will concern both the cab-E gene from Nicotiana plumbaginifolia and the $\mathrm{CAB}$ genes from Arabidopsis thaliana.

We have recently reported a study of DNA binding proteins for the Nicotiana plumbaginifolia cab- $E$ promoter. Although rather comprehensive, this study as by no means exhaustive. We will extend these studies to include a more detailed analysis of ine factors that bind to the CRE region of this promoter. We will attempt to characterize a factor(s) that bind to the CRE. If we can cha acterize such a factor than we will determine whether the activity of this factor can be correlated with rhythms in expression. These studies will be aimed at the eventual cloning of the gene sncoding this CREelated factor. 


\title{
University of Pennsylvania Philadelphia, PA 19104-6018
}

\author{
144. Membrane-Attached Electron Carriers in Photosynthesis and Respiration \\ F. Daldal, Department of Biology \\ $\$ 205,635$ (2 years)
}

The overall aim of this project is to contribute to a clear and complete understanding of various energy transduction pathways that operate during respiration and photosynthesis. Our model system is that of the facultative photosynthetic bacterium Rhodobacter capsulatus which provides an excellent experimental system for multidisciplinary approaches. In this organism, electron transfer during photosynthetic growth had been thought to proceed only via a cyclic pathway through the membranebound cytochrome (cyt) $b c_{1}$ complex to the photochemical reaction center, mediated by the soluble cyt $c_{2}$. Genetic and biochemical experiments involving the deletion of the gene encoding this protein have firmly established that both respiratory and photosynthetic growth continues in the absence of the soluble cyt $c_{2}$. Electrons are still transferred from the cyt $b c_{1}$ complex to the reaction center, but the molecular nature of the carrier responsible of it is unknown. Our hypothesis is that in the absence of cyt $c_{2}$ electrons are transferred from the cyt $b c_{1}$ complex to the reaction center via a membraneattached carrier. A genetic approach based on the complementation of mutants lacking cyt $c_{2}$ has revealed the existence of membrane-attached electron carriers structurally distinct from, and yet functionaliy similar to, cyt $c_{2}$. Our specific goal is to identify and clone the component(s) involved in this "soluble electron carrier" (i.e., cyt $c_{2}$ )-independent electron transfer pathway, and characterize their structure and function in respiration and photosynthesis using both genetic and biochemical approaches.

\section{University of Pennsylvania Philadelphia, PA 19104-6018}

\section{Transport Function and Reaction Mechanism of Vacuolar $\mathbf{H}^{+}$-Translocating Inorganic Pyrophosphatase \\ P.A. Rea, Department of Biology \\ $\$ 171,998$ (2 years)}

The vacuolar membrane of plant cells contains two primary $\mathrm{H}^{+}$pumps: a $\mathrm{H}^{+}$-ATPase and a $\mathrm{H}^{+}$translocating inorganic pyrophosphatase $\left(\mathrm{H}^{+}\right.$-PPase). Both enzymes catalyze inward, electrogenic $\mathrm{H}^{+}$translocation (from cytosol to vacuole lumen) to establish a $\mathrm{H}^{+}$-electrochemical gradient across the vacuolar membrane capable of acting as energy source for the secondary, $\mathrm{H}^{+}$-coupled transport of a wide range of solutes. The basic question of why there should be two transport systems (the ATPase and PPase) pumping the same ion $\left(\mathrm{H}^{+}\right)$into the same intracellular compartment therefore arises.

Our research program is directed at answering this question by mechanistic analyses of native membrane vesicles isolated from Vigna radiata - one of the richest known sources of vacuolar $\mathrm{H}^{*}$ PPase - and by reconstitution of the isolated enzyme into artificial membranes of defined ionic conductance. Towards this end we have isolated the enzyme, inserted it into liposomes and reconstituted its capacity for $\mathrm{K}^{+}$-dependent $\mathrm{H}^{+}$-translocation. Reconstituted preparations of the functionally integral pump should enable: (1) Estimation of its $\mathrm{H}^{+}$-substrate (PPi) stoichiometry and capacity for reversal. (2) Elucidation of the enzyme's capacity for $K^{*}-t$ translocation. (3) Analysis of the response of the $\mathrm{H}^{+}$-PPase to potential cytosolic modulators without interference from independent conductance pathways. (4) Investigations of the vectorial operaticn, organization and modulation of the pump in an innerentily sided prepalation. (5) Dissection of the partial reactions constituting the catalytic cycle and enumeration of their effector and ligand requirements. In conjunction with the 
sequence and topographic data deriving from our recent molecular cloning of the gene encoding the catalytic subunit of the $\mathrm{H}^{+}$-PPase, these objectives will provide insight into the role played by the $\mathrm{H}^{+}$PPase in cellular PPi stasis and energized $\mathrm{K}^{+}$transport and an understanding of the mechanisms underlying these processes.

$\mathrm{H}^{+}$-translocating PPase have been identified in the energy-coupling membranes of mitochondria, chloroplasts and bacteria but the tonoplast $\mathrm{H}^{+}$-PPase is the first example of a PPi-dependent $\mathrm{H}^{+}$translocase in a "non-energy" coupling membrane. In view of the apparent ubiquity of the $\mathrm{H}^{+}$-PPase in the vacuolar membranes of plant cells, the unique status of PPi as the limiting case of a high energy phosphate and the increasing recognition of PPi as a key metabolite in plant cells, it is expected that many of the conclusions deriving from this work will be of broad bioenergetic applicability.

\section{Purdue University West Lafayette, IN 47907}

\section{Structure and Biosynthesis of the Mixed-linkage B-D-Glucan of Grasses \\ N.C. Carpita, Department of Botany and Plant Pathology \\ $\$ 166,000$ (2 years)}

We investigate the chemical structure and biosynthesis in vitro of the mixed-linkage B-D-glucans, cell wall polymers unique to cereal grasses. The general structure is a worm-like polymer of a cellotriosyl and cellotetraosyl units connected by single $(1 \rightarrow 3)$ B-D-linkages. Regions of contiguous $(1 \rightarrow 3)$ linkages and longer stretches of $(1 \rightarrow 4)$ linkages susceptible to cleavage by special endoglucanohydrolases are also built into the polymer at special points. Determination of the fine structure of B-D glucans is made by HPLC separation of a large number of glucosyl oligomers released as digestion products of the B-D-glucans by purified endo-glucanases. Identification of the oligomers will then be made by secondary enzymic hydrolysis and methylation analysis by g.l.c.-m.s. These techniques will permit more detailed investigations of the diversity of B-D-glucan structure among cereal grasses and selected tissues. Our improved procedures for isolation of the Golgi apparatus coupled with the analytical advancements will also permit investigation of the factors involved in the synthesis of this complex polymer in vitro. Hydrolysis of the radioactive reaction products by B-Dglucan-selective endoglucanases and separation of the diagnostic oligomers by HFLC has already provided us unequivocal evidence for synthesis of this polymer in vitro. The enzyme(s) remain active for several hours, are optimal in $\mathrm{mM}$ UDPG, are stimulated by $\mathrm{Mg}^{2+}$ selectively and inhibited by $\mathrm{Ca}^{2+}$. Preliminary data show that the divalent effectors also influence the ratio of $3 \mathrm{mer}$ and $4 \mathrm{mer}$ unit structures found in B-D-glucan.

\section{Purdue University West Latayette, IN 47907}

\section{7. $\mathrm{Ca}^{++}$Gated Proton Fluxes in Energy Transducing Membranes} R.A. Dilley, Department of Biological Sciences

Our research on chloroplast bioenergetics focuses on the proton electrochemical potential gradient that forms as an intermediary energy storage in, or across, the thylakoid membrane, during the conversion of absorbed sunlight energy into the chemical energy forms needed for plant life. The proton gradient - akin to a battery but powered by protons $\left(\mathrm{H}^{+}\right)$rather than electrons - drives the energy-requiring synthesis of adenosine triphosphate (ATP) as the protons flow down the energy gradient, through a special membrane protein complex that "coup!es" the energy-releasing $\mathrm{H}^{+}$flow to the energy-requiring ATP formation reaction. The molecular mechanisms for $\mathrm{H}^{+}$ion movement in and through membranes are not understood, and represent an important, unsolved question in biology. 
We have evidence that the proton flow though the energy coupling complex is a "gated" flux, with calcium ions providing part of the mechanism for switching the $\mathrm{H}^{+}$flux gate between the open or closed condition. It appears that when calcium ions are tightly bound to the $8 \mathrm{kDa}$ subunit of the $\mathrm{H}^{+}$ channel part of the coupling complex, the $\mathrm{H}^{+}$stays within localized domains, but when the calcium ions are displaced, the $\mathrm{H}^{+}$ions flow out of the postulated gate into the inner volume of the thylakoid and form a proton gradient over a larger volume, thus the term delocalized proton gradient is used. We are studying the biochemical parameters that control the calcium gating action on the $\mathrm{H}^{+}$gradients.

\section{Purdue University West Lafayette, IN 47907}

\section{Regulation of Photosynthetic Membrane Components in Cyanobacteria L.A. Sherman, Department of Biological Sciences}

The major objectives of this proposal are to analyze gene regulation under different environmental conditions and to determine the role of the psbO protein (MSP, the manganese stabilizing protein, the $33 \mathrm{kDa}$ protein) in $\mathrm{O}_{2}$-evolution. These objectives are studied in the transformable cyanobacteria Synechococcus sp. PCC7942 and Synechocystis sp. PCC6803, respectively. We have produced a deletion strain $(\triangle p s b O)$ in Synechocystis that completely lacks the gene or the gene product, and which can grow photosynthetically at about $2 / 3$ the normal rate. We have used this deletion strain to construct site-directed mutations at specific, externally-located, charged residues. In particular, we will analyze rissidues and domains that enable MSP to interact with the PSII reaction center components.

We have identified and cloned the gene which codes for the novel chlorophyll- protein complex which is synthesized during iron deficiency. The apoprotein, isiA, has an amino acid sequence very similar to that of the gene product of $p s b C$ (CP43) except for the lack of most of the large lumenal loop $E$. We have found that an insertion mutant in isiA can grow normally in regular iron-sufficient medium and in partially iron-deficient cultures. We have also identified a putative candidate ior irpR, the ironregulated DNA binding protein that is used to control gene expression based on iron concentrations. During the coming year, we will continue to analyze gene regulation in iron-deficient conditions, as well as the role of isiA in PSII function and in membrane assembly.

\section{Rockefeller University New York, NY 10021-6399}

149. Asparagine Synthetase Gene Family: Differential Expression During Plant Development G. Coruzzi, Laboratory of Plant Molecular Biology

$\$ 94,000$

Asparagine synthetase ( $A S$ ) plays a key role in plant development, as asparagine is one of the two major nitrogen transport compounds in plants. While $A S$ has been the subject of biochemical investigations for several decades, problems with enzymatic instability in vitio have left basic questions unanswered concerning the regulation of asparagine biosynthesis. We have utilized a molecular approach to isolate $A S$ genes from the legume Pisum sativum and have used the clones as probes to analyze the factors which regulate asparagine biosynthesis. Our studies have shown that there are at least two plant $A S$ genes ( $A S 1$ and $A S 2$ ) which each encode glutamine-dependent cytosolic $A S$ enzymes. While there are some differences in the expression patterns of $A S 1$ and AS2, the AS1 gene is most highly expressed in aii organs examined. Most surprising is the finding that light rapidly suppresses the transcription of $A S 1$ through a phytochrome-mediated response. This expression pattern coincides with the fact that asparagine, which has a higher $\hat{N}: \bar{C}$ ratio than giularnine, is the preferred nitrogen transport compound synthesized in conditions where carbon skeletons are limiting (e.g. in the dark). Light also has a dramatic effect on AS1 gene expression in non-photosynthetic 
organs such as roots. By shielding roots from direct light, we have shown that the small amount of light which penetrates the soil is able to turn off transcription of the AS1 gene in roots. AS1 expression is induced to high levels irrespective of light in organs where asparagine serves to mobilize large amounts of nitrogen (e.g. cotyledons and nitrogen-fixing root nodules). We are currently characterizing the nucleotide sequence and transcription start sites of the AS1 and AS2 promoters. The AS promoters will be fused to the GUS reporter gene and introduced into transgenic tobacco. Analysis of the function of full length AS promoters will allow us to determine the cell types in which the $A S$ genes are expressed. Analysis of promoter deletion mutants will allow us to define the cisacting DNA elements involved in negative regulation by light.

\section{Rutgers University \\ New Brunswick, NJ 08903-0231}

150. Cellulase - A Key Enzyme for Fermentation Feedstocks

D.E. Eveleigh, Department of Blochemistry and Microbiology

$\$ 146,000$ (FY 90 Funds/2 years)

Feedstock chemicals can be obtained from biomasss, cellulose forming the prime substrate with regard to its abundance. Cellulose would be best utilized through fermentation, but must first be converted to sugars. For this purpose we have been studying the cellulase of a thermophilic bacterium, Microbispora bispora. This actinomycete produces a cellulase that is effective towards crystalline cellulose, is thermally stable and is comprised of endo- and exo-splitting glucanases and also beta-glucosidase. The latter is resistant to end-product inhibition which makes it potentially attractive for industrial application. We find two beta-glucosidases - two distinct genes and gene products. The latter differ in their substrate specificities. These genes are being sequenced in order to clarify understanding of mechanism of action of the enzyme. M. bispora additionally produces an exo-splitting glucanase, to date a relatively rare bacterial enzyme. It is important as it is synergistic with endo-glucanases in its action towards crystalline cellulose. Through cloning one cellobiohydrolase has been gained, selected through the use of a sensitive monoclonal antibody assay. The basic characterization of the cellobiohydrolase produced in E. coli has been accomplished. Yields were low, but through cloning into Streptomyces lividans as a host, an increase in yield of 10-15 fold was gained. The Streptomycete cloned enzyme is slightly larger than that from $E$. coli probably due to glycosylation, and it is also more thermally stable. Its gene sequence has been determined. Such sequences allow evolutionary analysis of cellulase genes - 60 sequences are now known. Our analysis of them indicates that the domains (binding and catalytic) could have evolved independently, and later merged. The application of these unique cellobiohydrolases and beta-glucosidases is being improved and should considerably aid in total cellulolysis.

\section{Rutgers University}

New Brunswick, NJ 08903

151. The Role of Alternative Respiration in Plants

I. Raskin, AgBiotech Center

$\$ 74,000$

Plants are generally considered poikiloterms (organisms whose temperature is determined by the environment). Thermogenic plants, which can generate large amounts of heat in their inflorescences via the cyanide-insensitive or alternative respiratory pathway (ARP), are the only accepted deviation from this rule. In partial challenge to this view, we propose that, while most plants do not effectively thermoregu!ate at the organismic leve!, they still do so at the mitochondrial level. This implies that the difference in heat production and thermoregulation between so-called "thermogenic" and "nonthermogenic" plants is quantitative rather than qualitative. We suggest that the increased "heating" 
of the inner mitochondrial membrane at low temperatures is accomplished by a larger electron flux through the ARP, which dissipates most of the energy stored in the respiratory substrates as heat. This localized thermoiegulation counteracts the deleterious effects of chilling on the fluidity of the mitochondrial membrane, which determines the activity of the respiratory enzymes vital for plants. Published observations provide support for our hypothesis by demonstrating an inverse correlation between the ARP activity and temperature. We will use a state-of-the-art heat-conductance microcalorimeter, adapted for use with biological samples, to measure ARP activity and heat evolution.

\section{Rutgers University \\ Piscataway, NJ 08855-0759}

\section{Corn Storage Protein: A Molecular Genetic Model J. Messing, Waksman Institute}

$\$ 204,572$ (FY 90 Funds/2 years)

Corn is the staple crop for animal feed worldwide. The corn kernel is rich in oils, carbohydrates, and proteins. Most of these macromolecules are digested in the gut and are the essential source of fatty acids, sugars, and amino acids. The latter ones are more complex because nonruminants are unable to interconvert certain amino acids. Therefore, the amino acid composition of the corn kernel directly determines the nutritional value of the feed. Since ariirio acid composition is not balanced in the seed of one plant, a diet of corn and soybean meal can be used. Still, one amino acid, namely methionine, remains too low. Therefore, a diet of animal feed is supplemented with free methionine. This supplement, however, has to be derived by fermentation, an energy costly process. Consequently, a higher level of methionine in the seed would create an important energy saving step. Recently, we have isolated a storage protein gene from corn that contains about $23 \%$ methionine and is called the $10 \mathrm{kD}$ zein gene. It is a single copy gene in contrast to the multigenic zein family that we have studied previously. We have now found two trans-acting factors that are absent in most inbreds. Both aid in the overexpression of the $10 \mathrm{kD}$ protein and lead to increased methionine levels in the mature seed of BSSS-53. They both act at the post-transcriptional level. Only one of them, called Zpr10/(22), which appears to act at a later step in $10 \mathrm{kD}$ zein expression than the other one, has been mapped so far. A very tightly linked DNA probe has now been constructed and is used to further refine the map position of Zpr10/(22) so that its gene can be cloned by linkage. Cloning of this regulatory gene should provide interesting insight into post transcriptional regulation of gene expression and be useful to control the accumulation of methionine in maize kernels.

\section{Salk Institute for Biological Studies San Diego, CA 92138-9216}

\section{Genetic Analysis of Photoreceptor Action Pathways in Arabidopsis thaliana J. Chory, Plant Biology Laboratory}

The process of greening, or differentiation of the chloroplast, involves the coordinate regulation of many nuclear and chloroplast genes. The cues for the initiation of this developmental program are both extrinsic (e.g., light) and intrinsic (cell-type and plastid signals). Several regulatory photoreceptors are involved in the perception of light signals; however, the exact mechanisms by which light and other signals are perceived by plant cells and converted into molecular genetic information are not understood. We have identified Arabidopsis thaliana mutants in both signal perception and transduction elements of these pathways based on aberrant morphological phenotypes in response to light. We are using a combination of genetics, molecular biology, and biochemistry to elucidate the precise biochemical lesion in these mutants and to begin to dissect the number and interactions of signal transduction pathways involved in light regulation of plant development. 
In order to identify mutants that are not dependent on a pre-determined phenotype, we are using promoter fusions to select trans-acting regulatory mutations. We have introduced an Arabidopsis lightactivated cab promoter fused to both screenable and selectable markers into Arabidopsis using Agrobacterium tumefaciens transformation. The transgenic line has been mutagenized to allow identification of mutations where the cab promoter is aberrantly expressed. We are currently analyzing mutants in which the cab promoter is expressed inappropriately with regard to tissue-specific expression, light-dark expression, or in response to chloroplast signals. Detailed biochemical and genetical characterization of these potentially interesting regulatory mutants is in progress.

\section{Scripps Clinic and Research Foundation La Jolla, CA 92037}

\section{Genetic Engineering with a Gene Encoding a Soybean Storage Protein R.N. Beachy, Department of Cell Biology \\ $\$ 210,000$ (2 years)}

Genes that encode the soybean seed storage proteins are tightly regulated and are expressed only during seed maturation. We have characterized the DNA sequences that control expression of the genes encoding the $\alpha^{\prime}$ and B-subunits of B-conglycinin. The promoters, and promoter mutants (created by in vitro mutagenesis) have been tested both in transgenic tobacco plants and by in vitro assays. The in vitro assays documented that nuclei isolated from developing seeds, but not from somatic tissues, contain proteins that bind to the promoters. After localizing the DNA sequences to which proteins bind, a large number of mutations in the cis-acting DNA sequences were made: the mutant promoters were ligated to a reporter gene and introduced into transgenic plants. In our analysis of transgenic plants we found that some elements that bound proteins were "silencers" of expression rather than enhancers. Other sequences that apparently bound no proteins are apparently essential to promote gene expression. These results demonstrate the importance of carrying out in vivo as well as in vitro experiments during the analyses of transcriptional promoters. During the ongoing research period we will identify those DNA sequence elements that both activate as well as silence the expression of the $\alpha^{\prime}$ and B-subunit promoters, and the role that abscisic acid and sulfur amino acids play in their regulation.

In a second aspect of this work, we are studying the use of these transcriptional promoters to decrease the seed specific expression of several target genes. This is being done by causing the accumulation of antisense RNAs of the targeter gene.

\section{Solar Energy Research Institute Golden, CO 80401}

\section{The Water-Splitting Apparatus of Photosynthesis}

M. Seibert, Photoconversion Research Branch

The $\mathrm{O}_{2}$-evolving system of photosystem II (PSII) binds a tetrameric manganese cluster required for catalyzing water-splitting function. Bridging ligands hold the cluster together, but terminal ligands bind it to at least some of the proteins comprising the PSII D1-D2 reaction center (RC) complex. Conditions have been found under which either one or four $\mathrm{Mn}$ per reaction center, detected by direct metal analysis can bind with high affinity to PSII membranes depleted of functional manganese. Under the former conditions, $\mathrm{Mn}^{2+}$ acts as a donor to PSII, but under the latter, four independent high affinity, $\mathrm{Mn}$ binding sites are detected by an assay involving the noncompetitive inhibition by $\mathrm{Mn}^{2+}$ of light-induced diphenylcarbazide (DPC) donation to PSII. The DPC concentration seems to determine ie type of interaction between $\mathrm{Mn}^{2+}$ and PS!!. The four above-mentioned high affinity. Mn-binding sites are located on the lumenal membrane side of PSII RC proteins and are protected specifically by $\mathrm{Mn}^{2+}$ from 
the effects of amino acid modifiers. Carboxyl or histidyl amino acid residues are each associated with two of the four high affinity sites. Only the carboxyl sites are observable when the two more loosely bound of the four functional manganese are removed from the membrane. Protease and amino acid modifier studies suggest His-337 on the D1 protein as a primary candidate for one of the histidine sites. The four high affinity sites may be sites associated wiih photoactivation and might also provide some terminal ligands for functional manganese.

\section{Southern Illinois University Carbondale, IL 62901}

\section{Regulation of Alcohol Fermentation by Escherichia coli D.P. Clark, Department of Microbiology}

$$
\$ 172,000 \text { (2 years) }
$$

The purpose of this project is to elucidate the way in which the synthesis of ethanol and related fermentation products are regulated in the facultative anaerobe Escherichia coli. We are also investigating the roles of certain genes which are induced during fermentative growth. Structural and regulatory mutations affecting the expression of the fermentative alcohol dehydrogenase have been isolated. The adh structural gene has been cloned and sequenced; at present the upstream sequences responsible for anaerobic induction are being characterized. The protein encoded by the adh gene expresses both alcohol and acetaldehyde dehydrogenase activities. The latter of the two activities may be specifically abolished by deleting the $\mathrm{C}$-terminal region of the protein. Further delineation of the structure-function relationships of the $A D H$ protein is being performed by the polymerase chain reaction (PCR) approach. The PCR method is also being used to generate hybrids between the adh genes of $E$. coli and related gram negative bacteria. The kinetics of hybrid enzymes will be assessed and correlated with sequence alterations. The ldh gene, encoding the fermentative lactate dehydrogenase has also been cloned and sequencing is in progress. Regulatory mutations which affect the expression of LDH are also available. Many anaerobically induced gene fusions can be switched on in air in the presence of cyanide or chelating agents. The possibility of an iron containing regulatory protein is being investigated.

\section{Stanford University Stanford, CA $94305-5020$}

\section{Nodulation Genes and Factors in the Rhizobium Legume Symbiosis} S.R. Long, Department of Biological Sciences

$\$ 310,000$ (2 years)

We are studying the symbiosis between Rhizobium bacteria and plants. This interaction results in the formation of nitrogen fixing nodules on plants such as soybean, alfalfa, and other plants. The detailed understanding of how these symbioses work is critical for possible extended uses of Biological Nitrogen Fixation for energy conservation. Our research is concerned with determining how the nitrogen fixing symbiotic bacteria accomplish the early steps of inducing nodule clevelopment on the plant host. We aim to characterize signals produced by Rhizobium meliloti that are active in causing the early host responses on roots of alfalfa (Medicago sativa). We have isolated several Nod-factors whose molecular weight and mass-spectrophotometric fragments suggest they are tetramers alid pentamers of $\mathrm{N}$-acetyl-glucosamine, sometimes modified with an acetyl group, an acyl group and/or with sulfate. We have shown that two of the nodulation genes, nodP and nodQ, carry out the activation of sulfate by combination with APS, consistent with an enzymatic role in synthesis of the 
Nod-factors. This is the first in vitro demonstration of any nodulation gene function. We are currently pursuing the function of several other genes. We have also shown that Rhizobium cells, and also chemical Nod-factors, are able to provoke relatively rapid (1-2 minutes) depolarization of the membrane potential in root hair cells. This response is not seen with Nod bacteria, nor with a nonhost plant. Based on this initial research result, we will pursue further the nature of plant responses to the nodulation signals from Rhizobium.

\section{Stanford University Stanford, CA 94305}

\section{Enzymology and Molecular Biology of Cell Wall Biosynthesis P.M. Ray, Department of Biological Sciences}

$\$ 195,000$ (2 years)

Based on the recent findings of a few promising polypeptide candidates for Golgi-localized glucan synthase-I (UDPG:B-1,4-glucan glucosyl transferase) and plasma membrane associated glucan synthase-II (UDPG:B-1,3-glucan glucosyl transferase), it is proposed to extend the fractionation and immunological approaches to verify the selected candidates, identify other GS-I candidate(s) using purified Golgi, and clone the gene sequences for each of these candidates. The cloned gene sequences will be characterized by anti-sense mRNA inhibition oi the synthesis of respective cell wall components by transient gene expression assays using pea tissue protoplasts. Identified clones will later be used to develop recombinant tomato plants carrying antisense gene(s) under the control of an inducible promoter to determine the function of such clones.

\section{Texas A\&M University College Station, TX 77843-2128}

\section{Nuclear Genes Encoding Plastid Proteins Expressed Early in Chloroplast Development J.E. Mullet, Department of Biochemistry and Biophysics \\ $\$ 76,000$}

The long term objective of this research is to elucidate mechanisms which regulate plastid number and composition in higher plants. This proposal will focus on the identification and characterization of nuclear genes encoding plastid proteins which are expressed early in chloroplast development. In barley, early events in chloroplast biogenesis such as plastid replication, DNA synthesis and activation of plastid transcription occur in basal cells of the developing leaf. The initiation of these processes precedes accumulation of the photosynthetic apparatus which occurs as cells mature and are displaced apically in the leaf. Spatial separation of successive phases of chloroplast biogenesis in barley leaves has been used to identify chloroplast DNA-binding proteins which are expressed early in chloroplast biogenesis. Furthermore, it has been found that inhibition of the activation of plastid transcription during early chloroplast biogenesis inhibits expression oí nuclear genes such as cab and rbcS but not the accumulation of plastid DNA. Genes which encode plastid nucleic acid binding proteins or enzymes involved in DNA synthesis are being sought. Once identified, the cis- and transfactors which regulate these genes will be elucidated. 


\section{Texas Tech University Lubbock, TX 79409}

160. Characterization of a 1,4-B-D-glucan Synthase from Dictyostelium discoideum
R.L. Blanton, Department of Biological Sciences $\$ 145,765$ (FY 90 Funds/2 years)

The biochemical and molecular biological analysis of eukaryotic cellulose synthesis has been hindered by the absence of a reliable, fully characterized in vitro assay for the cellulose synthase. The objective of this project is to characterize further the 1,4-B-D-glucan synthase we have obtained from the cellular slime mold Dictyostelium discoideum. This organism possesses many advantages as a model organism for studying cellulose synthesis: there is an in vitro assay system for the cellulose synthase, cellulose synthesis is induced, mutants may be readily selected, cultural manipulations are diverse and simple, and there exists a significant body of techniques and protocols for the experimental manipulation of this organism. Our expectation is that at least some of what we learn will be applicable to understanding higher plant glucan synthases.

In this first year of the project we have continued or initiated several projects, all of which are still in progress. These projects include: (1) the characterization of potential antibody and nucleic acid probes for the cellulose synthase; (2) the study of the developmental regulation of the cellulose synthase; (3) the development of a solubilization procedure for the enzyme activity; (4) the development of screening procedures for detecting cellulose-deficient mutants; (5) the development of more rapid and sensitive enzyme assay procedures; (6) the study of the interactions between cellulose and the extracellular matrix proteins of the Dictyostelium stalk; and (7) the continued characterization of the enzyme and the in vitro product.

\section{Texas Tech University Lubbock, TX 79409}

\section{The Interaction of Ferredoxin:NADP ${ }^{+}$Oxidoreductase and Ferredoxin:Thioredoxin Reductase with Substrates \\ D.B. Knaff, Department of Chemistry and Biochemistry $\$ 158,250$ (FY 90 Funds/2 years)}

Eleven monoclonal antibodies against spiılach ferredoxin: $\mathrm{NADP}^{+}$oxidoreductase have been prepared and shown to inhibit electron flow from NADPH to the non-physiological acceptor, 2-6-dichlorophenol indophenol (DCPIP) without inhibiting electron flow from NADPH to ferredoxin. Spectral perturbation measurements confirmed that none of the antibodies interfered with the binding of either ferredoxin or NADP ${ }^{+}$to the enzyme. These results have been interpreted in terms of the presence of a highly antigenic binding site for DCPIP on FNR, distinct from the binding sites for either of its physiological substrates. The interaction of FNR with ferredoxin is under investigation, using differential chemical modification to identify specific amino acids involved in electrostatic interactions between the two proteins. Complex formation between FNR and ferredoxin protects carboxyl groups on ferredoxin against modification by taurine in the presence of a water-soluble carbondiimide and protects lysine -amino groups on FNR against biotinylation. The ferredoxin acidic amino acids and FNR lysines protected, presumably because of their involvement in complex formation, will be identified by peptide mapping. Antibody and cross-linking techniques have been utilized to demonstrate that the ferredoxinbinding site on the ferredoxin-dependent glutamate synthase of spinach chloroplasts is similar to that found on FNR. Resonance Raman spectroscopy, magnetic circular dichroism and electron paramagnetic resonance spectroscopy have been used to demonstrate that spinach glutamate $\mathrm{S}^{\prime}$, nthase contains a [3Fe-4S] cluster and that spinach ferredoxin:thioredoxin reductase contains a [4Fe-4S] cluster. 


\section{Virginia Polytechnic Institute and State University Blacksburg, VA 24061}

\section{Enzymology of Acetone-Butanol-Isopropanol Formation}

Acetone, 1-butanol, and isopropanol (solvents) are important industrial chemicals and fuel additives. Several Clostridium species produce butanol as a major product. $C$. beijerinckii (also known as $C$. butylicum) which is used in this study, can produce all three compounds. Solvent production by clostridia requires a metabolic switch (from acid production to solvent production) that involves the induction of solvent-forming enzymes. The expression of solvent-production genes depends on certain environmental stimuli, and the ratio of solvents produced is also affected by environmental conditions. The objective of this project is to elucidate the mechanisms that control the expression of solventproduction genes and that control the product ratio. Our approach is to elucidate first the properties of solvent-forming enzymes and then to use the biochemical data to support the use of genetic tools to study the control mechanisms. We have studied enzymes for all solvent-forming reactions and also several enzymes catalyzing related reactions. Our study of enzymes has emphases on (1) alcohol dehydrogenases (ADHs) that catalyze the formation of butanol, isopropanol, and ethanol, and (2) enzymes reacting with acetoacetyl-CoA and leading to either butanol or isopropanol formation. We have identified three distinct ADHs: a primary/secondary ADH from strains that produce both butanol and isopropanol, and two different primary ADHs from a strain not producing isopropanol. The enzymic properties of the three ADHs have been compared, and the structural gene for the primary/seccndary $A D H$ has been cloned and sequenced. There are open reading frames (ORFs) flanking the adh gene, which may form an operon for solvent production. For the acetoacetyl-CoA reacting enzymes, CoA-transferase and 3-hydroxybutyryl-CoA dehydrogenase have been partially purified, and their structural and catalytic properties will be determined upon purification. A mechanistic understanding of the alternative enzymes and parallel pathways for solvent production will emerge from this and related studies.

\section{Virginia Polytechnic Institute and State University Blacksburg, VA 24061} 163. Enzymological Studies of One-Carbon Reactions in the Pathway of Acetate Utilization
by Methanogenic Bacteria

J.G. Ferry, Department of Anaerobic Microbiology $\quad \$ 183,000$ (FY 90 Funds/2 years)

Several enzymes in the pathway of acetate conversion to methane and carbon dioxide have been purified from Methanosarcina thermophila. The mechanisms of these enzymes are under iivestigation utilizing biochemical, biophysical and molecular genetic approaches. Acetate kinase and phosphotransacetylase catalyzes the activation of acetate to acetyl-CoA. The primary structure of these enzymes will be determined through cloning and sequencing of the genes. Two protein components of the $\mathrm{CO}$ dehydrogenase complex are under investigation. Initial results indicate that the $\mathrm{Ni} / \mathrm{Fe}$-S component cleaves the $\mathrm{C}-\mathrm{C}$ and $\mathrm{C}$-S bonds of acetyl-CoA followed by oxidation of the carbonyl group to carbon dioxide and transfer of the methyl group to the Co/Fe-S component. The metal centers of each component will be further characterizec', using EPR. Cloning and sequencing of the genes for the two subunits of each component is in progress. The enzymes and cofactors involved in transfer of the methyl group from the Co/Fe-S component to coenzyme $M$ will be purified and characterized. Ferredoxin is an electron acceptor for the $\mathrm{Ni} / \mathrm{Fe}-\mathrm{S}$ component and also serves to reductively reactivate methylreductase which catalyzes the demethylation of methyl coenzyme $M$ to 
methane. This ferredoxin is being characterized utilizing EPR and RR spectroscopic methods to determine the properties of the Fe-S centers. Genes encoding this and other ferredoxins are being cloned and sequenced to determine the primary structures. Carbonic anhydrase is being purified and characterized to determine the function of this enzyme in the pathway.

\section{Washington State University Pullman, WA 99164-6340}

\section{Regulation of Terpene Metabolism}

R. Croteau, Institute of Biological Chemistry

$\$ 182,500$ (2 years)

Terpenoid oils, resins, and waxes from plants are important renewable resources. The objective of this project is to understand the regulation of terpenoid metabolism using monoterpenes $\left(C_{10}\right)$ as a model. The focus of the project is the biochemical, cellular, and molecular characterization of $(+)$ camphor metabolism in sage and (-)-menthone metabolism in mint. The pathways of biosynthesis and catabolism have been established, the rate-limiting step of each process has been identified, and the relevant enzymes have been isolated, characterized, and partially purified. Developmental studies relating enzyme activities to terpene production within, and loss from, the oil gland sites of metabolism indicate that monoterpene accumulation is controlled by the balance between the rate-limiting terpene cyclases and the catabolic enzymes responsible for the synthesis of terpenyl glycoside transport derivatives. These key metabolic enzymes are being purified to homogeneity in order to obtain polyclonal antibodies and amino acid sequence information for the preparation of CDNA clones from gland-specific transcripts. Intercellular and intracellular location of the target biosynthetic and catabolic enzymes will be deduced by combination of subcellular fractionation methods and immunocytochemical techniques. Immunochemical methods will also be utilized to determine the temporal levels of the key enzymes and their translatable messages, and the corresponding CDNA clones will be employed to examine transcriptional and translational control of metabolism in greater detail. Results from this project will have important consequences for the yield and composition of terpenuid natural products that can be made available for industrial exploitation.

\section{Washington State University Pullman, WA 99164-6340}

\section{Towards a Detailed Understanding of Structural Variability in Lignins N.G. Lewis, Institute of Biological Chemistry}

Lignins are plant cell wall polymers found in all higher land-based vascular plants. In spite of their abundance, little is known about regulation of lignin formation and its structure in situ. We shall examine three knowledge-deficient areas in lignification, namely, the identity (and the mechanism of transport) of the monomer being transported from the cytoplasm into the lignifying cell wall, lignin structural heterogeneity at both the subcellular level and in different tissue types, and the structure of lignins induced as a response to wounding/fungal attack. In the case of monomer transport, the actual species being exported from the cytoplasm will be identified, e.g., by immunocytochemical techniques, as well as the subcellular location of key enzymes. The role of Z-monolignols in lignification will be established unambiguously. Lignin structural heterogeneity will be examined in two model systems: Pinus taeda cell suspension cultures (an early stage of lignification) and compression wood from Pinus raeda plants. Third, lignin bonding environments and monomer composition of wound/pathogen induced lignins will be determined using solid/solution state carbon-13 NMR spectroscopy. 


\section{Washington State University Pullman, WA 99164-4660}

166. Isocitrate Lyase and the Glyoxylate Cycle

B.A. McFadden, Department of Biochemistry and Biophysics

Our objectives are to shed light upon the structure, regulation and catalytic function of isocitrate lyase (ich), an enzyme which catalyzes the first unique step in the glyoxylate cycle. In this cycle, lipids are converted to carbohydrates.

We have described the cloning and sequencing of the icl gene of Escherichia coli and markedly improved purifications of $i c /$ from E. coli and watermelon. In the present project period, studies of the kinetic mechanism for the $E$. coli enzyme have established that the binding of glyoxylate precedes that of succinate [Current Microbiol. 21, 313 (1990)]. We have also characterized the glyoxylate- and succinate-binding domains of $i c /$ and have alkylated the active-site residue, cys-195. The latter, using the substrate analog brompyruvate, results in marked resistance to proteolysis [Arch.Biochem. Biophys. $278,373(1990)$ ]. We have obtained diffraction-quality crystals of both icl and pyruvyl ic/ [J.Mol.Biol., in press] and continue to work on both crystal structures. His-266 and -306 and ser-319 and -321 have been placed in the active site of $E$. coli icl. Mutagenesis of the gene is being directed towards replacing these and other potentially functional residues to test our hypothesized catalytic mechanism [Comparative Biocriem. 95B, 431 (1991)]. Inferences will be compared with our developing knowledge of the crystal structure for icl.

These studies will provide basic information about $i c l$. The function of this enzyme is vital to microbial growth (on acetate or fatty acids) and to the growth of varied plant seedlings and their subsequent utilization of solar energy.

\section{Washington State University Pullman, WA 99164-6340}

\section{Enhancement of Photoassimilate Utilization by manipulation of the ADPglucose Pyrophosphorylase Gene T.W. Okita, Institute of Biological Chemistry \\ $\$ 145,000$ (FY 90 Funds/2 years)}

Starch synthesis is controlled in large part by the gene activation and expression of ADPglucose pyrophosphorylase as well as the allosteric control of the enzyme activity by 3-phosphoglycerate and $\mathrm{Pi}$. In an effort to increase the conversion of photosynthate into starch and hence, overall plant productivity, we are currently pursuing studies to determine the structure-function relationships of the enzyme subunits and the molecular basis for the control of gene expression of ADFglucose pyrophosphorylase during development of reserve tissues. The temporal expression of the small subunit gene and for sucrose synthase as well as their respective enzyme activities were evaluated during tuber development. mRNA transcripts and enzyme activity levels of sucrose synthase were coincident $i 0$ the patterns of ADPgiucose pyrophosphorylase during tuber development suggesting that these genes may be under similar controls. The primary sequence of the encoded small subunit is highly homologous to the counterparts observed in rice endosperm and spinach leaf, indicating that this gene is highly conserved among different plant species. In collaborative studies with G. Berry (Monsanto Corp.) the small subunit has been expressed in $E$. coli at $1-2 \%$ of total protein. The expressed protein does not, however, complement, a bacterial strain defective for the native enzyme activity. Lastly, cDNA clones for the pyrophosphorylase large subunit have been obtained. The expression of both subunits in bacterial cells will facilitate studies to elucidate the role of each subunit type in allosteric regulation and catalysis. 


\section{Washington University St. Louis, MO 63130}

168. Processing and Targeting of the Thiol Protease, Aleurain J.C. Rogers, Department of Biology

$\$ 178,000$ (FY 90 Funds/2 years)

We are studying an unusual thiol protease, aleurain, that is the product of a single-copy gene in barley. The mRNA is expressed in aleurone cells, where it is increased by treatment with gioberellic acid and decreased by treatment with abscisic acid; in contrast, it is apparently expressed constitutively in leaf and root tissues. Aleurain is synthesized as a proenzyme that is cleaved in two steps after it reaches an acidified post-Golgi compartment; its final destination is a unique type of vacuole in aleurone cells. The sequence of aleurain is $65 \%$ identical to that of mammalian cathepsin $\mathrm{H}$, a lysosomal enzyme of unknown function. We have purified aleurain to homogeneity and find that its $\mathrm{Km}$ for certain synthetic substrates is essentially the same as that of cathepsin $\mathrm{H}$; in addition, aleurain has the same unusual minichain/heavy chain structure found in cathepsin $\mathrm{H}$. Using electroporation of tobacco suspension culture protoplasts, we have expressed aleurain and a secreted barley thiol protease, EP-B; aleurain is properly targeted and processed intracellularly, while EP-B is secreted from the protoplasts. Using fusions made between the two different proteins, we have found that the $\mathrm{N}$-terminai $\sim 25$ amino acids of the aleurain prosequence is sufficient to target EP-B to an iniracellular destination, while the $\mathrm{N}$-terminal 25 amino acids of EP-B cause aleurain to be secreted. Work is underway to identify the minimum number of residues that are sufficient to target a secreted protein to a post-Golgi acidified compartment.

\section{Washington University St. Louis, MO 63130}

169. Hydroxyproline-rich Glycoproteins of the Plant Cell Wall J.E. Varner, Biology Department

The cell walls of plants contain a number of proteins having repetitive sequences, e.g., hydroxyprolinerich glycoproteins, proline-rich proteins, and glycine-rich proteins. These are presumed to have structural roles in the determination of cell wall architecture. We have examined the tissue specific expression of the genes for GRPs and HRGPs in soybean stems and petioles and the accumulation of the corresponding proteins. In young soybean stems, HRGPs are expressed most heavily in cambium cells, in a few layers of cortex cells surrounding primary phloem, and in some parenchyma cells around the primary xylem, whereas GRPs are highly expressed in the primary xylem and also in the primary phloem. In older soybean stems, HRGP genes are expressed exclusively in cambium cells and GRP genes are most heavily expressed in newly differentiated secondary xylem cells. Similar expression patterns of HRGPs and of GRPs were found in soybean petioles and young hypocotyls. Soluble HRGPs are abundant in young hypocotyl apical regions and young root apical regions, whereas in hypocotyl and root mature regions, soluble HRGPs are found only in a few layers of cortex cells surrounding the vascular bundles. GRPs are specifically localized in primary xylem cell walls of young root. These results show that the gene expression of HRGPS and GRPS is developmentally regulated in a tissue-specific manner. In soybean tissues, HRGPs are most heavily expressed in meristematic cells and in some of those cells that may be under stress, whereas GRPs are expressed in all cells that are or are going to be lignified. 


\section{University of Wisconsin Madison, WI 53706}

170. Biochemical and Molecular Analysis of a Transmembrane Protein Kinase from
Arabidopsis thaliana
A.B. Bleecker, Department of Botany

Receptor protein kinases on the plasma membrane of cells interact with various growth factors and hormonal agents, generating intracellular signals from these external signals. As such the recepturs play a pivotal role in the ability of a cell to interact with and respond to its environment. A measure of their importance to normal growth and development is the observation that in animal cells many oncogene products are derived from receptor protein kinases.

We have isolated genomic and cDNA clones encoding a novel receptor-like protein kinase from the higher plant Arabidopsis thaliana. This kinase is being studied by combining biochemical, molecular and genetic apprcaches. Expression of the kinase gene in plants is being examined using Northern blots and domain-specific antibodies. Examining various tissue types yields a general idea as to gene expression; cytochemical analysis should give more explicit information on localization. The kinase is also being purified from plants. The purified kinase will be characterized biochemically in terms of kinase activity, reaction characteristics, and activation by dimerization, allowing us to determine how closely this protein fits the canonical model of a receptor protein kinase. Using a variety of means, potential ligands and substrates for the kinase will also be identified. In addition transgenic plants overexpressing or carrying altered forms of the protein kinase gene are being produced. These experiments will help determine the natural role the kinase plays in a pathway of signal transduction.

\section{University of Wisconsin Madison, WI 53706}

\section{Enzymology of Biological Nitrogen Fixation}

R.H. Burris, Department of Biochemistry

$\$ 94,433$ (FY 90 Funds/2 years)

An organic acid extracted from purified dinitrogenase isolated from a nifV mutant of Klebsiella pneumoniae has been identified as citric acid, whereas the dinitrogenase from the wild type enzyme carries homocitric acid. Evolution of $\mathrm{H}_{2}$ by the nifV mutant is partially inhibited by $\mathrm{CO}$, whereas that by the wild type is not. The activities of each enzyme decreased dramatically below pH 6.2. The control of nitrogenase activity in Azospirillum spp. by the " $\mathrm{NH}_{4}{ }^{+}$switch-off/on" process clearly is posttranslational. The dinitrogenase reductase activating glycohydrolase (DRAG) in A. lipoferum is associated with the cell membranes, and the ADP ribosylation control of dinitrogenase reductase in A. lipoferum is very similar to the system studied extensively in Rhodospirillum rubrum. A genomic library of $A$. lipoferum was constructed, and the genes encoding dinitrogenase reductase ADP-ribosyl transferase (DRAT) and DRAG were cloned. As in $R$. rubrum, draT is located between draG and nifH, the gene encoding dinitrogenase reductase. Nitrogenase activity in $R$. rubrum decreased rapidly in the dark for a period of 40 minutes and then recovered almost completely in 20 minutes in the light, and these light-dark/active-inactive cycles could be repeated. A draT mutant did not exhibit such a cycling response. Tests in the field with ${ }^{15} \mathrm{~N}$ as a tracer demonstrated a marked difference among 21 lines of beans (Phaseolus vulgaris) in their ability to fix $\mathrm{N}_{2}$. There also was a clear difference in the ability of different strains of beans to transfer the fixed nitrogen to tile seeds. 


\section{University of Wisconsin Madison, WI 53706}

172. Molecular Genetics of Ligninase Expression

D. Cullerl and T.K. Kirk, Department of Bacteriology

$\$ 69,000$ (13 months)

Lignin depolymerization is catalyzed by extracellular peroxidases of white rot basidiomycetes such as Phanerochaete chrysosporium. In submerged culture, multiple isozymes of lignin peroxidase (LiP) are secreted at relatively low levels, and production is derepressed under carbon, nitrogen, or sulfur limitation. Our objectives are to elucidate the organization/regulation of the genes encoding LiP's of $P$. chrysosporium and to investigate their expression in Aspergillus nidulans. Toward these goals, we have cloned and sequenced six closely related LiP genes. Three of these genes are clustered within a 30 kilobase region of the geiıme. Further, we have separated the $P$. chrysosporium chromosomes using alternating field electrophoresis and shown that all six LiP genes are present on one dimorphic chromosome. Allelic relationships among gene clusters have been established and detailed physical maps of the LiP-containing chromosomes are being prepared. Specialized primer extension methods have been developed which permit quantitative detection of allele-specific transcripts. The gene encoding LiP isozyme $\mathrm{H} 8$ has been expressed in Aspergillus under the control of the glucoamylase promoter. The long term goal, production of highly purified recombinant peroxidases, may aid in the development of processes such as biological bleaching of pulps, effluent treatments, and in biopulping.

\section{University of Wisconsin Madison, WI 53706}

173. Role of Transit Peptide in the Localization of Nuclear-encoded Chloroplastic Proteins K. Keegstra, Department of Botany

$\$ 95,000$

Most chloroplast proteins are encoded in the nucleus and synthesized in the cytoplasm as precursors containing additional amino acids called a transit peptide. The precursors are post-translationally imported into chloroplasts and segregated to their proper location. The objective of our work is to understand the role of the transit peptide and other topogenic sequences in directing the import and sorting processes. These processes are being studied in an in vitro reconstituted system. In this system, radioactive precursor proteins are synthesized by in vitro expression of cloned precursor genes and the resulting precursor proteins imported into isolated intact chloroplasts. The localization of imported proteins is examined by chloroplast fractionation studies. Genes for precursor proteins destined for the chloroplast envelope membranes have been isolated and their transport pathway is being characterized. With genes for these precursors now available, we are able to study the targeting of proteins to 5 different locations within chloroplasts: outer envelope membrane, inner envelope membrane, stroma, thylakoid membrane and thylakoid lumen. The role of the transit peptides is being examined by generating hybrid precursor proteins containing thie iransit: peptide from a precursor destined for one location fused to the mature peptide destined for a different location. 


\section{University of Wisconsin Madison, WI 53706}

\section{Organization of the $\mathbf{R}$ Chromosome Region in Maize J.L. Kermicle, Laboratory of Genetics}

$\$ 127,000$ (FY 90 Funds/2 years)

Organization of the $R$ region in maize is under study with a view of determining the number, kind, and arrangement of components involved in the control of anthocyarin pigmentation. $R$ is organized on a modular basis and is extensively polymorphic. An allele comprises one or more functionally independent units (genic elements), each distinguished by particular tissue-specific effects. Intragenic recombination serves to place differences between genic elements relative to sites of recessive mutation. Molecular characterization provides detail concerning the structure of regions of particular functional significance. A collection of Dissociation insertion mutations in a seed-coloring ge. ic element is being used to investigate, (1) the relationship of kernel spotting density and germinal mutation spectrum to location of the insert within $R$, and (2) effects of $D s$ inserts on the pattern of intragenic recombination.

\section{University of Wisconsin Madison, WI 53706-1569}

\section{Carbon Monoxide Metabolism by Photosynthetic Bacteria P.W. Ludden and G.P. Roberts, Department of Biochemistry}

This project focuses on the biochemistry, physiology and genetics of the carbon monoxide oxidation system found in the photosynthetic bacterium Rhodospirillum rubrum. The enzyme carbon monoxide dehydrogenase (CODH) is produced by Rhodospirillum rubrum in response to carbon monoxide in the riedium. The enzyme is a nickel and iron sulfur enzyme with a molecular weight of 62,000, and carries out the oxidation of $\mathrm{CO}$ to $\mathrm{CO}_{2}$. The enzyme can be produced in an apo form lacking nickel at the active site; this active site can be filled with $\mathrm{Ni}^{2+}, \mathrm{CO}^{2+}$ or $\mathrm{Fe}^{2+}$ in vitro. Spin labelled substrates such as ${ }^{13} \mathrm{CO}$ and ${ }^{13} \mathrm{CN}$ are being utilized to study the interaction of the ligands to the active site nickel in the enzyme. Enzyme containing ${ }^{61} \mathrm{Ni},{ }^{59} \mathrm{Co}$, or ${ }^{57} \mathrm{Fe}$ at the active site will be analyzed by EPR, ENDOR, Mossbauer and NMR. The gene for CODH has been isolated and sequencing of the gene is near completion. The electron acceptor from $\mathrm{CODH}$ to hydrogenase in vivo has been identified, isolated and characterized as a subunit proteiri that attaches to the $62 \mathrm{kd}$ nickel, iron-sulfur $\mathrm{CODH}$ enzyme. This $22 \mathrm{kd}$ ferredoxin-like protein contains 4 irons in a $\mathrm{Fe}_{4} \mathrm{~S}_{4}$ center and is able to both transfer electrons between $\mathrm{CODH}$ and hydrogenase to bind the enzyme to the membrane.

\section{University of Wisconsin Madison, WI 53706}

176. Starch Synthesis in the Maize Endosperm as Affected by Starch-Synthesizing Mutants O.E. Nelson, Department of Genetics

$\$ 52,315$ (9 months)

In the continuing investigation of the brittle-1 locus (mutant alleles condition a severe block in starch synthesis), a full-length cDNA from a $B t 1$ allele has been cloned and sequenced. The predicted amino acid sequence from this CDNA shows a 75 amino acid leader peptide which contains elements similar to a chloroplast targeting sequence and presumably targets the polypeptide to an amyloplast. The protein also has a stretch with considerable sequence similarity to the nucleotide binding pockets of some mitochondrial membrane proteins raising the possibility that it might serve as an adenylate translocator. There are two putative membrane-spanning domains in the protein. 
We have also investigated a mutant, vitreous*-8132, that identifies a new locus located on the short arm of Chr. 4 that is implicated in starch synthesis. The mutant kernels are slightly shrunken and are $80-85 \%$ as heavy as nonmutant sibs. The developing mutant endosperms have very low activity (ca. $10 \%$ of nonmutant) of a bifunctional enzyme that is capable of cleaving alpha-1, 6-glucose linkages as shown by its ability to attack pullulan with the release of maltotriose. The enzyme can also act as a transferase with the transfer of radioactivity from ${ }^{14} \mathrm{C}$-labelled pullulan to amylose or amylopectin. Thus there are interesting similarities between this enzyme and the glycogen-debranching enzyme from rabbit muscle.

\section{University of Wisconsin Madison, WI 53706}

\section{Induction of Polygalacturonases Important in Plant Pathogenicity of Pseudomonas solanacearum \\ L. Sequeira, Department of Plant Pathology \\ $\$ 76,109$}

The mechanism by which Pseudomonas solanacearum causes bacterial wilt disease of plants is not understood. The purpose of our research is to determine how bacterial production of virulence factors is induced in the presence of the host plant. $P$. solanacearum produces several extracellular polygalacturonases (PG) that degrade plant cell walls. We have determined that the major extracellular PG produced in culture, encoded by pehA, is produced constitutively and is not required for pathogenesis. However, $P$. solanacearum produces two additional PGs whose activity increases in response to plant extracts, nutritional stress, and galacturonic acid (a breakdown product of piant cell walls). Two transposon mutants of $P$. solanacearum, each lacking one of the inducible PGs, are substantially reduced in virulence. Thus, plant-inducible PG activity is an important factor in virulence of this bacterium. The transposon insertions are being used to isolate the corresponding wildtype inducible PG genes. The enzymes encoded by these genes are being characterized and reporter gene insertions in the cloned genes will be used to study the induction profile of each FG gene. We are particularly interested in identifying the plant factor(s) that induce bacterial PG production since they may also induce other previously unideritified genes important in pathogenesis.

\section{University of Wisconsin Madison, WI 53706}

\section{Feedback Limitations of Photosynthesis}

T.D. Sharkey, Department of Botany

$\$ 152,000$ (FY 90 Funds/2 years)

The regulation of photosynthesis in intact leaves is studied by combining analytical gas exchange analyses with biochemical, biophysical, molecular biological, and ultrastructural techniques. The long term goal is to understand how photosynthesis is regulated and how it can be modified.

We demonstrated that the oxygen sensitivity of photosynthesis can be used to determine when starch and sucrose synthesis limit the rate of photosynthesis. This probe was used to find a variant of Flaveria linearis in which photosynthesis is usually limited by the rate of sucrose synthesis. We are currently preparing DNA primers to amplify the gene for cytosolic fructose bisphosphatase (F'BPase) which is known to have a low activity in the variant. When ${ }^{14} \mathrm{CO}_{2}$ is fed to the variant, relatively more carbon becomes starch but also, more carbon enters the acidic amino acid pool. The distribution of starch is being assessed by ultrastructural methods, and the distribution of cytosolic FBPase will be determined by immunolocalization. 
The properties of photosynthesis in a transgenic plant expressing excess phytochrome are under investigation. Leaves of these plants have higher levels of many enzymes of photosynthesis including sucrose synthesizing enzymes. However, the overall rate of photosynthesis in air is lower because of a large resistance to $\mathrm{CO}_{2}$ diffusion in the mesnnisyll. The cause of the increased diffusion limitation is under investigation using stable carbon isotope discrimination analysis.

The mechanism of feedback limitation on electron transport will be studied by non-invasive optical measurements of the status of the photosynthetic electron transport chain.

\section{University of Wisconsin Madison, WI 53706}

\section{Molecular Mechanism of Energy Transduction by Plant Membrane Proteins M.R. Sussman, Department of Horticulture \\ $\$ 206,250$ (2 years)}

Proton pumps are membrane-buund proteins that act as biological energy transducers. The plasma membrane of plants and fungi contains an ATP-coupled proton pump ( $\mathrm{H}^{+}$-ATPase) that converts chemical into electrical energy. This enzyme is essential for the growth of plants and fungi and provides the driving force used to catalyze the uptake and accumulation of solutes. The plant/fungal plasma membrane $\mathrm{H}^{+}$-ATPase contains a single polypeptide of $\mathrm{Mr}=100,000$. Its simple polypeptide structure makes this enzyme an attractive candidate for studying the molecular basis of energy transduction. A major aim of this project is to identify aspects of the enzyme's primary structure that are essential for converting chemical into electrical energy. DNA cloning and sequencing techniques are being used to obtain the complete amino acid sequence for ATPase structural genes present in Arabidopsis thaliana, a higher plant with a small genome and a rapid generation time. The sequence of genomic and CDNA clones is being determined for each of the several ATPase gene isoforms present in the nuclear genome. Expression of these genes is being studied using Northern blots and GUS gene fusions with putative ATPase promoter sequences. These studies on cell-specific expression of the ATPase gene will help to delineate the developmental and environmental signals that regulate activity of the plasma membrane proton pump in situ. In addition, these studies provide data necessary for testing hypotheses concerning the biological role of ion pumps and the molecular mechanism of proiein-mediated energy transduction in plants.

\section{University of Wisconsin Madison, WI 53706}

\section{Mechanism for the Selective Conjugation of Ubiquitin to Phytochrome} R.D. Vierstra, Department of Horticulture

$\$ 186,000$ (2 years)

The selective degradation of intracellular proteins is an important component in the regulation of plant cell physiology and development. The goal of this proposal is to determine how proteins are selectively committed to breakdown by characterizing as a model system ihe selective degradation of the plant photoreceptor chromoprotein, phytochrome. Phytochrome exists in two photointerconvertible forms, a red light-absorbing form, Pr, and a far-red light-absorbing form, Pfr. Because the degradation of $\mathrm{Pfr}$ is approximately 100 times faster than $\mathrm{Pr}$, this systein offers us the unique ability to rapidly and synchronously manipulate the degradation rate of a protein by noninvasive light irradiations. We have previously shown in a variety of plant species that Pfr-specific degradation of phytochrome is accomplished by a proteolytic pathway involving the 76-aa protein ubiquitin. The ubiquitin pathway functions by covalently attaching ubiquitin to proteins destined for catabolism with the ubiquitin moiety then serving as a recognition signal for specific proteases. In an effort to further understand how phytochrome is recognized by the ubiquitin pathway, we have 
exploited a transgenic system involving the expression of native oat phytochrome in tobacco. The oat protein, like its tobacco counterpart, is rapidly conjugated with ubiquitin and degraded after photoconversion to $\mathrm{Pfr}$. This demonstrates that the domains responsible for selective ubiquitination and degradation are conserved betw een the monocot and dicot chromoproteins. Using this transgenic system, we are now attempting to identify regions involved in ubiquitin ligation by site-directed mutagenesis of the oat phytochrome gene and subsequent characterization of the protein expressed in tobacco. Successful completion of this proposal will provide new insights into the molecular mechanisms for selective ubiquitin conjugation and generate new information on the regulation of protein turnover in plants.

\section{Worcester Foundation for Experimental Biology Shrewsbury, MA 01545}

\section{Hovel Biomaterials: Genetically Engineered Pores \\ H. Bayley}

$\$ 78,525$

(Cofunded with the Division of Materials Sciences)

Recombinant DNA technology allows the generation of new materials in microorganisms. We are constructing a selection of microscopic pores by genetic manipulation of a bacterial channel protein. They include: pores with different internal diameters, with selectivity for the passage of molecules and ions, and with gating properties (the ability to open and close in response to a physical stimulus, e.g., an electric field or light). The molecule we are remodeling is a $\alpha$-hemolysin ( $\alpha \mathrm{HL}$ ), which is secreted by Staphylococcus aureus. It is a small robust molecule that should be relatively easy to reengineer. The sinyle polypeptide chain of $33.2 \mathrm{kDa}$ forms hexameric pores in membranes $\sim 11 \AA$ in internal diameter. The hexamer can be assembled from the monomer in vitro by the addition of an inexpensive detergent. Recombinant $\alpha H L(r-\alpha H L)$ has now been obtained in multimilligram amounts by overexpression of the $\alpha \mathrm{HL}$ gene in $E$. coli. $r-\alpha H L$ is identical to $\alpha \mathrm{HL}$ purified from $S$. aureus by several criteria, including its pore-forming properties in artificial lipid bilayers. The pore-forming domain of $\alpha \mathrm{HL}$ is currently being located by deletion mutagenesis. Subsequently, this domain will be altered by point mutagenesis combined with chemical modification to produce new pores with a range of properties. Ultimately, the new pores will be used to confer novel permeability properties upon materials such as thin films. Such products have potential technological applications: for example, as components of energy conversion and storage devices, selective electrodes, electronic devices, and ultrafilters.

\section{Yale University New Haven, CT 06511}

\section{Molecular Cloning and Structural Characterization of the R Locus of Maize} S.L. Dellaporta, Department of Biology

Certain $R$ alleles comprise two genetically distinct components that confer the plant $(P)$ and seed (S) aspects of the pigmentation pattern. These alleles are meiotically unstable, losing $(P)$ or $(S)$ function, often accompanied by exchange of flanking markers. We have shown that the (P) component consists of a single gene within the R-r complex whereas the (S) component is part of a more complex arrangement of multiple $R$ genes or gene subfragments. A third, cryptic region of the complex, termed $(Q)$, has also been cloned and consists of a truncated $R$ sequence. The analysis of $R$-r derivative alleles shows they arise from unequal exchange between the $(P)$ gene and one of several distinct regions of the R-r complex. Restriction site polymorphisms were used to show that most of these unequal exchanges are intragenic. The frequency of displaced intragenic recombination is comparable to previous estimates for intragenic recombination in maize involving genes thai are not duplicated. 
These exchange events have been used to determine the arrangement of components within the complex and their orientation in the chromosome. We also have shown that localized rearrangements in the $(P)$ and $(S)$ components are responsible for non-crossover derivative alleles.

\section{Yale University New Haven, CT 06511-7444}

\section{Spatial Regulation of $\mathrm{C} 4$ Genes in $\mathrm{C} 3, \mathrm{C4}$, and $\mathrm{C} 3 / \mathrm{C} 4$ Intermediate Flaveria Species}

The dicot genus Flaveria (Asteraceae) includes species utilizing C3, C4, and C3/C4 intermediate schemes of carbon fixation. In the efficient $\mathrm{C} 4$ scheme, neighboring photosynthetic bundle sheath (BS) and mesophyll (M) cells cooperate for carbon fixation. $\mathrm{CO}_{2}$ is fixed initially in $\mathrm{C} 4$ acids in $\mathrm{M}$ cells, then further metabolized in BS cells, via a pathway that relies on expression of the genes for pathway enzymes in M- or BS-specific patterns. In less efficient $\mathrm{C} 3$ species, $\mathrm{M}$ cells independently fix $\mathrm{CO}_{2}$ in a C3 compound, and the BS is not generally photosynthetic. C3/C4 intermediate species exhibit anatomical and biochemical characteristics between the $\mathrm{C} 4$ and $\mathrm{C} 3$ extremes and may represent evoluticnary steps between $\mathrm{C} 3$ and $\mathrm{C} 4$ forms. In both $\mathrm{C} 3$ and $\mathrm{C} 3 / \mathrm{C} 4$ species, $\mathrm{C} 4$ pathway genes are present, but used in different spatial patterns. The variety of Flaveria species provides an opportunity to compare the structure and regulation of $\mathrm{C} 4$ pathway genes in closely related $\mathrm{C} 3$ and $\mathrm{C} 3 / \mathrm{C} 4$ species. Our ongoing work includes (1) the isolation and comparison of genes for phosphoenolpyruvate carboxylase (PEPCase) and ribulose bisphosphate carboxylase (RuBPCase) from C3 and C4 species of Flaveria, (2) the characterization of spatial and temporal expression patterns of these genes in $\mathrm{C} 3$, $\mathrm{C} 4$, and $\mathrm{C} 3 / \mathrm{C} 4$ species, and (3) the expression of PEPCase- and RuBPCase-reporter gene fusions in leaf cells of $\mathrm{C} 3, \mathrm{C} 4$ and $\mathrm{C} 3 / \mathrm{C} 4$ species.

\section{Yale University New Haven, CT 06511}

\section{Organization and Control of Genes Encoding Catabolic Enzymes in Rhizobiaceae D. Parke and L.N. Ornston, Department of Biology $\$ 166,000$ (2 years)}

The goal of this project is to understand energy metabolism, gene organization, and gene control in plant-associated bacteria of the family Rhizobiaceae. The group includes the plant pathogen Agrobacterium and the legume symbionts Rhizobium and Bradyrhizobium. Bacteria of the three genera grow at the expense of a wide range of monocyclic phenolics which originate from lignin and plant root exudates. The B-ketoadipate pathway, serving to break down diverse phenolic compounds, is universally distributed in members of the Rhizobiaceae. Inducible in Agrobacterium, Rhizobium, and all other microbes known to possess them, enzymes of the B-ketoadipate pathway are expressed constitutively in Bradyrhizobium. One enzyme in particular, B-ketoadipate succinyl CoA transferase, a product of the pcaE gene, is expressed at high levels in saprophytic and symbiotic Bradyrhizobium. Genes encoding enzymes of the B-ketoadipate pathway from Bradyrhizobium japonicum $1-110$ will be cloned, their genetic organization studied, and the genetic hasis for high and low level expression of different enzymes determined. Mutant strains of $B$. japonicum blocked in the catabolism of phenolics have been isolated and will be used to investigate the selective basis for the constitutive trait in Bradyrhizobium. The molecular genetics of phenolic catabolism in Agrobacterium has been under investigation; further studies of intriguing aspects of regulation in this genus will provide a point of reference for other members of the Rhizobiaceae. Southern hybridization using cloned genes will localize genes for phenolic catabolism to plasmids or the chromosome in diverse representatives of this bacterial family. 


\section{Yale University New Haven, CT 06510}

185. Mechanisms and Control of $\mathbf{K}^{+}$Transport in Plants and Fungi

C.L. Slayman, Department of Cellular and Molecular Physiology

$\$ 107,000$

The overall objective of this research is a detailed functional description of potassium transport in model plant/fungal systems. The detailed objectives of the experiments have become the development of preparations to take advantage of genetics/molecular biology in structure-function studies of $\mathrm{K}^{+}$-transport proteins. The main preparations being examined are the tonoplast (vacuolar membrane) of Saccharomyces cerevisiae and the plasma membranes of Arabidopsis thaliana and of Neurospora crassa. The yeast tonoplast has proven an excellent preparation for patch recording, since it can be released from yeast protoplasts as large, clean, easy-to-patch spheres. The preparation opens the possibility of detailed electrical-kinetic studies on both native transporters and cloned transporters from other sources. Over the next year, we plan to complete description of the major cation channel in the yeast tonoplast, including its calmodulin dependence, physiological substrates, interactions of gating-modulator agents $\left(\mathrm{Ca}^{++}\right.$, membrane potential, redox compounds), and intrinsic conductance characteristics. We will also characterize the primary proton pump of the tonoplast membrane using "whole-vacuole" patch recording. This should be the first V-type ATPase examined systematically by electrical kinetics, with ramifications for understanding both of vacuolar ion transport and of energy metabolism, since V-type ATPases are very closely related to F-type (mitochondrial, chloroplast) ATPases. Finally, we expect to finish writing up four groups of experiments: Temperature dependence of pump/leak coordination in Neurospora; Pitfalls of using lipid-soluble ions for voltage probes in plants and fungi; Enumeration and preliminary description of channels in the Arabidopsis plasma membrane; and Status of partial clones for $\mathrm{K}^{+}$-transporters in Neurospora.

\section{Yale University New Haven, CT 06511}

\section{Transfer RNA Involvement in Chlorophyll Biosyinthesis}

D. Soll, Dept. of Molecular Biophysics and Biochemistry $\$ 198,000$ (FY 90 Funds/2 years)

Chlorophyll is the major pigment in photosynthesis. One molecule of chlorophyll is synthesized from eight molecules of $\delta$-aminolevulinic acid, the universal precursor of porphyrins. Chlorophyll synthesis is regulated at the synthesis of $\delta$-aminolevulinic acid, which is formed in the stroma of greening plastids from glutamate. The mechanism of $\delta$-aminolevulinic acid synthesis from glutamate is not completely understood. There is now solid evidence that in the chloroplasts of plants and green algae, in the cyanobacteria (e.g., Synechocystis 6803) and in some eubacteria (e.g., E. coli and B. subtilis) and archaebacteria this biosynthetic pathway (C5-pathway) involves the reduction of glutamate to glutamate-1-semialdehyde which is subsequently converted to $\delta$-aminolevulinate. It is unclear whether an additional pathway for $\delta$ - aminolevulinic acid synthesis exists in these organisms. The C5-pathway involves tRNA ${ }^{\text {Glu }}$ and three enzymes. In the first step glutamate is attached to the tRNA by the glutamyi-tRNA synthetase. The subsequent reduction of glutamate to glutamate semialdehyde is carried out by Glu-tRNA reductase which requires tRNA as a specific "cofactor". In the final step an aminotransferase converts glutamate-1-semialdehyde to $\delta$-aminolevulinic acid. We have purified the three enzymes from Chlamydomonas and reconstituted the in vitro conversion of glutamate to $\delta$-aminolevulinic acid from three purified enzymes and a purified tRNA. We have also cloned the genes for Glu-tRNA reductase and GSA-aminotransferase from Synechocystis 6803 and Arabidopsis. The goal of our studies is the cloning and characterization of the genes encoding all three enzymes and an understanding of the regulation of this biosynthetic pathway. These studies should uncover novel principles regarding the rcle of tRNA as a cofactor in metabolic conversions. 


\section{INVESTIGATOR INDEX}

Abdelal, A.T. -- Georgia State University . . . . . . . . . . . . . . . . . . . 54

Adams, M.W.W -- University of Georgia . . . . . . . . . . . . . . . . . . . . . . 55

Albersheim, P. .- University of Georgia . . . . . . . . . . . . . . . . . . . . . . . . . . . . . . . . . . . . . . . . . . . .

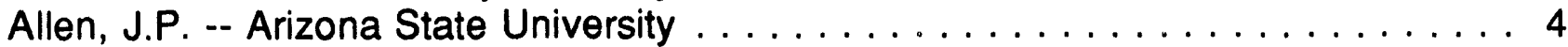

Alper, M.D. -- Lawrence Berkeley Laboratory . . . . . . . . . . . . . . . . . . . 86

Arp, D.J. -- Oregon State University . . . . . . . . . . . . . . . . . . . . . . 137

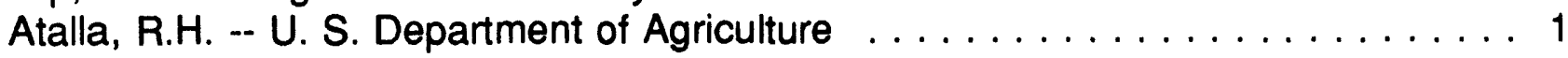

Barkan, A. -- University of Oregon . . . . . . . . . . . . . . . . . . . . 139

Bayley, H. -- Worcester Foundation for Experimental Biology . . . . . . . . . . . . 181

Beachy, R.N. -- Scripps Clinic \& Research Foundation . . . . . . . . . . . . . . . 154

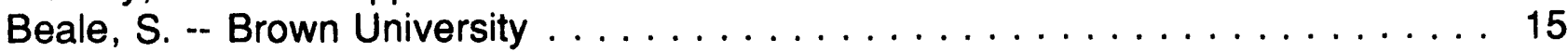

Bednarski, M. -- Lawrence Berkeley Laboratory . . . . . . . . . . . . . . . . . . 86

Blake, R., II .- Meharry Medical College . . . . . . . . . . . . . . . . . . . . 99

Blanch, H.W. -- Lawrence Berkeley Laboratory . . . . . . . . . . . . . . . . . . . 86

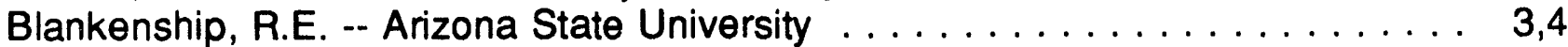

Blanton, R.L. -- Texas Tech University . . . . . . . . . . . . . . . . . . . . . 160

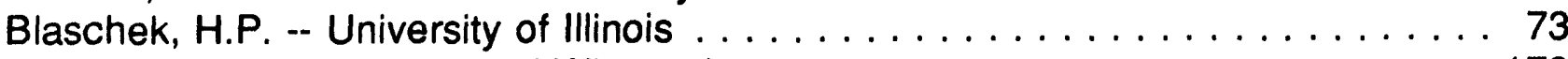

Bleecker, A.B. -- University of Wisconsin . . . . . . . . . . . . . . . . . . 170

Bogorad, L. -- Harvard University . . . . . . . . . . . . . . . . . . . 70

Boyer, P.D. .- University of California . . . . . . . . . . . . . . . . . . . . . 29

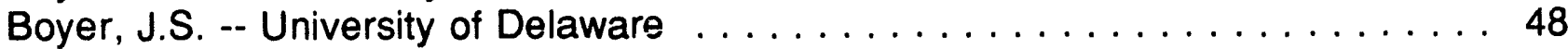

Briskin, D.P. -- University of Illinois . . . . . . . . . . . . . . . . . . . 74

Bruening, G. .- University of California . . . . . . . . . . . . . . . . . . . . 24

Burr, B. -- Brookhaven National Laboratory . . . . . . . . . . . . . . . . . . . 11

Burr, F.A. -- Brookhaven National Laboratory . . . . . . . . . . . . . . . . . . . 11

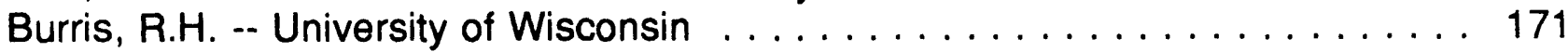

Burton, G.W. .- University of Georgia . . . . . . . . . . . . . . . . . . . . . . 69

Canale-Parola, E. -- University of Massachusetts . . . . . . . . . . . . . . . 97

Carpita, N.C. -- Purdue University . . . . . . . . . . . . . . . . . . . . . . 146

Cashmore, A.R. -- University of Pennsylvania . . . . . . . . . . . . . . . . . 143

Chen, J.-S. -- Virginia Polytechnic Institute and State University . . . . . . . . . . . 162

Cheniae, G.M. -- University of Kentucky . . . . . . . . . . . . . . . . . . . . . 84

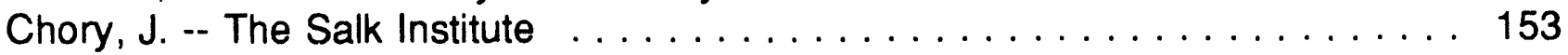

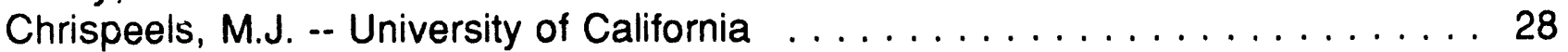

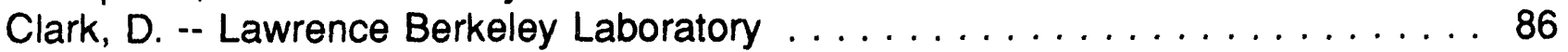

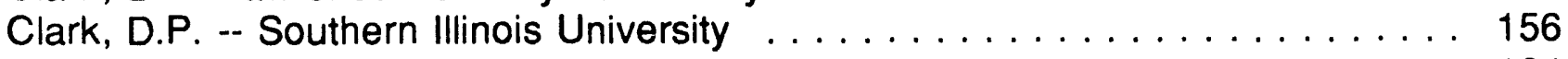

Conway, T. -- University of Nebraska . . . . . . . . . . . . . . . . . . . . 124

Coruzzi, G. -- The Rockefeller University . . . . . . . . . . . . . . . . . . . . . . 149

Cosgrove, D.J. -- Pennsylvania State University . . . . . . . . . . . . . . . . . 140

Crawford, D.L. - University of Idaho . . . . . . . . . . . . . . . . . 71

Cregg, J.M. -- Oregon Graduate Institute of Science and Technology . . . . . . . 135

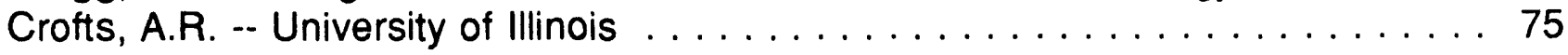


Croteau, R. -- Washington State University . . . . . . . . . . . . . . . . . 164

Cullen, D. -- University of Wisconsin . . . . . . . . . . . . . . . . . . 172

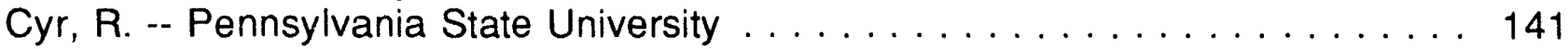

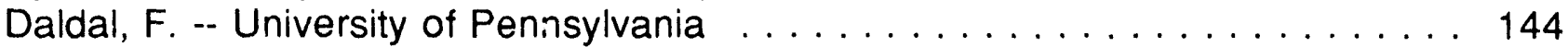

Daniels, C.J. -- Ohio State University . . . . . . . . . . . . . . . . . . . . . 127

Darvill, A. -- University of Georgia . . . . . . . . . . . . . . . . . . . 5 57,59

de Bruijn, F.J. -- Michigan State University DOE Plant Research Laboratory . . . 101

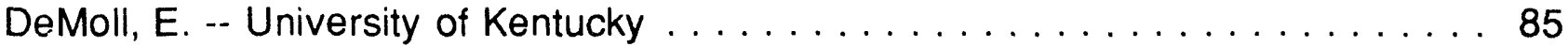

Dellaporta, S.L. -- Yale University . . . . . . . . . . . . . . . . . . . 182

Jelmer, D. -- Michigan State University DOE Plant Research Laboratory . . . . . 102

Dilley, R.A. -- Purdue University . . . . . . . . . . . . . . . . . . . . . . . . 147

Dougherty, W. -- Oregon State University . . . . . . . . . . . . . . . 138

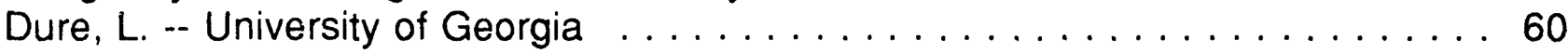

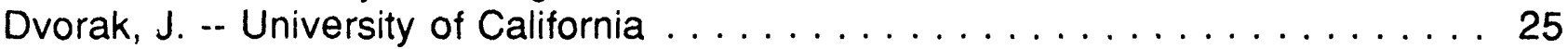

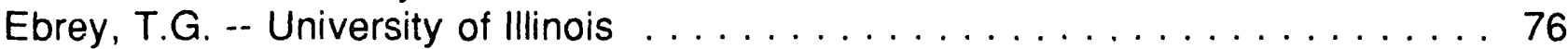

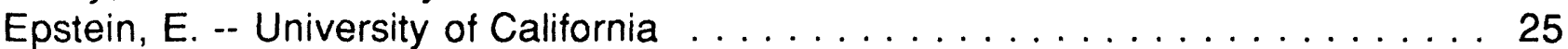

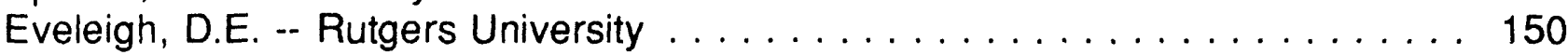

Falkowski, P.G. -- Brookhaven National Laboratory . . . . . . . . . . . . . . . 12

Ferry, J.G. -- Virginia Polytechnic Institute and State University . . . . . . . . 163

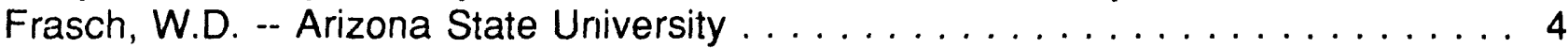

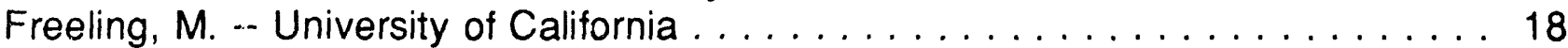

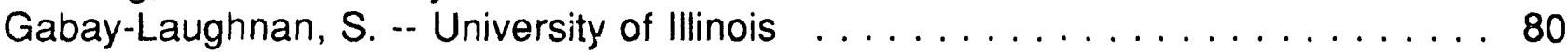

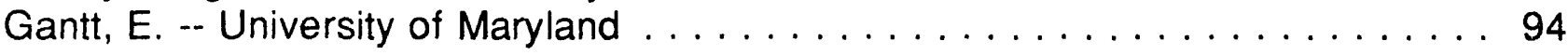

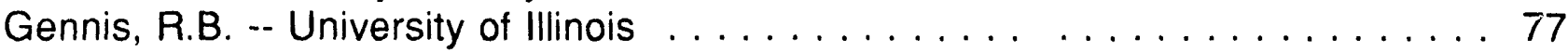

Gibbs, M. -- Brandeis University . . . . . . . . . . . . . . . . . . . . 10

Gibson, J. -- Cornell University . . . . . . . . . . . . . . . . . . . . . 38

Gold, M.H. -- Oregon Graduate Institute of Science and Technology . . . . . 134,135

Grainger, D.W. -- Oregon Graduate Institute of Science and Technology . . . . 136

Green, P. -- Michigan State University DOE Plant Research Laboratory . . . . . . 103

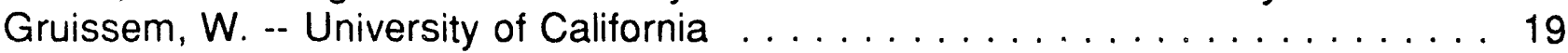

Guerinot, M.L. -- Dartmouth College . . . . . . . . . . . . . . . . . . . . 47

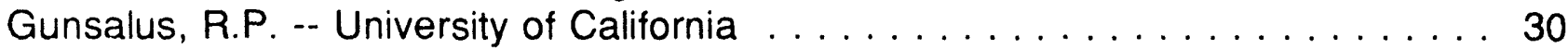

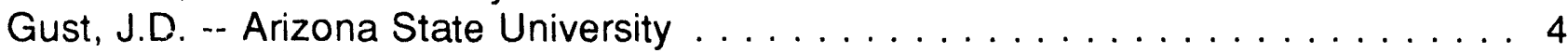

Hanna, W.W. -- University of Georgia . . . . . . . . . . . . . . . . . . 69

Hanson, R.S. -- University of Minnesota . . . . . . . . . . . . . . . . . . . . 120

Hanson, A.D. -- Michigan State University DOE Plant Research Laboratory . . . . 104

Hanson, M.R. -- Cornell University . . . . . . . . . . . . . . . . . . . . . 39

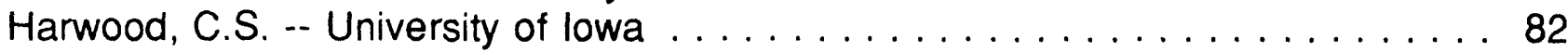

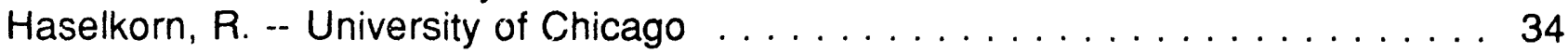

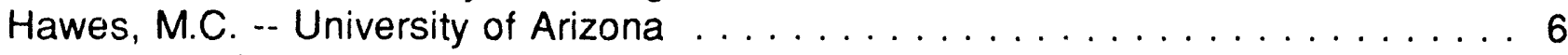

Hearst, J. .- Lawrence Berkeley Laboratory . . . . . . . . . . . . . . . . . . . . 87

Hind, G. -- Brookhaven National Laboratory . . . . . . . . . . . . . . . . . 13

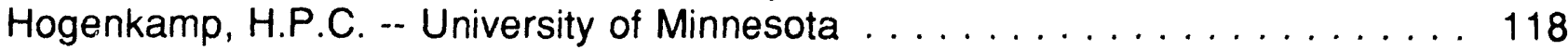

Hollingsworth, R.I. -- Michigan State University . . . . . . . . . . . . . . . . 115

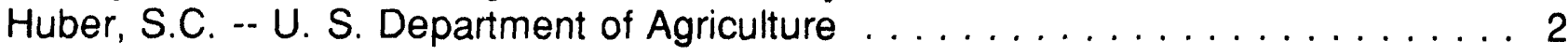




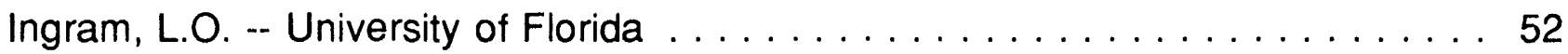

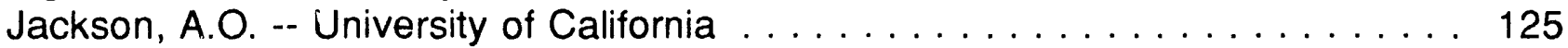

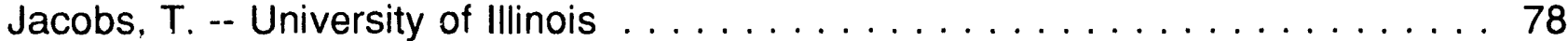

Jair, M.K. -- Michigan Biotechnology Institute . . . . . . . . . . . . . . . 100

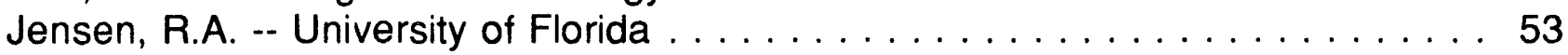

Keegstra, K. University of Wisconsin . . . . . . . . . . . . . . . . 173

Kende, H. -- Michigan State University DOE Plant Research Laboratory . . . . . . 105

Kermicle, J.L. -- University of Wisconsin . . . . . . . . . . . . . . . . . . . . 174

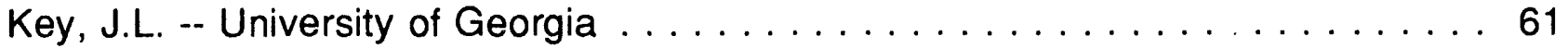

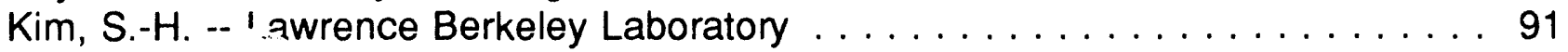

Kirk, T.K. -- Uliversity of Wisconsin . . . . . . . . . . . . . . . . . . . 172

Kirsch, J.F. -- Lawrence Berkeley Laboratory . . . . . . . . . . . . . . . . . 86

Klein, M.P. -- Lawrence Berkeley Laboratory . . . . . . . . . . . . . . . . . . 88

Knaff, D.B. -- Texas Tech University . . . . . . . . . . . . . . . . . . 161

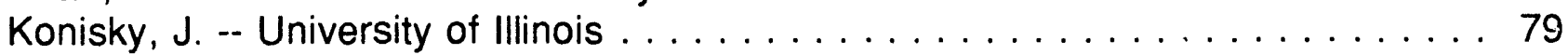

Krulwich, T.A. - Mount Sinai School of Medicine . . . . . . . . . . . . . . 123

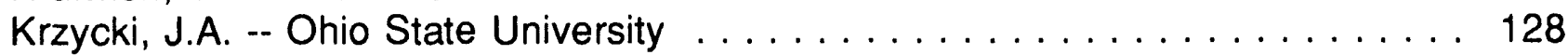

Kuchka, M. -- Lehigh University . . . . . . . . . . . . . . . . . . . . . . . 92

Lagrimini, L.M. -- Ohio State University . . . . . . . . . . . . . . . . . . . . 129

Lamport, D.T.A -- Michigan State University DOE Plant Research Laboratory . . . 106

Lanyi, J.K. -- University of California . . . . . . . . . . . . . . . . . . . 27

LaRoche, J. -- Brookhaven National Laboratory . . . . . . . . . . . . . . . . . . . 12

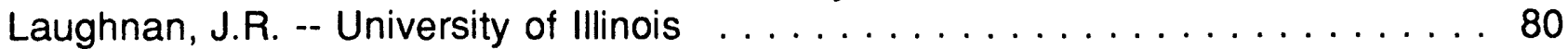

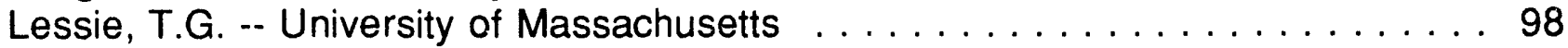

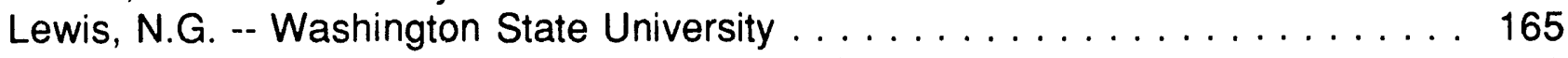

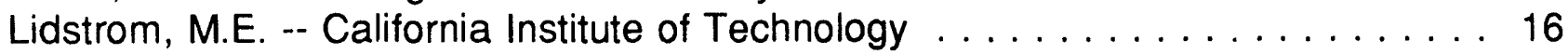

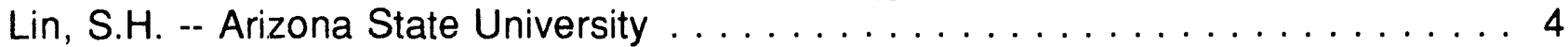

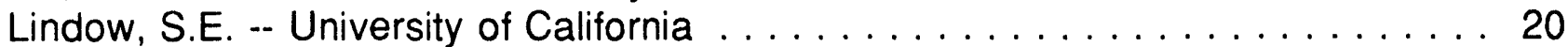

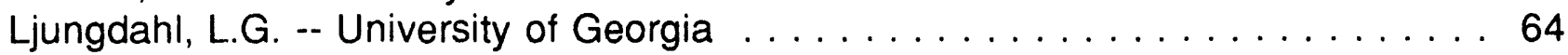

Lonig, S.R. -- Stanford University . . . . . . . . . . . . . . . . . . . . . 157

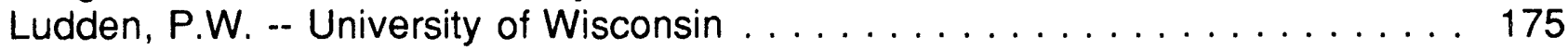

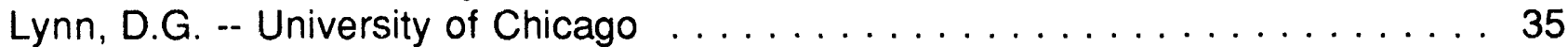

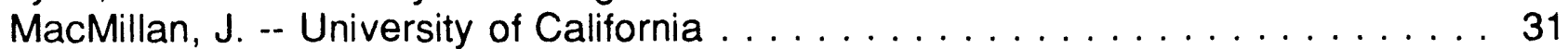

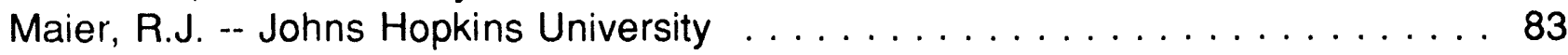

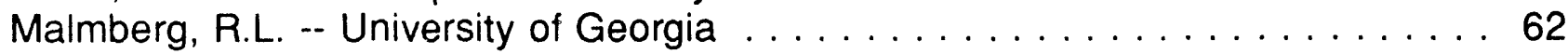

Martienssen, R. -- Cold Spring Harbor Laboratory . . . . . . . . . . . . . . . . . 37

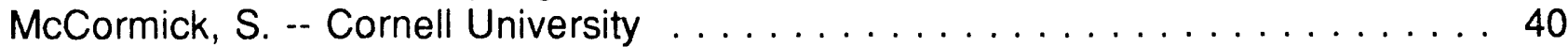

McFadden, B.A. -- Washington State University . . . . . . . . . . . . . 166

Mclnerney, M.J. -- University of Oklahoma . . . . . . . . . . . . . . . . . . 133

McIntosh, L. -- Michigan State University DOE Plant Research Laboratory . . . . 107

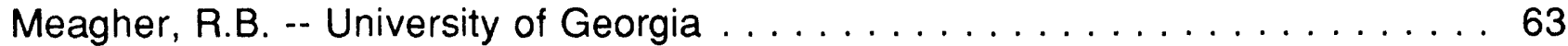

Messing, J. -- Rutgers University . . . . . . . . . . . . . . . . . . . . . . . 152

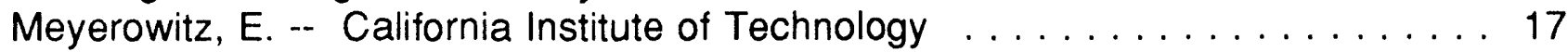

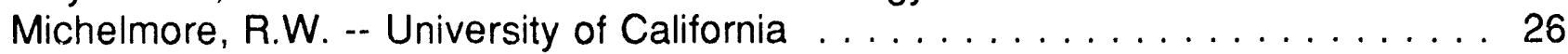

Moore, A.L. -- Arizona State University . . . . . . . . . . . . . . . . . . 4 


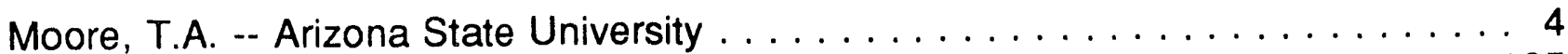

Morris, T.J. -- University of Nebraska . . . . . . . . . . . . . . . . . . . . . 125

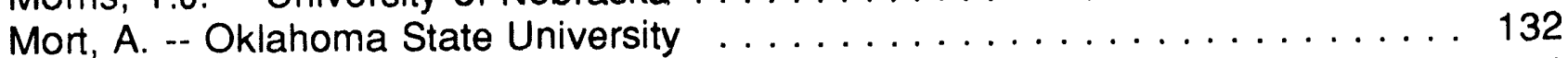

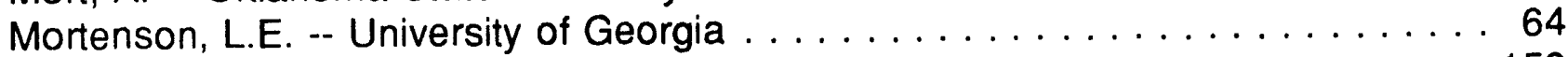

Mullet, J.E. - Texas A\&M University . . . . . . . . . . . . . . . . . . . . . . 159

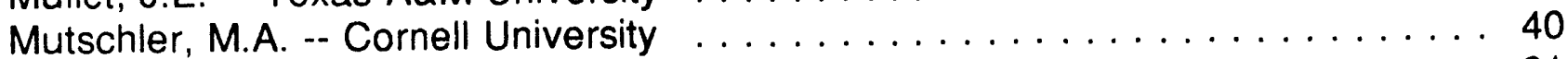

Nagao, R.T. -- University of Georgia $\ldots \ldots \ldots \ldots \ldots \ldots \ldots \ldots \ldots \ldots \ldots$

Nasrallah, J.B. -- Cornell University . . . . . . . . . . . . . . . . . 41

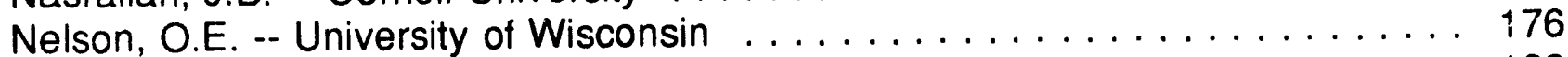

Nelson, T. -- Yale University . . . . . . . . . . . . . . . . . . . . . . 183

Newton, K.J. -- University of Missouri . . . . . . . . . . . . . . . . . 121

Ohlrogge, J.B. -- Michigan State University . . . . . . . . . . . . . . . . 116

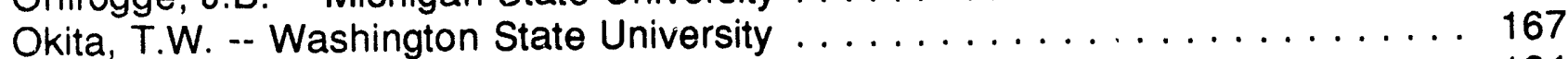

Ornston, L.N. -- Yale University . . . . . . . . . . . . . . . . . . . . . . . . . . . 184

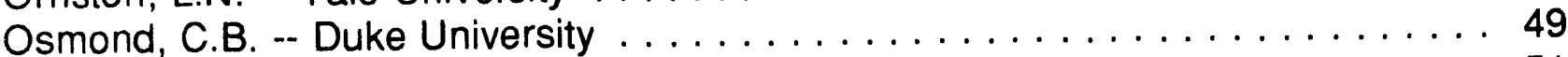

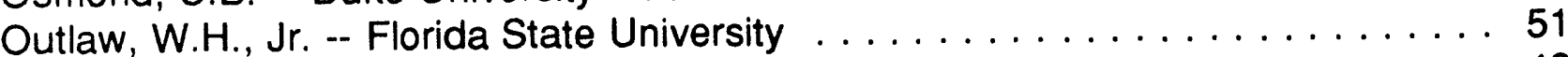

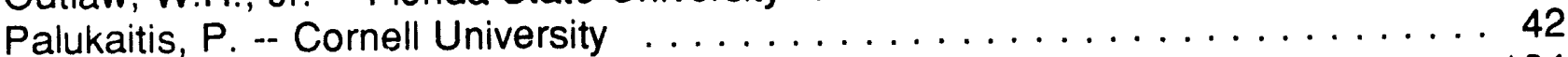

Parke, D. - Yale University . . . . . . . . . . . . . . . . . . . . . . 184

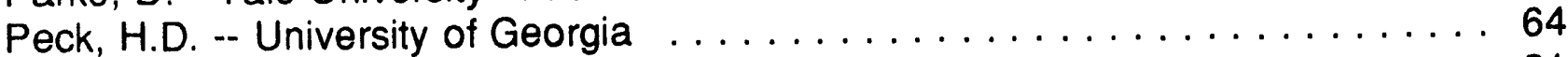

Phinney, B.O. -- University of California . . . . . . . . . . . . . . 31

Poff, K.L. -- Michigan State University DOE Plant Research Laboratory . . . . . . 108

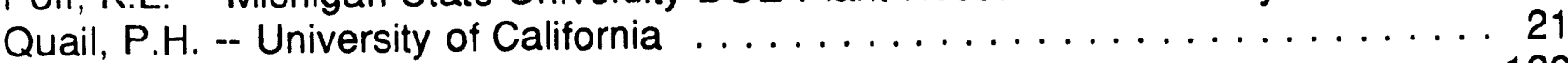

Ragsdale, S.W. -- University of Nebraska . . . . . . . . . . . . . . . . . . 126

Raikhel, N.V. -- Michigan State University DOE Plant Research Laboratory . . . 109

Rananavare, S.B. -- Oregon Graduate Institute of Science and Technology . . . 136

Rapoport, H. -- Lawrence Berkeley Laboratory . . . . . . . . . . . . . . . . . . . . 89

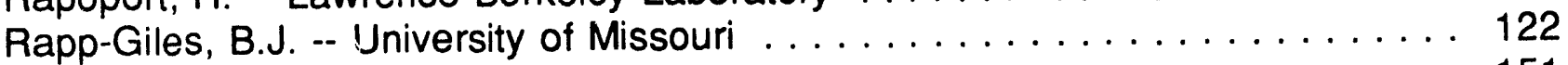

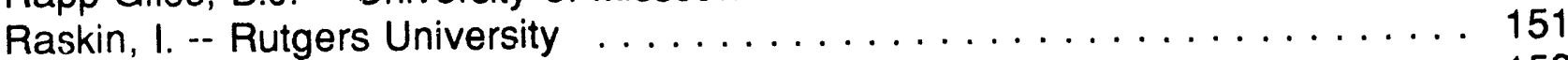

Ray, P.M. -- Stanford University . . . . . . . . . . . . . . . . . . 158

Rea, P.A. -- University of Pennsylvania . . . . . . . . . . . . . . . . 145

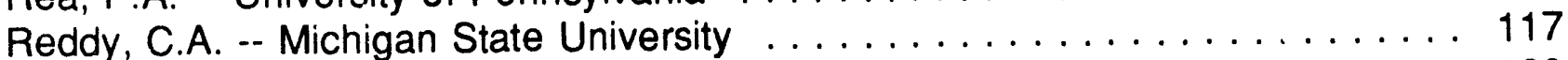

Reeve, J.N. -- Ohio State University . . . . . . . . . . . . . . . . . . . . 130

Renganathan, V. -- Oregon Graduate Institute of Science and Technology . . . . 135

Roberts, G.P. -- University of Wisconsin . . . . . . . . . . . . . . . . . 175

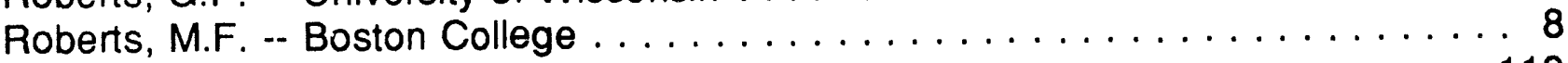

Rogers, P. -- University of Minnesota . . . . . . . . . . . . . . . . . 119

Rogers, J.C. -- Washington University . . . . . . . . . . . . . . . . 168

Sachs, M.S. -- Oregon Graduate Institute of Science and Technology . . . . . . 135

Sauer, K. -- Lawrence Berkeley Laboratory . . . . . . . . . . . . . . . . . . . . . 90

Schmidt, G.W. -- University of Georgia . . . . . . . . . . . . . . . . . 65

Schultz, P.G. -- Lawrence Berkeley Laboratory . . . . . . . . . . . . . . . 86

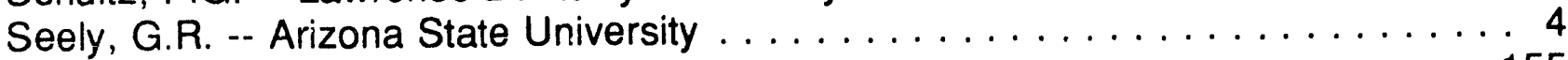

Seibert, M. -- Solar Energy Research Institute . . . . . . . . . . . . . . . 155

Sequeira, L. -- University of Wisconsin . . . . . . . . . . . . . . . . . . . 177

Sharkey, T.D. -- University of Wisconsin . . . . . . . . . . . . . . . . 178 


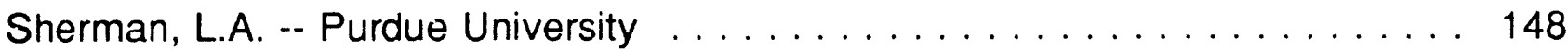

Siedow, J.N. -- Duke University . . . . . . . . . . . . . . . . . . . . . . 50

Siegelman, H.W. .- Brookhaven National Laboratory . . . . . . . . . . . . . . . . . 14

Silver, 3. -- University of Illinois . . . . . . . . . . . . . . . . . . . . . . . . 72

Silverberg, M. -- Oregon Graduate Institute of Science and Technology . . . . . . 136

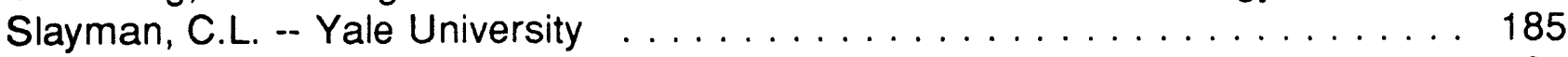

Soll, D. -- Yale University . . . . . . . . . . . . . . . . . . . . . . . . 186

Somerville, C.R. -- Michigan State University DOE Plant Research Lab. . . . . . . 110

Somerville, S.C. -- Michigan State University DOE Plant Research Lab. . . . . . . 111

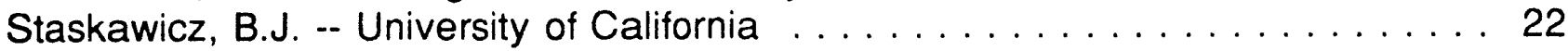

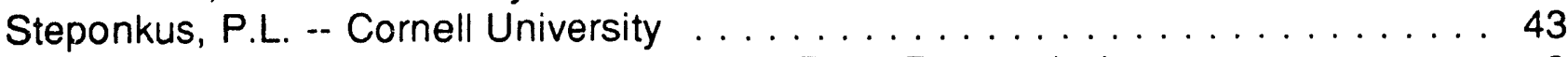

Stern, D.B. -- Boyce Thompson Institute for Plant Research, Inc. . . . . . . . . . . 9

Stewart, V.J. -- Cornell University . . . . . . . . . . . . . . . . . . . . . . . . 44

Sussman, M.R. -- University of Wisconsin . . . . . . . . . . . . . . . . . . 179

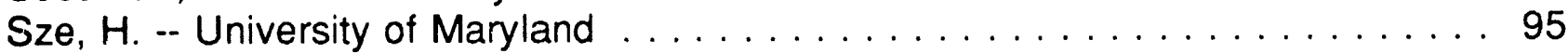

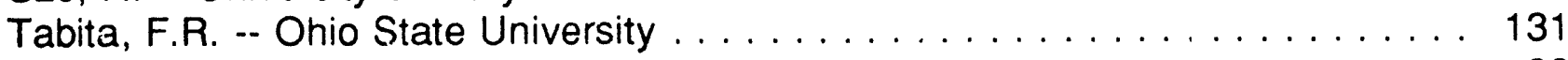

Taiz, L. -- University of California . . . . . . . . . . . . . . . . . . . 33

Thompson, D.H. -- Oregon Graduate Institute of Science and Technology . . . . 136

Tien, M. .- Pennsylvania State University . . . . . . . . . . . . . . . . . . . . . 142

Unkefer, C.J. -- Los Alamos National Laboratory . . . . . . . . . . . . . . . . . . . 93

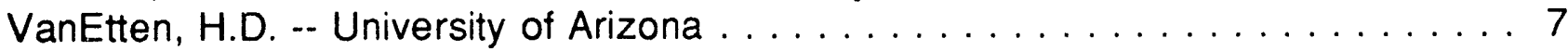

Varner, J.E. -- Washington University . . . . . . . . . . . . . . . . . . . . . . . . 169

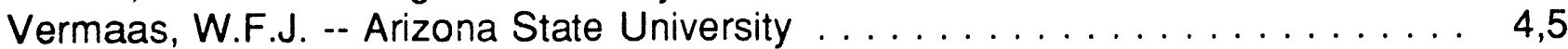

Vierstra, R.D. .- University of Wisconsin . . . . . . . . . . . . . . . . . . . 180

Wall, J.D. -- University of Missouri . . . . . . . . . . . . . . . . . . . . . . 122

Walton, J.D. -- Michigan State University DOE Plant Research Laboratory . . . . 112

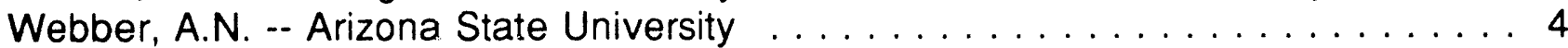

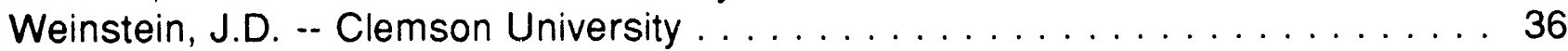

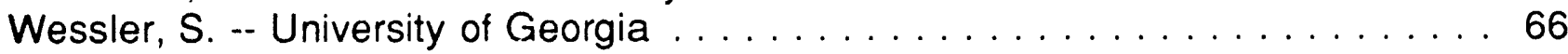

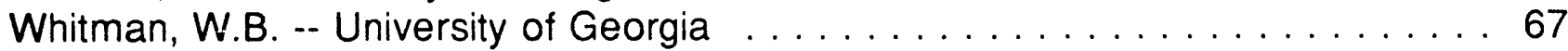

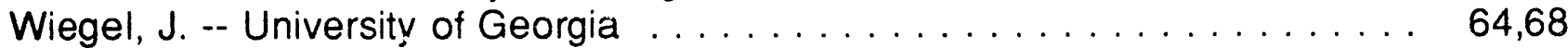

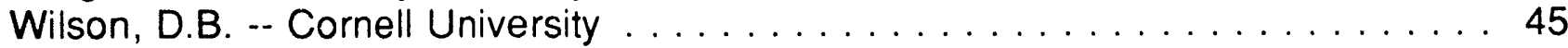

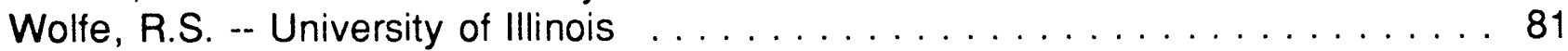

Wolk, C.P. -- Michigan State University DOE Plant Research Laboratory . . . . . 113

Wong, C.-H. -- Lawrence Berkeley Laboratory . . . . . . . . . . . . . . . . 86

Woodbury, N.W. -- Arizona State University . . . . . . . . . . . . . . . 4

Youvan, D.C. -- Massachusetts Institute of Technology . . . . . . . . . . . . 96

Zambryski, P. .- University of California . . . . . . . . . . . . . . . 23

Zeevaart, J.A.D. -- Michigan State University DOE Plant Research Lab. . . . . . . 114

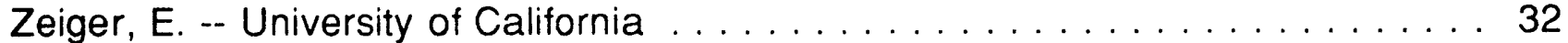

Zeikus, J.G. -- Michigan Biotechnology Institute . . . . . . . . . . . . . 100

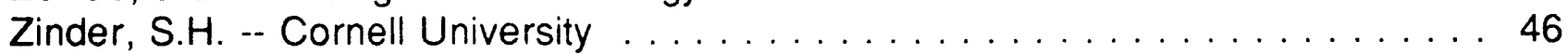




\section{SUBJECT INDEX}

ALGAE $10,12,49,65,75,92,94,131$

BACTERIA 57

ACETOGENIC 100,126

AEROBIC 20, 22, 23, 29, 44, 50, 54, 72, 77, 87, 98, 99, 111, 123, 156, 166, 167, 177, 181

ANAEROBIC $38,52,64,68,73,97,118,119,122,124$

CELLUULOLYTIC $45,73,97,150$

HALOPHILIC 27, 127

LIGNOLYTIC 71,82

METHANOGENIC $6,8,30,46,67,79,81,85,128,130,163$

METHYLOTROPHIC $16,93,120$

NITROGEN-FIXING 34, 47, 64, 97, 101, 113, 115, 137, 157, 171, 184

PHOTOSYNTHETIC $3,4,5,14,15,34,38,70,76,82,87,88,90,96,107,113,131,144,148,175,186$

SYNTROPHIC 33,133

THERMOPHIL.IC $30,46,54,55,64,68,83,127$

BIOENERGETICS (SEE TRANSPORT, PHOTOSYNTHESIS) 123

ATPASES 27, 29, 33, 65, 74, 75, 76, 79, 94, 95, 123, 145, 147, 179, 185

ELECTRON TRANSPORT $55,65,77,83,99,100,107,123,130,144,147,151$

OX. PHOSPHORYLATION 29, 50, 107, 123, 144, 147, 151, 179

PROTON TRANSPORT $29,50,55,76,77,144,145,147,179,185$

\section{CARBON METABOLISM}

CARBOHYDRATES $1,2,6,28,32,45,52,56,57,58,59,68,73,86,102,106,115,119,124,132,146$, $150,158,160,167,176,177,178,183$

CELLULOSE DEGRADATION $45,64,73,97,135,150$

HEMICELLULOSE DEGRADATION $6,68,146$

INTERMEDIARY METABOLISM 2, 10, 15, 32, 36, 44, 49, 51, 52, 53, 54, 55, 67, 68, 73, 79, 93, 100, 102, $105,110,116,119,126,133,149,150,156,162,166,167,176,178,183,186$

LIGNIN DEGRADATION $38,65,71,82,117,124,134$

ONE/TWO CARBON COMPOUNDS $8,16,30,46,52,64,67,68,79,81,85,93,100,118,120,124,126$, $128,131,133,135,156,162,163,175$

SECONDARY PRODUCTS $7,8,30,31,62,66,87,93,104,105,112,114,164,174,186$ 


\section{DEVELOPMENT}

PHOTOMORPHOGENESIS $21,89,9 \pi, 108,114,149,153,180$

PLANT $1,6,17,35,39,40,41,62,78,129,140,141,149,165,168,169$

\section{ENZYMES (SEE PROTEINS)}

MECHANISM 2, 7, 15, 29, 31, 33, 36, 38, 45, 46, 49, 52, 54, 55, 62, 64, 67, 68, 71, 74, 76, 77, 81, 83, 85, $86,87,95,96,97,99,100,102,104,105,114,116,117,126,134,137,142,145,147,150,158,160,161$, $162,163,164,166,167,170,171,172,175,176,179,186$

REGULATION 2, 6, 7, 10, 12, 13, 15, 16, 30, 33, 38, 41, 52, 53, 54, 62, 64, 64, 65, 67, 68, 71, 72, 87, 92, $100,103,105,107,109,116,117,119,120,124,131,134,142,156,160,162,163,164,167,168,170,172$, $173,175,176,177,178,186$

SECRETION $71,109,117,162,168,172,173$

\section{FERMENTATIONS}

ALGAL 10

BACTERIAL 46, 52, 64, 67, 68, 73, 81, 85, 97, 118, 119, 124, 126, 128, 133, 150, 156, 162

FUNGI $135,160,179,185$

CELLULOLYTIC 135

LIGNOLYTIC $117,134,142,172$

PATHOGENIC $7,26,56,111,112$

GENETICS (SEE MOLECULAR BIOLOGY)

CHROMOSOMAL ORGANIZATION $11,17,22,24,25,34,37,40,41,66,69,80,82,98,110,121,139,172$, $174,176,182,184$

PHENOTYPIC EXPRESSION $11,15,17,20,24,25,27,28,37,40,41,61,66,70,80,108,110,112,114$, $121,122,129,135,139,144,153,174,176$

BIOCHEMICAL GENETICS $5,11,13,15,16,17,18,20,24,25,26,27,28,31,34,37,40,41,56,61,67$, $70,75,80,87,96,105,107,108,110,111,112,114,116,117,121,122,129,135,139,144,148,150,153$, 160,162

HYDROGEN METABOLISM $10,55,64,81,83,122,133,137$

INFORMATION DATA BASE 58

MEMBRANES (SEE BIOENERGETICS, TRANSPORT, PHOTOSYNTHESIS)

PROPERTIES $43,50,110,136,181,185$

STRUCTURE $43,94,110,116,136,181$

MICROBE-MICROBE INTERACTIONS

HYDROGEN TRANSFER 46,133 
104 / Summaries of FY 1991 Activities - Subject Index

MICROBIAL ECOLOGY $46,97,133$

MOLECULAR BIOLOGY (SEE GENETICS)

NUCLEIC ACID SEQUENCE $5,7,11,12,13,14,15,16,17,18,19,20,21,22,23,24,26,27,33,34,37$, $39,41,42,44,47,48,49,52,53,60,62,63,64,65,66,67,70,71,72,73,75,77,78,79,82,83,87,92$, $96,98,100,101,103,105,107,109,110,111,112,113,116,117,119,120,121,122,123,124,125,127$, $129,130,131,134,135,137,138,139,142,143,144,148,149,150,152,153,154,156,157,158,159,160$, $162,163,164,166,167,168,170,172,173,175,176,177,178,179,180,181,182,183,184,186$

ORGANELLES $9,12,13,19,33,39,49,53,63,65,80,92,107,109,121,121,139,143,153,159,173$

PLASMIDS $15,23,42,49,67,72,73,79,113,125,127,130,134,139$

PROMOTERS AND CONTROLLING REGIONS $6,9,11,12,15,16,17,18,19,20,21,22,26,30,34,37$, $39,44,45,47,52,53,61,63,66,72,7$ \&, 79, 82, 83, 87, 101, 103, 107, 109, 112, 113, 113, 117, 119, 120, $121,122,124,127,130,131,134,135,143,148,149,152,154,156,157,159,162,163,164,167,170,172$. $177,179,182,183,184,186$

TRANSCRIPTION $9,11,12,16,18,19,21,22,23,30,31,34,37,44,47,48,53,61,63,66,78,79,82,103$, $109,113,113,120,124,125,127,129,130,135,138,139,143,148,149,152,154,157,159,164,169,183$, 184,186

TRANSLATION $9,11,12,16,18,19,21,22,23,30,31,34,37,44,47,53,61,63,66,78,79,82,92,103$, $109,113,120,124,125,130,135,138,139,143,148,152,154,157,159,164,169,173,183,184,186$

VECTORS $9,14,16,19,23,26,28,37,45,52,60,66,67,73,79,98,113,113,119,122,124,127,130$, $134,152,154,172,180$

\section{NITROGEN METABOLISM}

NITROGEN FIXATION $34,47,64,97,101,115,137,157,171$

NODULE DEVELOPMENT $47,64,101,115,157,171$

NUTRITION $34,44,65,97,105,171$

\section{PHOTOSYNTHESIS}

CARBON FIXATION $10,32,49,51,63,75,131,178,183$

ELECTRON FLOW $3,4,5,13,75,84,88,90,94,107,144,148,161$

OXYGEN EVOLUTION $10,88,90,148,155$

MEMBRANE ASSEMBLY AND FUNCTION 5, 13, 70, 84, 94, 107, 143, 148, 155, 161, 173

PHOTOPHOSPHORYLATION $65,75,76,90,96,144,147$

PHOTOREACTIONS $3,4,5,13,14,27,75,84,88,90,94,96,107,155$

REACTION CENTERS $3,4,5,12,13,14,15,27,70,84,88,90,94,96,107,143,148,161$

\section{PLANT PHYSIOLOGY}

GROWTH REGULATORS AND FLOWERING $17,21,31,51,56,60,62,78,91,105,108,114,129,140$, $154,168,170$ 
WATER RELATIONS $32,48,51,60,104,140$

PLANT

ASSIMILATE PARTITIONING $2,149,178$

DICOT $1,2,6,7,9,13,17,19,20,21,22,23,24,26,28,29,33,35,36,39,40,41,42,47,48,51,53,56$, $57,59,60,61,62,63,74,78,84,88,91,95,101,102,103,104,105,106,107,108,109,110,111,114,116$, $125,129,132,138,140,141,143,145,147,149,151,153,154,155,157,158,161,164,166,167,168,169$, $170,171,173,177,178,179,180,183,185$

GYMNOSPERM $1,106,165$

MONOCOT $11,18,21,25,31,32,37,43,50,57,62,66,69,80,106,107,111,112,121,139,146,152$, $159,168,174,176,180,182$

\section{PLANT-MICROBIAL INTERACTIONS}

MICROBIAL PATHOGENS 7, 20, 22, 23, 26, 50, 56, 91, 111, 112, 177

NITROGEN SYMBIOSIS $47,101,115,157,171$

PROTEINS (SEE ENZYMES)

STRESS $60,72,76$

PROTEASES $45,138,168,180$

STRUCTURAL SUPPORT $86,106,152,1 \div 4,169,181$

STRUCTURE $2,5,13,21,28,29,31,36,38,42,45,46,50,52,53,53,60,62,64,68,74,76,77,78,81$, $83,85,86,87,89,90,94,95,96,97,99,100,102,104,105,106,107,109,110,112,114,116,117,120$, $123,126,128,131,135,137,141,144,145,146,147,155,156,158,160,161,162,163,164,166,167,170$, $171,173,175,176,179,180,183$

\section{STRESS}

CHEMICAL TOXICITY 72,118

PATHOGEN 20, 24, 35, 42, 50, 56, 91, 111, 112, 125, 138, 165, 177

SALINITY $8,25,30,33,98,104,127$

TEMPERATURE $20,43,54,61,98,114,134,151$

WATER $32,48,51,60,104,140$

$\mathrm{PH} 98,123$

CHEMICAL STRUCTURE (SEE MOLECULAR BIOLOGY, PROTEINS)

CELL WALL $1,56,57,59,102,106,115,129,132,140,146,158,160,165,169$

LIPIDS $43,110,116$

SULFUR METABOLISM

REDUCTION 83, 122 
106 / Summaries of FY 1991 Activities - Subject Index

TECHNIQUES - SPECIALIZED

EXAFS, EPR, NMR $1,4,8,55,56,57,59,64,77,84,93,115,126,132,136,137,142,155,161,165,175$ I.IGHT SPECTROSCOPY-SPECIALIZED $3,4,14,27,84,88,90$

TRANSPORT (SEE BIOENERGETICS)

ION $25,33,60,74,76,95,141,145,147$

INORGANIC COMPOUNDS 27

ORGANIC COMPOUNDS 32

VIRUS $14,24,42,125,138$ 

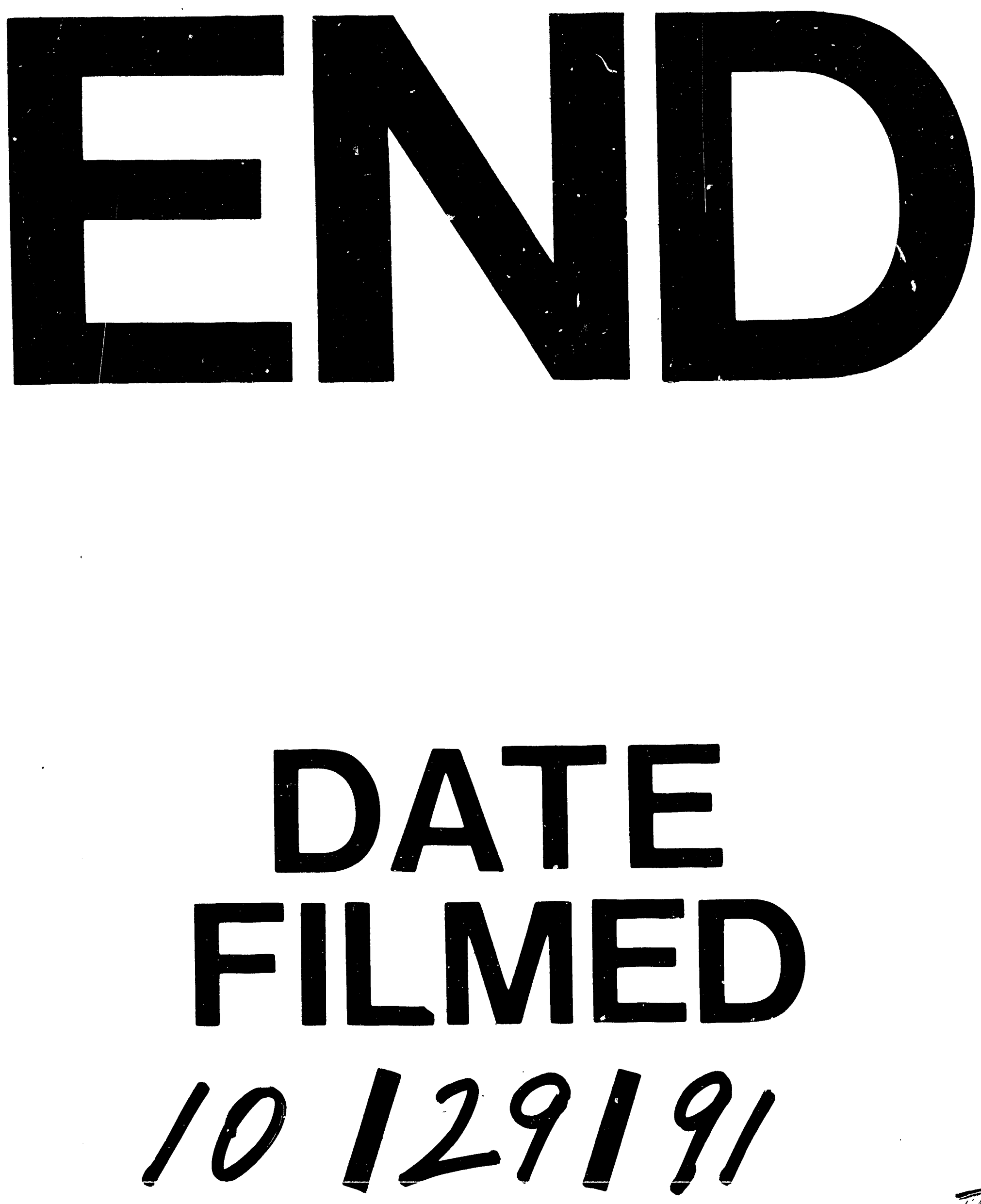

II 
